\title{
A joint location inventory model under the risk of supply disruptions with facility fortifications
}

\author{
Ehsan Jafari Shirazi
}

Follow this and additional works at: https://researchrepository.wvu.edu/etd

\section{Recommended Citation}

Shirazi, Ehsan Jafari, "A joint location inventory model under the risk of supply disruptions with facility fortifications" (2015). Graduate Theses, Dissertations, and Problem Reports. 7346.

https://researchrepository.wvu.edu/etd/7346

This Dissertation is protected by copyright and/or related rights. It has been brought to you by the The Research Repository @ WVU with permission from the rights-holder(s). You are free to use this Dissertation in any way that is permitted by the copyright and related rights legislation that applies to your use. For other uses you must obtain permission from the rights-holder(s) directly, unless additional rights are indicated by a Creative Commons license in the record and/ or on the work itself. This Dissertation has been accepted for inclusion in WVU Graduate Theses, Dissertations, and Problem Reports collection by an authorized administrator of The Research Repository @ WVU.

For more information, please contact researchrepository@mail.wvu.edu. 


\title{
A joint location inventory model under the risk of supply disruptions with facility fortifications
}

\author{
Ehsan Jafari Shirazi \\ Dissertation Submitted to the \\ College of Engineering and Mineral Resources \\ at West Virginia University \\ in partial fulfillment of the requirements for the degree of \\ Doctor of Philosophy in \\ Industrial Engineering \\ Wafik Iskander, Ph.D., Chair \\ Majid Jaridi, Ph.D. \\ Feng Yang, Ph.D. \\ Powsiri Klinkhachorn, Ph.D. \\ Bhaskaran Gopalakrishnan, Ph.D.
}

Department of Industrial and Management Systems Engineering

Morgantown, West Virginia 2015

Keywords: Reliability fixed-charge location problem, Joint location inventory problem, Facility fortifications, Global search techniques

Copyright 2015 Ehsan Jafari Shirazi 
ProQuest Number: 3741894

All rights reserved

INFORMATION TO ALL USERS

The quality of this reproduction is dependent upon the quality of the copy submitted.

In the unlikely event that the author did not send a complete manuscript and there are missing pages, these will be noted. Also, if material had to be removed, a note will indicate the deletion.

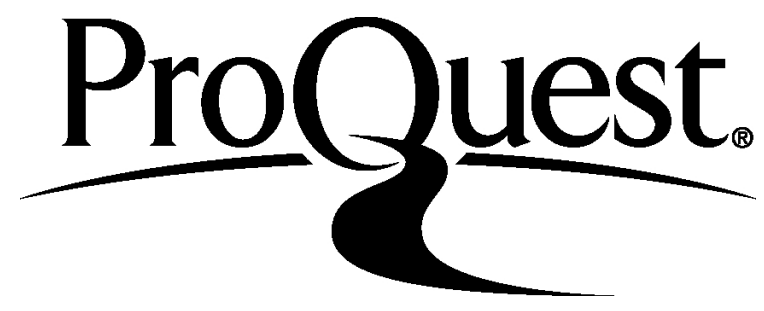

ProQuest 3741894

Published by ProQuest LLC (2015). Copyright of the Dissertation is held by the Author.

All rights reserved.

This work is protected against unauthorized copying under Title 17, United States Code Microform Edition (c) ProQuest LLC.

ProQuest LLC.

789 East Eisenhower Parkway

P.O. Box 1346

Ann Arbor, MI $48106-1346$ 


\section{Abstract \\ A joint location inventory model under the risk of supply disruptions with facility fortifications \\ Ehsan Jafari Shirazi}

Inefficient facility location decisions result in extra cost throughout the lifetime of the facilities. Hence, facility location decisions are considered as critical and difficult decisions in today's highly competitive business environment. There are two relatively new areas of research in facility location modeling approaches that have recently received much attention. The first is the incorporation of short-term inventory decisions into long-term location decisions. It has been shown in the literature (e.g. Daskin et al. 2002 or Shen et al. 2003) that failing to account for inventory may result in fundamental changes in the location decisions and thus suboptimality. The second considers the relaxation of the assumption in classical facility location models that facilities are always available and perfectly reliable. In reality, facilities are subject to disruptions due to different unexpected events such as natural disasters or terrorism. The interest in this avenue of research has been increasing in the past few years, triggered by recent high-profile disastrous events such as those of September 11, 2001 and hurricane Katrina in 2005. While the importance of the integration of the two aforementioned areas of research has been clarified in the literature, the integration studies are scarce due to the difficulty and challenges that are presented by the incorporation of the nonlinear inventory costs into a reliability location framework. This dissertation integrates the two areas of research and proposes a location-inventory model that also considers facility fortification as a proactive strategy to hedge against disruptions. Two global search methodologies are developed to solve the problem, and their computational efficiencies are investigated under different parameter settings. 


\section{Dedication}

Dedicated to the loving memory of my grandmother... 


\section{Acknowledgements}

I would like to especially thank my advisor, Dr. Wafik Iskander, for all the time that he spent on every single page of this dissertation to ensure the quality of the research, and for constantly providing me with invaluable comments and insights during the completion of this dissertation. I also greatly appreciate his guidance, teaching, great mentoring and kind support throughout my Ph.D. studies.

I would like to thank Dr. Majid Jaridi for his valuable comments and suggestions, and for all of his support and encouragement during my $\mathrm{Ph} . \mathrm{D}$. studies.

I am also thankful to Dr. Feng Yang, Dr. Powsiri Klinkhachorn and Dr. Bhaskaran Gopalakrishnan for their great ideas and feedbacks through this process.

Last but not the lease, I would like to thank my family, for always being there to support me. 


\section{TABLE OF CONTENTS}

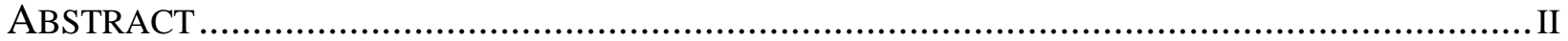

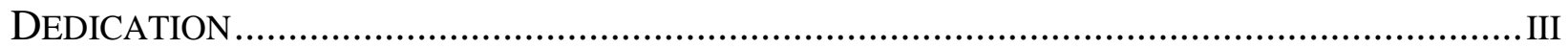

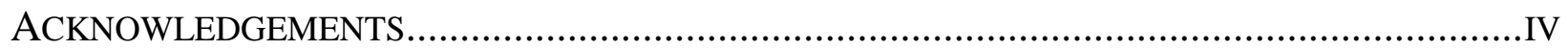

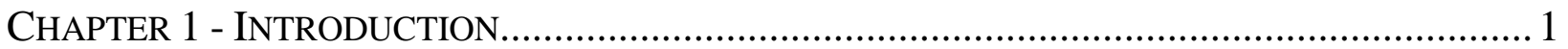

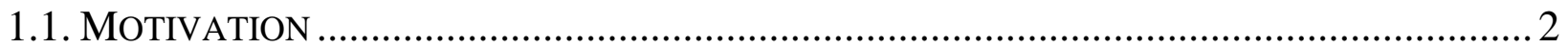

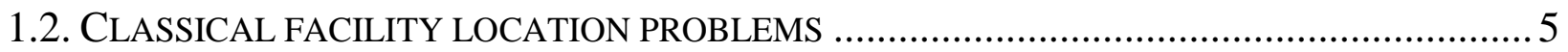

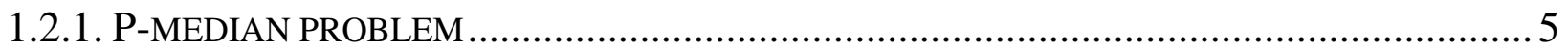

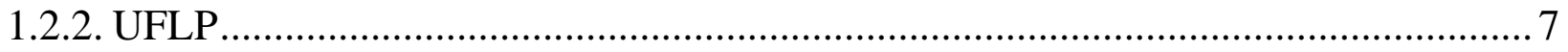

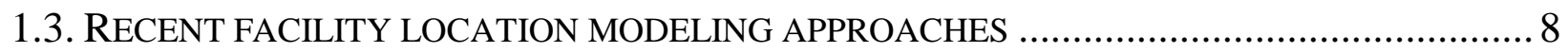

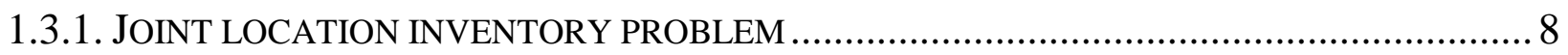

1.3.2. RELIABILITY FIXED-CHARGE LOCATION PROBLEM (RFLP) …............................... 11

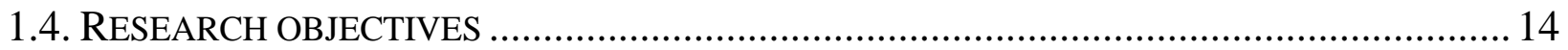

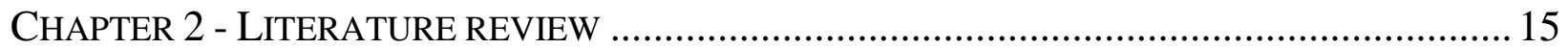

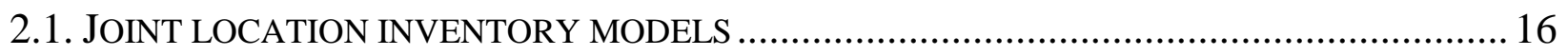

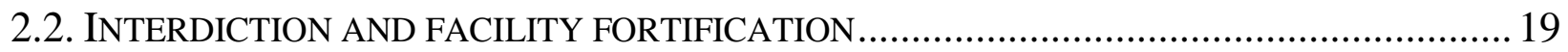

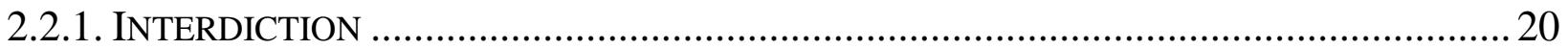

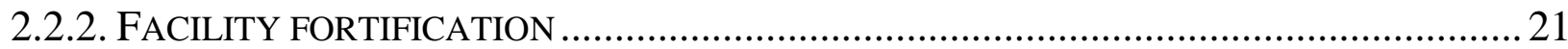


2.3. FACILITY DISRUPTION MODELS

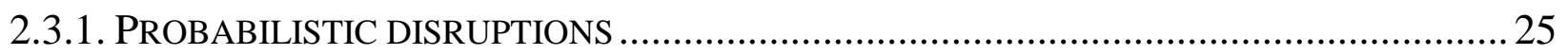

2.3.2. RANDOM DISRUPTION MODELS WITH FACILITY FORTIFICATIONS ............................ 30

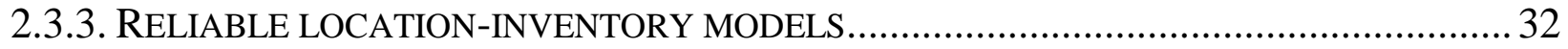

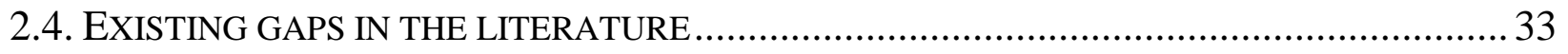

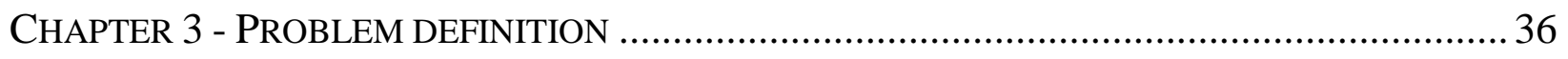

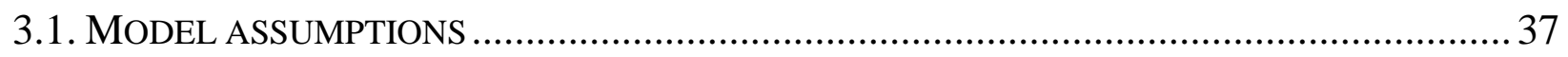

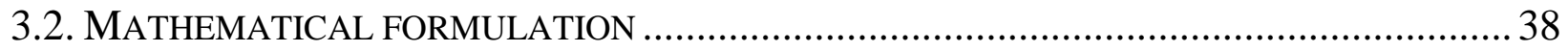

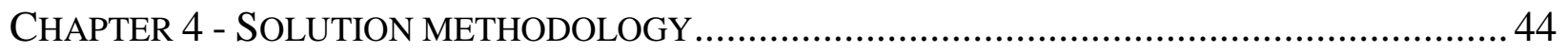

4.1. REFORMULATION OF THE PROPOSED MODEL …..................................................... 45

4.2. CONVEXITY PROOF FOR THE NONLINEAR TERMS ……........................................... 49

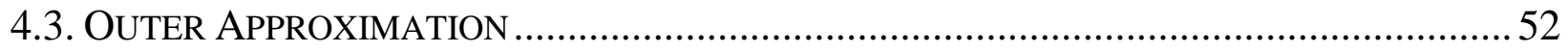

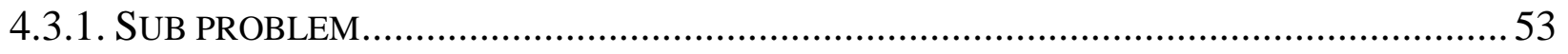

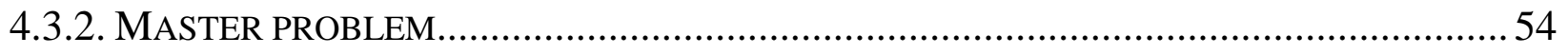

4.4. LP/NLP BASED BRANCH AND BOUND FRAMEWORK ..............................................59

4.4.1. IMPLEMENTATION OF LP/NLP-BB FOR PROBLEM (P2) ........................................ 60

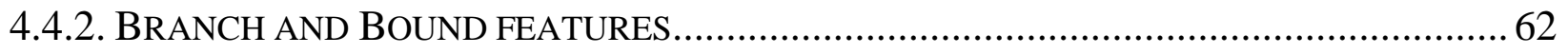

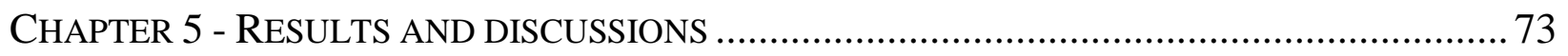




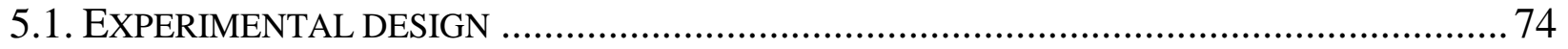

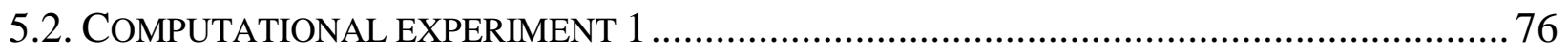

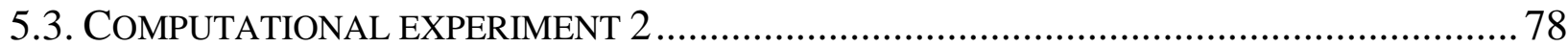

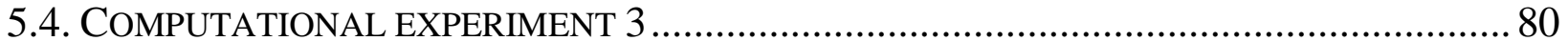

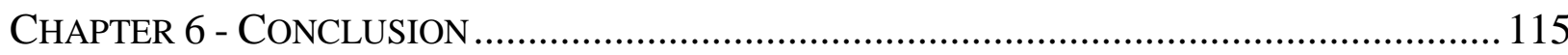

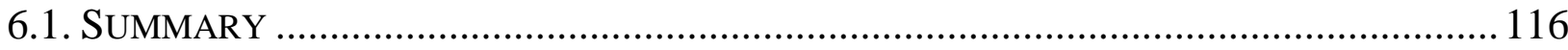

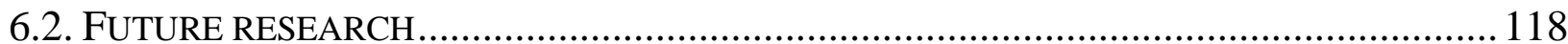

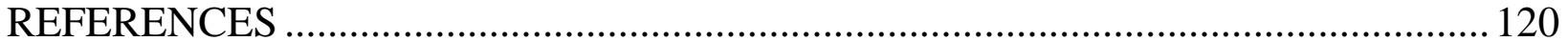




\section{LIST OF TABLES}

TABLE 2.1.EXISTING GAPS IN THE LITERATURE THAT IS TO BE COVERED BY THIS

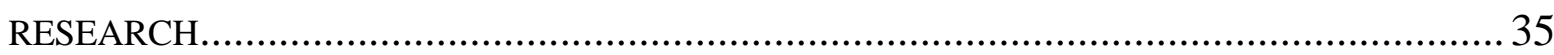

TABLE 4.1. OUTER APPROXIMATION (OA) ALGORITHM STEPS .........................................58

TABLE 4.2. LP/NLP BASED BRANCH AND BOUND (LP/NLP-BB) ALGORITHM................... 63

TABLE 4.3. RELAXATION INDUCED NEIGHBORHOOD SEARCH (RINS) WITHIN LP/NLP-BB

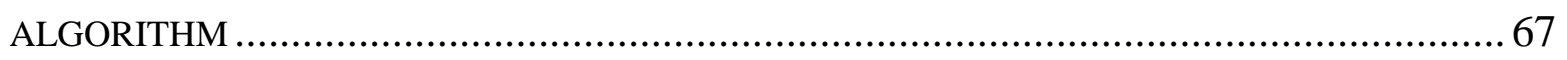

TABLE 4.4. LINEARIZATION GENERATION AT FRACTIONAL SOLUTIONS FOR THE LPR-MP

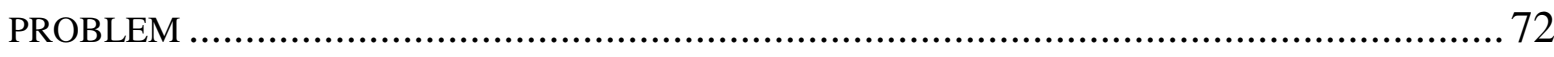

TABLE 5.1. BASE VALUES FOR MODEL PARAMETERS.................................................... 76

TABLE 5.2. COMPARISONS OF BARON SOLVER WITH OA AND LP/NLP-BB ALGORITHMS

TABLE 5.3. COMPARISONS OF OA AND LP/NLP-BB ALGORITHMS WITH $\mathrm{B}=0 \ldots \ldots \ldots \ldots \ldots \ldots . . . .82$

TABLE 5.4. COMPARISONS OF OA AND LP/NLP-BB ALGORITHMS WITH $B=30 \ldots \ldots \ldots \ldots \ldots . . .83$

TABLE 5.5. COMPARISONS OF OA AND LP/NLP-BB ALGORITHMS WITH B $=60 \ldots \ldots \ldots \ldots \ldots . . . .84$

TABLE 5.6. COMPARISONS OF OA AND LP/NLP-BB ALGORITHMS WITH B=120 .............. 85

TABLE 5.7. COMPARISONS OF OA AND LP/NLP-BB ALGORITHMS WITH $B=180 \ldots \ldots \ldots \ldots . . . .86$

TABLE 5.8. COMPARISONS OF OA AND LP/NLP-BB ALGORITHMS WITH $B=240 \ldots \ldots \ldots \ldots . . . .87$

TABLE 5.9. COMPARISONS OF OA AND LP/NLP-BB ALGORITHMS WITH $B=300 \ldots \ldots \ldots \ldots . . . .88$ viii 
TABLE 5.10. COMPARISONS OF OA AND LP/NLP-BB ALGORITHMS WITH B=360. 89

TABLE 5.11.A. LP/NLP-BB COMPUTATIONAL PERFORMANCE FOR 150-NODE PROBLEMS, $\mathrm{Q}=0.05, \mathrm{R}=3$ 90

TABLE 5.11.B. LP/NLP-BB COMPUTATIONAL PERFORMANCE FOR 150-NODE PROBLEMS, $\mathrm{Q}=0.05, \mathrm{R}=3$

TABLE 5.11.C. LP/NLP-BB COMPUTATIONAL PERFORMANCE FOR 150-NODE PROBLEMS, $\mathrm{Q}=0.05, \mathrm{R}=3$ 92

TABLE 5.11.D. LP/NLP-BB COMPUTATIONAL PERFORMANCE FOR 150-NODE PROBLEMS, $\mathrm{Q}=0.05, \mathrm{R}=3$ 93

TABLE 5.12.A. LP/NLP-BB COMPUTATIONAL PERFORMANCE FOR 150-NODE PROBLEMS, $\mathrm{Q}=0.05, \mathrm{R}=2$ .94

TABLE 5.12.B. LP/NLP-BB COMPUTATIONAL PERFORMANCE FOR 150-NODE PROBLEMS, $\mathrm{Q}=0.05, \mathrm{R}=2$ 95

TABLE 5.12.C. LP/NLP-BB COMPUTATIONAL PERFORMANCE FOR 150-NODE PROBLEMS, $\mathrm{Q}=0.05, \mathrm{R}=2$ 96

TABLE 5.12.D. LP/NLP-BB COMPUTATIONAL PERFORMANCE FOR 150-NODE PROBLEMS, $\mathrm{Q}=0.05, \mathrm{R}=2$ 97

TABLE 5.13.A. LP/NLP-BB COMPUTATIONAL PERFORMANCE FOR 150-NODE PROBLEMS, $\mathrm{Q}=0.05, \mathrm{R}=4$ 98 
TABLE 5.13.B. LP/NLP-BB COMPUTATIONAL PERFORMANCE FOR 150-NODE PROBLEMS, $\mathrm{Q}=0.05, \mathrm{R}=4$ 99

TABLE 5.13.C. LP/NLP-BB COMPUTATIONAL PERFORMANCE FOR 150-NODE PROBLEMS, $\mathrm{Q}=0.05, \mathrm{R}=4$ 100

TABLE 5.13.D. LP/NLP-BB COMPUTATIONAL PERFORMANCE FOR 150-NODE PROBLEMS, $\mathrm{Q}=0.05, \mathrm{R}=4$ 101

TABLE 5.14.A. LP/NLP-BB COMPUTATIONAL PERFORMANCE FOR 150-NODE PROBLEMS, $\mathrm{Q}=0.05, \mathrm{R}=5$ 102

TABLE 5.14.B. LP/NLP-BB COMPUTATIONAL PERFORMANCE FOR 150-NODE PROBLEMS, $\mathrm{Q}=0.05, \mathrm{R}=5$ 103

TABLE 5.14.C. LP/NLP-BB COMPUTATIONAL PERFORMANCE FOR 150-NODE PROBLEMS, $\mathrm{Q}=0.05, \mathrm{R}=5$ 104

TABLE 5.14.D. LP/NLP-BB COMPUTATIONAL PERFORMANCE FOR 150-NODE PROBLEMS, $\mathrm{Q}=0.05, \mathrm{R}=5$ 105

TABLE 5.15.A. LP/NLP-BB COMPUTATIONAL PERFORMANCE FOR 150-NODE PROBLEMS, $\mathrm{Q}=0.01, \mathrm{R}=3$ 106

TABLE 5.15.B. LP/NLP-BB COMPUTATIONAL PERFORMANCE FOR 150-NODE PROBLEMS, $\mathrm{Q}=0.01, \mathrm{R}=3$ 107

TABLE 5.15.C. LP/NLP-BB COMPUTATIONAL PERFORMANCE FOR 150-NODE PROBLEMS, $\mathrm{Q}=0.01, \mathrm{R}=3$ 108 
TABLE 5.15.D. LP/NLP-BB COMPUTATIONAL PERFORMANCE FOR 150-NODE PROBLEMS,

$\mathrm{Q}=0.01, \mathrm{R}=3$

TABLE 5.16.A. LP/NLP-BB COMPUTATIONAL PERFORMANCE FOR 150-NODE PROBLEMS, $\mathrm{Q}=0.03, \mathrm{R}=3$ 110

TABLE 5.16.B. LP/NLP-BB COMPUTATIONAL PERFORMANCE FOR 150-NODE PROBLEMS, $\mathrm{Q}=0.03, \mathrm{R}=3$ 111

TABLE 5.16.C. LP/NLP-BB COMPUTATIONAL PERFORMANCE FOR 150-NODE PROBLEMS, $\mathrm{Q}=0.03, \mathrm{R}=3$

TABLE 5.16.D. LP/NLP-BB COMPUTATIONAL PERFORMANCE FOR 150-NODE PROBLEMS, $\mathrm{Q}=0.03, \mathrm{R}=3$ 113

TABLE 5.17. ADVANTAGE OF PLANNING FOR DISRUPTION: AN EXAMPLE FOR 150-NODE PROBLEMS, $\mathrm{Q}=0.001, \mathrm{R}=3, \mathrm{~B}=180$ 114 


$\begin{array}{ll}\text { BARON } & \text { Branch-And-Reduce Optimization Navigator } \\ \text { BB } & \text { Branch and Bound } \\ \text { ECP } & \text { Extended Cutting Plane Method } \\ \text { EOQ } & \text { Economic Order Quantity } \\ \text { GAMS } & \text { General Algebraic Modeling System } \\ \text { GBD } & \text { Generalized Benders Decomposition } \\ \text { LPR-MP } & \text { LP relaxation master problem } \\ \text { LP/NLP-BB } & \text { LP/NLP based branch and bound } \\ \text { MILP } & \text { Mixed Integer Linear Program } \\ \text { MINLP } & \text { Mixed Integer Nonlinear Program } \\ \text { Mii } & \text { Master Problem } \\ \text { RFLP } & \text { Non-Linear Program } \\ \text { NLP } & \text { Outer Approximation } \\ \text { OA } & \text { Relaxation Induced Neighborhood Search } \\ & \end{array}$




\section{Chapter 1 - Introduction}




\subsection{Motivation}

Traditional facility location models locate facilities ${ }^{1}$, given a set of candidate locations, and assign a set of customers to each of the located facilities with the objective of minimizing the total cost, which is the summation of a fixed facility setup cost and a variable transportation cost that is proportional to the distance between customers and facilities. The facility location problem is actually a part of a bigger problem, the Supply Chain Management (SCM). The SCM not only includes decisions of locating plants or distribution centers, but also decisions about production plans, routing and transportation options, inventory management, and information sharing throughout the whole chain.

While production quantities, routing, inventory, and information management decisions are relatively flexible for short term or middle term changes in the underlying conditions of the supply chain, facility location decisions are often fixed and difficult to change even in the intermediate term. In other words, facility location decisions are specified as strategic whereas the other decisions are considered as tactical or operational. If the long term decisions of locating production facilities or distribution centers are made inefficiently, not only extra costs are incurred throughout the lifetime of the facilities, but also the shorter term decisions like routing or inventory are affected by the resultant inefficient configuration of the facilities. Therefore, location decisions may be seen as the key and the most critical decisions that need to be made for an efficient supply chain.

Traditional studies have treated long term location decisions in isolation and separated them from the shorter term decisions. However, more recent works in the literature suggest that the integration of these decisions provides for better optimality of the supply chain design. This is

\footnotetext{
${ }^{1}$ Facilities or distribution centers are used interchangeably throughout this dissertation.
} 
specifically true about the incorporation of inventory into facility location decisions, since it has been shown that the location decisions that are made by an approach that fails to account for inventory can be radically different from those made while considering inventory. In other words, failing to merge inventory and location decisions may result in suboptimality (Daskin et al. 2002, Shen et al. 2003). Although this fact was implicitly noticed in a much earlier study by Baumol and Wolfe (1958), the nonlinearity associated with embedding inventory decisions into location models, and the difficulty of solving the problem, had restricted further work until significant improvements in computer technologies over the past decades, coupled with developments of commercial optimization software packages like ILOG CPLEX, made it possible to tackle the problem for fairly large instances. The studies by Daskin et al. (2002) and Shen et al. (2003) opened a new avenue of research that has received considerable attention over the past few years. Nevertheless, there is a lot of room for more research to be done in this area, such as combining the idea of joint location inventory models with other recent advances in facility location modeling (like the one outlined below) to develop more integrated models.

An assumption in the classical facility location models is that facilities are always available and perfectly reliable. In reality, facilities are subject to disruptions due to different unexpected events such as natural disasters, power outages, transportation blockages, labor strikes, and terrorism. For instance, a fire event in year 2000 halted a Philips semiconductor plant in New Mexico for nine months, causing shortages of cell phone components, which resulted in losing a substantial portion of Ericsson's market to its rival, Nokia. Following September 11, 2001, all U.S. borders were closed, which forced Ford to idle several assembly lines due to the shortage of parts supplied from abroad. More recent examples include hurricane Katrina in 2005, which caused a severe disruption to the crude oil production in the Gulf of Mexico. These examples show the 
significant consequences of facility disruptions and the need to account for them in the design phase. This need is more appreciated considering two other evidences beside the high-profile disruptions in recent years. The first one is that Just-In-Time (JIT) and lean practice strive to increase efficiency and decrease waste and redundancies, which although function well under normal situations, leave no room for severe failures or disruptions. The second evidence is that in today's globalized supply chain, suppliers or sub-suppliers are located throughout the world, and in some cases in countries that are not politically or economically stable. Therefore, researchers have become increasingly interested in supply chain disruptions over the past years and studied developments of different mitigation approaches.

Most of the work in the literature has used reactive strategies to hedge against disruptions. That is the incorporation of backup facilities for disrupted or failed facilities. For instance, Snyder and Daskin (2005) pioneered two reliability models based on classical facility location models, the P-median problem and the uncapacitated fixed-charge location problem, which they called RPMP and RFLP ${ }^{1}$, respectively. In their proposed models, a customer's demand is assigned to " $\mathrm{R}$ " consecutive facilities, one primary and "R-1" levels of backup. In other words, the customer is reassigned to the next available facility whenever its assigned facility fails. In addition to reactive strategies, Lim et al. (2010), followed by Li et al. (2013), suggested facility fortifications as a proactive remedy to improve the reliability of facilities. In this case, by investing extra money, a facility can be fortified and consequently becomes non-failable.

In this dissertation, we integrate the aforementioned facility location modelling approaches and propose a reliable location-inventory model considering both reactive (back-up facilities) and proactive (facility fortifications) strategies against disruptions. In the rest of this chapter, some

\footnotetext{
${ }^{1}$ Reliability P-Median Problem and Reliability Fixed-charge Location Problem
} 
base models for the proposed problem are described in detail, followed by the objectives of the proposed study.

\subsection{Classical facility location problems}

The facility location problem has been broadly studied in the literature. This problem can be classified into two categories: deterministic and stochastic. In this section, the deterministic problems $^{1}$, P-median and Uncapacitated Fixed-charge Location Problem (UFLP), are presented. ${ }^{2}$ These problems are the basis for many other location models that have been used in the literature. The P-median problem differs from the UFLP in two respects: there is a certain number of "P" facilities that should be opened, and there are no fixed setup costs for opening these facilities. Both of these problems are described below.

\subsubsection{P-median problem}

The P-median problem is to select "P" facilities from a set of candidate locations in order to minimize the sum of the total demand-weighted distance between retailers and facilities. The Pmedian problem is useful in modeling many real world situations such as the location of public or industrial facilities, warehouses, police stations, or hospitals. The P-median problem can be formulated as follows:

\footnotetext{
${ }^{1}$ The reader may refer to Owen and Daskin (1998) or Snyder (2006) for a comprehensive review on stochastic facility location problems with uncertainty.

${ }^{2}$ We are using studies by Hakimi (1964) and Balinski (1965) for P-median and UFLP, respectively.
} 


\section{$\underline{\text { Indices }}$}

$i$

j

\section{$\underline{\text { Parameters }}$}

$P$
$c_{i j}$
$\lambda_{i}$

$\underline{\text { Decision Variables }}$

$y_{j} \in\{0,1\}$

$x_{i j} \in\{0,1\}$

P-median problem:

$\operatorname{Min} \sum_{i} \sum_{j} \lambda_{i} c_{i j} x_{i j}$

subject to:

$$
\begin{array}{ll}
\sum_{j} x_{i j}=1, & ; \text { for } i=1, \ldots, I, \\
\sum_{j} y_{j} \leq P & \\
x_{i j} \leq y_{j}, & \text {; for } i=1, \ldots, I, j \\
x_{i j}, y_{j} \in\{0,1\} & \text {; for } i=1, \ldots, I, j
\end{array}
$$

In the above formulation, the objective function (1.1) is to minimize the total demandweighted distance between retailers and facilities. Constraint (1.2) ensures that each retailer is 
assigned to only one facility (single sourcing). Constraint (1.3) enforces the number of facilities to be opened must be less than or equal to "P". Constraint (1.4) assures that a retailer can be assigned to only opened facilities. Finally constraint (1.5) illustrates the integrality constraints.

\subsubsection{UFLP}

As stated earlier, there is a predetermined number of facilities $(\mathrm{P})$ to be opened in the Pmedian problem. This is not the case for the uncapacitated fixed-charge location problem (UFLP). However, the UFLP includes one more cost in the objective function, the fixed setup cost $\left(f_{j}\right)$ for opening a facility at a candidate site $j$. Hence, the mathematical formulation of UFLP is as follows:

$\operatorname{Min} \sum_{j} f_{j} y_{j}+\sum_{i} \sum_{j} \lambda_{i} c_{i j} x_{i j}$

subject to:

$$
\begin{array}{ll}
\sum_{j} x_{i j}=1, & ; \text { for } i=1, \ldots, I \\
x_{i j} \leq y_{j}, & ; \text { for } i=1, \ldots, I, j=1, \ldots, J \\
x_{i j}, y_{j} \in\{0,1\} & ; \text { for } i=1, \ldots, I, j=1, \ldots, J
\end{array}
$$

Both the P-median and the UFLP have the following assumptions:

- There is no capacity limitation for the facilities.

- The demand is already known and deterministic.

- The facilities are always available.

- Nonlinear inventory costs are not taken into account. 


\subsection{Recent facility location modeling approaches}

As mentioned in the previous section, both P-median and UFLP problems do not take into account facility failures and inventory management decisions. Facility failures may result in excessive transportation costs since retailers may have to be served from facilities much farther than their primarily assigned facilities. In addition, incorporating inventory decisions into the facility location problem may significantly change the location decisions. In this section, two models that are considered as the basis for the model developed in this research are presented. The first one is based on the joint location inventory model studied by Daskin et al. (2002), and the second is a modified version of reliability fixed-charge location problem (RFLP) introduced by Snyder and Daskin (2005). Both of these models are built on the classical facility location model, UFLP.

\subsubsection{Joint location inventory problem}

The joint location inventory model considers inventory decision policies in a facility location context. It is assumed that retailers hold an insignificant amount of inventory, and only opened facilities order inventory from a main supplier ${ }^{1}$ using the economic order quantity (EOQ) policy. The order quantity at each facility is determined by the demand rate served by the facility, which is a function of the assignment of customers to the facility. Daskin et al. (2002) considered uncertain demand at retailers, and thus included safety stock for opened facilities. However, they further simplified the model by considering the assumption that the ratio of the variance to the mean of the demand is identical for all retailers. This assumption reduces the number of nonlinear

\footnotetext{
${ }^{1}$ The problem is not to locate this supplier but the costs of shipments from this main supplier to facilities are considered in the model.
} 
terms from two to one, which is the same as the case when demand at the retailers is assumed to be fixed. Hence, we assume fixed demands at retailers for the joint location inventory model presented here. The problem answers the following questions:

- How many facilities should be located and where to locate them?

- Which retailers should be assigned to each facility?

- How much to order and how often to reorder at each facility?

The objective is to minimize the total location, transportation and working inventory costs. To define the problem, the following notations are presented.

\section{$\underline{\text { Indices }}$}

$i$

$j$

\section{$\underline{\text { Parameters }}$}

$f_{j}$

$c_{i j}$

$d_{j}$

$g_{j}$

$A_{j}$

$h_{j}$

$\lambda_{i}$

$\beta$

$\theta$

\section{j}

Index for retailers $(1, \ldots, I)$

Index for set of candidate locations for facilities $(1, \ldots, J)$
Fixed setup cost for facility $j$, annualized over the life of the facility Per-unit transportation cost between retailer $i$ and facility $j$ Per-unit cost of shipment from the main supplier to facility $j$ Fixed cost per shipment from the main supplier to facility $j$ Fixed inventory ordering cost at facility $j$ per order Inventory holding cost per unit per year at facility $j$ Customer demand per year at retailer $i$ Weight factor associated with the transportation cost Weight factor associated with the inventory cost 


\section{$\underline{\text { Decision Variables }}$}
$y_{j} \in\{0,1\}$
Takes a value of 1 if a facility is located at $j$
$x_{i j} \in\{0,1\}$
Takes a value of 1 if retailer $i$ is assigned to facility $j$

Location-inventory model:

$\operatorname{Min} \sum_{j} f_{j} y_{j}+\sum_{i} \sum_{j} \widehat{c_{i j}} x_{i j}+\sum_{j} K_{j} \sqrt{\sum_{i} \lambda_{i} x_{i j}}$

subject to:

$$
\begin{array}{ll}
\sum_{j} x_{i j}=1, & ; \text { for } i=1, \ldots, I, \\
x_{i j} \leq y_{j}, & ; \text { for } i=1, \ldots, I, j=1, \ldots, J \\
x_{i j}, y_{j} \in\{0,1\} & ; \text { for } i=1, \ldots, I, j=1, \ldots, J
\end{array}
$$

Where $\widehat{c_{l j}}=\beta \lambda_{i}\left(c_{i j}+d_{j}\right)$ and $K_{j}=\sqrt{2 \theta h_{j}\left(A_{j}+\beta g_{j}\right)}$.

By comparing the above formulation with the UFLP presented in section (1.2.2), we can see that the constraint sets are the same, and the only difference is in the objective function terms. A perunit shipment cost from the main supplier to facilities $\left(d_{j}\right)$ is added to the second term of the objective function, and a working inventory cost is added as the third term of the objective function. Additionally, weight factors $(\beta)$ and $(\theta)$ are added to the model for simplifying the sensitivity analysis of inventory and transportation costs. To understand the working inventory cost, we should consider that the demand at each facility is a function of the customer assignments that will be made to the facility, i.e. the demand at each facility $j$ can be obtained as $\sum_{i} \lambda_{i} x_{i j}$. Then, the total inventory cost at each facility $j$ can be obtained as: 


$$
\frac{\left(A_{j}+\beta g_{j}\right) \sum_{i} \lambda_{i} x_{i j}}{Q_{j}}+\frac{h_{j} \theta Q_{j}}{2}
$$

where $Q_{j}$ is the order size at facility $j$.

Since the model uses the EOQ model, by taking the derivative of the above equation with respect to $Q_{j}$ and seting it to zero, the optimal order quantity for facility $j$ can be obtained as:

$$
\sqrt{\frac{2\left(A_{j}+\beta g_{j}\right) \sum_{i} \lambda_{i} x_{i j}}{h_{j} \theta}}
$$

And finally by replacing the above optimal order quantity in the total annual inventory cost, equation (1.14), the optimal inventory cost for facility $j$ can be given as:

$$
\sqrt{2 \theta h_{j}\left(A_{j}+\beta g_{j}\right) \sum_{i} \lambda_{i} x_{i j}}=K_{j} \sqrt{\sum_{i} \lambda_{i} x_{i j}}
$$

where $K_{j}=\sqrt{2 \theta h_{j}\left(A_{j}+\beta g_{j}\right)}$.

In fact the inventory problem is embedded into the facility location problem and once the location of facilities and the customer assignments to them are obtained, the optimal order quantity (equation 1.15) and the optimal inventory cost at each facility (equation 1.16) can be evaluated.

\subsubsection{Reliability fixed-charge location problem (RFLP)}

The main assumption in the RFLP that separates it from the classical facility location problem is that each facility may fail with a certain probability (or more accurately, parameter) " $q$ ". As Snyder and Daskin (2005) mentioned, the parameter " $q$ " is interpreted as the fraction of time the facility is nonoperational in a distant horizon, and it can be estimated using historical data. 
Furthermore, facility failures are independent and they may happen simultaneously. When a facility fails, it cannot provide any product, and the customers assigned to it must be reassigned to an un-failed/backup facility. Consequently, each customer has " $r$ " level of assignments. A "level$r$ " assignment means that there are " $r$ - 1" closest open facilities. For example, let us assume the three closest open facilities to customer $i$ are facilities 9, 5, and 3, in that order. Then, facility 9 is the level-1 facility for this customer, 5 is its level-2 facility, and 3 is its level-3 facility. Level-1 assignments are the "primary" facilities that serve the customer under normal circumstances, while level- $r$ assignments $(r>1)$ are the "backup" facilities that serve it if all closer facilities fail.

Another assumption of the model is that if customer $i$ is not served by any facility, the firm incurs a penalty cost of $\delta_{i}$ per unit of demand. The penalty $\delta_{i}$ may represent a lost-sales cost or the cost of finding an emergency source. It is incurred if all primary and backup facilities are not available, or if it is too expensive to serve a customer from its nearest remaining operating facility. One way to model this is to include a dummy emergency facility in the set of facilities. This emergency facility has no fixed cost and never fails. The transportation cost from this facility to any customer $i$ can be considered as $\delta_{i}$. In other words, assigning a customer to the emergency facility is equivalent to not assigning it at all.

Based on the above explanations, the RFLP formulation is given as follows:

\begin{tabular}{ll}
\multicolumn{1}{l}{ Indices } \\
$i$ & Index for set of retailers $(1, \ldots, I)$ \\
$j$ & Index for set of candidate locations for facilities $(1, \ldots, J)$ \\
$u$ & Dummy emergency facility \\
$r$ & Index for level of assignment $(1, \ldots,|J|)$
\end{tabular}




\section{$\underline{\text { Parameters }}$}
$f_{j}$
Annualized fixed setup cost for facility $j$
$c_{i j}$
Per-unit transportation cost between retailer $i$ and facility $j$
$\lambda_{i}$
Constant customer demand rate at retailer $i$
$q$
Fraction of time a facility is not available
$\delta_{i}$
Per unit penalty cost for not providing service to retailer $i$
Decision Variables
$y_{j} \in\{0,1\}$
Takes a value of 1 if a facility is located at $j$
$x_{i j r} \in\{0,1\}$
Takes a value of 1 if retailer $i$ is assigned to facility $j$ at level $r$.

\section{RFLP:}

Min $\quad \sum_{j} f_{j} y_{j}+\sum_{i} \sum_{r=1}^{|J|}\left[\sum_{j \in J, j \neq u} \lambda_{i} c_{i j}(1-q) q^{r-1} x_{i j r}+\right.$ $\left.\lambda_{i} \delta_{i} q^{r-1} x_{i u r}\right]$

subject to:

$$
\begin{array}{ll}
\sum_{j} x_{i j r}+\sum_{s=1}^{r} x_{i u s}=1, & ; \text { for } i=1, \ldots, I, r=1, \ldots,|J| \\
x_{i j r} \leq y_{j}, & ; \text { for } i=1, \ldots, I, j=1, \ldots, J, r=0, \ldots,|J| \\
\sum_{r=1}^{|J|} x_{i j r} \leq 1, & ; \text { for } i=1, \ldots, I, j=1, \ldots, J \\
x_{i j r}, y_{j} \in\{0,1\} & ; \text { for } i=1, \ldots, I, j=1, \ldots, J, r=0, \ldots,|J|
\end{array}
$$


The objective function (1.17) minimizes the sum of the fixed costs and the expected transportation and lost sale costs. To explain the second term, one should note that customer $i$ is assigned to facility $j$ at level $r$ if all $r-1$ closer facilities fail, which happens with probability $q^{r-1}$, and if facility $j$ itself has not failed, which happens with probability $1-q$. Furthermore, customer $i$ may be assigned to the emergency facility at any level $r$ and incurs the lost sale cost $c_{i u}=\delta_{i}$ if its $r-1$ closest facilities fail which happens with probability $q^{r-1}$.

Constraints (1.18) require each customer $i$ to be assigned to some facility at each level $r$, unless customer $i$ has been assigned to the emergency facility at some level $\mathrm{s}<\mathrm{r}$. Constraints (1.19) assure all assignments are made to opened facilities, and constraints (1.20) prevent a customer from being assigned to the same facility at more than one level. Constraints (1.21) ensure the cardinality constraints on the decision variables.

\subsection{Research objectives}

There has not been any work in the literature that integrates reliability, location, and inventory decisions with facility fortification considerations. A broad review of the related work, followed by the exiting gaps in the literature that the proposed study tries to cover, is presented in chapter 2. In general, the main objectives of this dissertation are as follows:

- To develop a model that incorporates inventory management decisions into a reliable facility location framework that considers facility fortification during the design phase as a proactive remedy as well as reactive (back-up facilities) strategies against disruptions.

- To develop two global search techniques to solve the proposed problem and compare their computational effectiveness under different parameter settings.

- To perform sensitivity analysis, and derive managerial insights. 


\section{Chapter 2 - Literature review}


In this chapter, a thorough and detailed literature review of studies related to the proposed problem is presented. Most of the related models are built on the classical facility location problems: P-median and Uncapacitated Fixed-charge Location Problem (UFLP) which were introduced by Hakimi (1964) and Balinski (1965), respectively. As mentioned in the previous chapter, the goal of this study is to integrate inventory into reliability location models, considering facility fortifications in the design phase as an option to improve the reliability of facilities. Based on this goal, the papers that were surveyed are categorized in three groups: 1- joint location inventory models, 2- interdiction and facility fortification, and 3- facility disruption models ${ }^{1}$.

\subsection{Joint location inventory models}

In this section, the integrated facility location and inventory control problem is reviewed. In the literature devoted to joint location inventory modeling, there are two types of work that incorporate inventory planning decisions in facility location framework: work that considers the capacity restrictions on facilities, and work that does not consider such a restriction (uncapacitated cases). In this section, these two types of problems are discussed.

Most of the early work in this area studied the uncapacitated version. Daskin et al. (2002) proposed a nonlinear integer programming model in which the objective is to minimize transportation, location, and nonlinear safety stock costs. A number of heuristic approaches based on Lagrangian relaxation algorithm were used to solve the problem. Shen et al. (2003) restructured the formulation of Daskin et al. as a set covering model where they efficiently solve a nonlinear

\footnotetext{
${ }^{1}$ For a broad review of supply chain disruption models which are not directly related to this research, refer to Snyder et. al. (2006) or Snyder et. al. (2012).
} 
pricing problem for two special cases: first, the variance of demand is proportional to the mean, and second, the variance of demand is zero.

Shu et al. (2005) developed a column generation approach that relaxes the two special cases of the above mentioned work to a general instance utilizing certain special structure of the pricing problem. Teo and Shu (2004) extended the above work by determining the optimal inventory policies for distribution centers and retailers using an infinite horizon two-echelon inventory cost function. The problem was formulated as a set-partitioning integer programming model, and solved using column generation.

Shu and Sun (2006) and Snyder et al. (2007) developed a scenario based on random parameters version of the models studied by Daskin et al. (2002) and Shen et al. (2003). Similar to the work by Shen et al. (2003), Snyder et al. (2007) considered the two special cases on demand patterns and used a Lagrangian relaxation algorithm to solve the problem. However, Shu and Sun (2006) reformulated the problem as a set covering model, and provided a column generation solution approach which is not restricted to the two special cases of demand scheme.

To make the model closer to reality, several other works built upon the above studies by examining capacity restriction impacts in the problem. Miranda and Garrido (2004) combined inventory control decisions with a Capacitated Facility Location problem (CFLP). Their model locates the distribution centers, assigns retailers to them, and defines the best inventory policy at each distribution center while considering capacity limitations of the distribution centers. The resulting nonlinear mixed integer problem was solved using a Lagrangian relaxation and subgradient method. 
Miranda and Garrido (2006) considered two types of capacity constraints on distribution centers. The first one limits the maximum order quantity, while the second sets a maximum inventory level for each distribution center. The latter is a chance constraint which they showed how to reformulate as a deterministic nonlinear constraint. They called the resulting model an Inventory Location Model with Inventory and Ordering Capacity (ILM-IOC) and used a Lagrangian relaxation method to solve it.

Romeijin et al. (2007) studied the capacitated version of the model proposed by Teo and Shu (2004). They formulated the problem as a set covering model and used a column generation approach to solve it.

The relation between lead time and safety stock was undertaken in a study by Sourirajan et al. (2007), where the proposed model considers the tradeoff between lead times and inventory risk-pooling benefits. While Sourirajan et al. (2007) employed a Lagrangian heuristic approach to solve the problem, Sourirajan et al. (2009) used a genetic algorithm which can also be extended to incorporate arbitrary demand variance at retailers.

The capacitated version of the models developed by Daskin et al. (2002) and Shen et al. (2003) was studied by Özsen et al. (2008), where a Lagrangian relaxation approach was used as a solution methodology.

Atamtürk et al. (2012) studied several cases of a joint inventory location problem and illustrated how to reformulate the problem as a mixed-integer conic quadratic program (MICQP). The proposed model can be solved using commercial optimization packages such as CPLEX and MOSEK, resulting in more manageable computational solution times. As they were analyzing the 
effect of tightness of distribution center capacities, they found out the capacities in the data set from Özsen et al. (2008) were mostly loose.

\subsection{Interdiction and facility fortification}

In general, there are two types of models in the literature that include disruptions: probabilistic disruption (caused by natural events) and worst-case disruption (caused by malicious agents) models. In the probabilistic ones, disruptions are considered to be exogenous, for instance, the result of weather or power failures, while the worst-case disruption models are endogenous. That is, disruptions are caused by actors that are modeled explicitly. In other words, endogenous disruptions may be influenced by the decision maker's actions, and as a result they are explicitly modeled by decision variables within the mathematical formulation, while exogenous disruptions cannot be affected by the decision maker and are modeled using stochastic processes. As facility fortification originated from studies that consider the worst-case disruption models, we review these studies in this section.

The objective of worst-case models usually is to minimize the maximum cost of facility failures in the entire network. Models considering this type of minimax approach are similar to network interdiction problems. For examples of these models, the reader can refer to Wollmer (1964), Fulkerson and Harding (1977), Golden (1978), Janjarassuk and Linderoth (2008). These problems can be considered as Stackelberg games with a leader and a follower. The leader's goal is to disrupt the network by interdicting it, which is destroying portions of it, and the follower then tries to maximize flow or minimize the shortest path through the resulting network. The network interdiction problems, like many Stackelberg games, are usually modeled using bi-level optimization, including an inner problem and an outer problem. 
It is worth noting that here we consider interdiction models in the context of facility location. There are two main differences between the models considered in this section and the network interdiction problems: 1 -in the models surveyed here, interdictions can only occur on the nodes, rather than on the arcs of the network, which is often the case of the network interdiction problems. 2- within the context of facility location, distance or travel cost from customers to their assigned facilities is considered as a measure of interest in the interdiction model, while flows or shortest paths are considered for the network interdiction problems.

First, we review the models of interdiction where the goal is not to locate facilities, as they are already built, and then we discuss the models that locate or fortify facilities to protect against future interdictions.

\subsubsection{Interdiction}

Interdiction has been defined in the literature as the intentional disruption of a network. Church et al. (2004) proposed two interdiction problems which have a close relation to two classical facility location problems: P-median and max-covering. The assumption of their model is that the set of "P" facilities has already been located and built and the decision maker chooses " $r$ " of them to interdict in order to cause as much deterioration as possible. They named the coverage-based problem as r-interdiction covering (RIC) problem, and the distance-based one as the r-interdiction median (RIM) problem. They concluded that interdiction may result in significant increases in cost, even for small " $r$ " cases.

Church and Scaparra (2007) proposed a methodology, "reliability envelope", which considers a fixed set of "p" facilities and for each $r \in\{1, \ldots p\}$, plots the best case and the worst 
case loss that may result from an $r$-facility interdiction. They defined the "reliability envelope" as the region encircled by the curve that connects the best case points and the curve that connects the worst case points. Planners can gain information required to make strategic decisions such as which facilities to protect, which to shut down if planned disruptions are required, by the shape and width of the "reliability envelope". Furthermore, the envelope provides information about how much improvement to be expected from fortification measures.

Losada et al. (2010a) worked on a finite horizon version of the RIM problem studied by Church et al. (2004) where the $r$ interdictions happen throughout the horizon, and consequently, the facilities go through a deterministic recovery time before they become available again.

Losada et al. (2012) extended the work of Church and Scaparra (2007) to include the case in which the interdictor can impact the probability of an attack's success. They assumed in their proposed model that the interdictor has the option of choosing from a set of attack levels, with different cost and probability of success, while there is a budget constraint that should be satisfied. The authors proposed three formulations in order to find a simple way for calculating the probability that a given customer is assigned to a given facility. This calculation is difficult and it is an issue for most of the models mentioned above. Losada et al. (2012) ultimately developed a network formulation in which the assignment probabilities are represented as flow variables.

\subsubsection{Facility fortification}

As mentioned in the first chapter, fortification is a proactive strategy to hedge against disruptions. More specifically, a fortified facility is assumed to be non-disruptable. To the best of our knowledge, this concept was introduced for the first time by Church and Scaparra (2007), 
where there are "p" existing facilities in the proposed model and "q" out of the "p" are selected to fortify. The objective is to minimize the increase in demand-weighted distance caused by a worstcase r-facility interdiction. They named the model as the r-interdiction median problem with fortification (RIMF), and approached it as a tri-level problem. First, the demand-weighted distance from customers to non-interdicted facilities is minimized. Then, the interdictor attempts to maximize the minimum distance through his interdictions, and finally this maximum damage is minimized by the fortifications.

Scaparra and Church (2008b) reformulated the RIMF studied by Church and Scaparra (2007) as a maximal covering problem with precedence constraints. In order to reduce the size of the problem, they used some lower and upper bounds. Scaparra and Church (2008a) developed a new solution methodology based on a tree search procedure for the same problem. Aksen et al. (2009) extended the RIMF model to have budget constraints for the fortification resources and added the assumption of having a capacity expansion cost for the reassigned customers to noninterdicted facilities.

Liberatore et al. (2011) extended the model of Church and Scaparra's (2007) and considered the $r$ number of interdicted facilities to be stochastic. They assumed that when $r$ is determined, the attacker does the worst-case " $r$ " facility interdiction. They made an example and illustrated that failing to account for the uncertainty in the number of interdicted facilities may cause substantial error. They also showed that mis-estimating the scenario probabilities of their proposed stochastic methodology would result in less error than when the stochastic nature of "r" is ignored. Liberatore and Scaparra (2011) extended this study to consider regret based objectives. They modified the problem reduction techniques to apply them to their proposed model. 
Scaparra and Church (2012) and Liberatore et al. (2012) investigated a capacitated version of the RIMF problem studied by Church and Scaparra (2007). Liberatore et al. (2012) also considered in their model partial disruptions and disruptions that affect geographic regions, rather than individual facilities. Both works first reduce the max-min part of the problem to a single-level problem, and then use the tree search algorithm proposed by Scaparra and Church (2008a) to solve the overall problem.

O'Hanley and Church (2011) proposed a location-interdiction model based on the RIC model of Church et al. (2004), following a similar approach as Church and Scaparra (2007). The key difference between their work and the study by Church and Scaparra (2007) is the decision to locate the "P" facilities, rather than taking these as inputs to the model. The objective of the model is to select "P" facilities to maximize a weighted sum of the non-disrupted demand-weighted coverage and the minimum demand-weighted coverage which takes place after a worst-case interdiction of " $r$ " facilities.

Losada et al. (2010b) added facility fortification to the study by Losada et al. (2010a) to protect against the worst-case P-median losses. Allocation of a fixed fortification budget among the $\mathrm{P}$ existing facilities is considered in the objective function. A bi-level mixed-integer program (MIP) was formulated and two decomposition based approaches were presented to solve the problem: Benders decomposition and SVI method (super valid inequalities). The two solution methodologies were compared and the Benders decomposition method tended to perform better than the SVI method for smaller problems, and worse for larger ones. Overall, the performance of the two approaches was roughly the same. 
There are two papers that considered the context of facility location with fortification for nature reserves, O’Hanley et al. (2007a) and O'Hanley et al. (2007b). Their proposed models try to find the optimum location of nature reserves which may be disrupted or destroyed by human intrusions or natural disasters. Each species, which are comparable to customers in facility location problems, is assumed to be covered by a given set of reserves. The main difference here is that the reassignment of species to backup sites following a disruption is not possible. O'Hanley et al. (2007a) proposed two models in their paper. The first one maximizes the expected coverage of species after disruptions, and the second model maximizes a weighted sum of the coverage with no disruption and the worst-case coverage level after a disruption.

O’Hanley et al. (2007b) studied models similar to the ones proposed by O'Hanley et al. (2007a) with one main difference. They considered the fact that sites not picked as reserves may still support species. In other words, they considered disruption in a reverse order. That is, while most of location-disruption models, and also O'Hanley et al. (2007a), assume some chosen locations are randomly failable, O’Hanley et al. (2007b) presumed that some non-selected sites may randomly function.

There are other works which are not in the areas of facility location, but do consider fortification. Bailey et al. (2006) incorporated design phase decisions of a network with remedial decisions for a potential adversarial situation. They called their scheme as Stochastic Programming with Adversarial Recourse (SPAR). The model determines design decisions in the first stage of a stochastic program, with an objective function that minimizes the sum of the design costs and the expected damage from an adversary which is modeled as a Markov Decision Process (MDP). The proposed scheme can also be implemented in the context of location-interdiction problems. Scaparra and Cappanera (2011) proposed a network model along with a worst-case interdiction 
that disrupts components of the network. The objective is to find the shortest path throughout the network where fortification of the nodes is the mitigation strategy against interdictions. Golany et al. (2009) studied a model with constrained fortification resources that determines the best locations to fortify where the disruption cost at each site is associated with the severity of damages. They considered two types of disruptions, probabilistic and strategic, and described the differences between the optimal fortification plans for each case.

\subsection{Facility disruption models}

As mentioned in the previous section, there are two types of disruption models considered in the literature; Probabilistic (random) and worst-case models. The latter was reviewed in the previous section, and the probabilistic one is discussed in this section. Furthermore, two subsections are included in this section to describe the closest models to the model developed in this research. The first one considers studies that investigate fortification decisions during the design phase, and the second presents the works that incorporate inventory management into facility location under probabilistic disruptions.

\subsubsection{Probabilistic disruptions}

This section provides the literature review on facility location models with random disruption of facilities. In addition to the papers discussed in here, there is a book chapter by Snyder and Daskin (2007) that describes the research in this area in more detail.

Drezner (1987), as far as we know, introduced the first study that included facility disruptions in a facility location problem. He proposed two models, and developed a neighborhood search heuristic as the solution methodology. The first model is built on the classical p-median 
problem, and takes into account the reliability of the facilities by presuming that the facilities may fail with a given probability. The second model, which is called "( $p, q)$-center problem", assumes that $\mathrm{p}$ facilities should be located such that the maximum cost associated with at most $\mathrm{q}$ facility failures is minimized. Lee (2001) introduced a continuous version of the first model presented by Drezner (1987), and developed a heuristic solution methodology based on space-filling curves.

Snyder and Daskin (2005) introduced the reliability versions of the classical uncapacitated fixed-charge location problem (UFLP) and p-median problem, and they called them reliability fixed-charge location problem (RFLP) and reliability p-median problem (RPMP). These two models have been the base of many other studies in the area of facility disruption so far. Some of the assumptions of the model proposed in this research are also based on this work. For instance, there may be up to " $\mathrm{R}$ " levels of assignment for each customer, and it is assumed that customers are served by the nearest non-failed facility. The mathematical details of the RFLP were presented in the previous chapter in section (1.3.2). The authors considered two different weighted costs in the objective function. The first one, which is called the nominal cost, does not take into account disruptions, while the second is the expected transportation cost considering disruptions (failure cost). A Lagrangian relaxation algorithm was developed to solve the problem, and it was illustrated that a significant improvement can be obtained with respect to the reliability when accounting for disruptions and spending slightly more money, versus the nominal cost obtained with the classical optimal solution. They also showed that the risk-diversification effect was present for the RFLP, as the number of opened facilities in the optimal solution was greater than that of the classical uncapacitated fixed-charge location problem (UFLP).

Bundschuh et al. (2006) differentiated between the terms "reliability" and "robustness", and studied several integer programming models for designing robust and reliable supply chains 
considering a capacitated multi-echelon network design framework. They defined the robustness as the ability of the supply chain to retain a given level of output in the presence of failure or disruption of components of the chain, and the reliability of a supply chain as the probability that all suppliers are operable within a given time horizon and environment. Peng et al. (2011) mentioned that these definitions are restrictive, and used the same definition of reliability as adopted in Snyder and Daskin (2005). They had a broader view that associates robustness to uncertainty in the data, and reliability to uncertainty in the solution. These two papers, as well as Snyder et al. (2006), are the only papers that investigated facility disruptions in the context of a network design problem.

Berman et al. (2007) proposed a model which they called the median problem with unreliable facilities (MPUF). The model is very similar to the RPMP studied by Snyder and Daskin (2005). However, some extremely nonlinear terms are used in the model to evaluate the expected travel distance. More specifically, the probability of assigning a customer to a facility in each " $r$ " level of assignments is calculated using nonlinear terms. Although the MPUF is more general than the RPMP, it is much harder to solve. While Snyder and Daskin (2005) targeted mainly the efficiency of their lagrangian relaxation algorithm in solving the RPMP, Berman et al. (2007) derived some structural results and model behavior considering disruptions. For instance, they showed that there is a co-location effect, which is having two or more facilities located in the same node, when the disruption probabilities are large.

Berman et al. (2009) presented MPUF-II which is an extension of the MPUF by adding the assumption that customers do not have information about the disrupted facilities, and they must travel from facility to facility till they find a non-failed one. They developed some heuristics for the proposed model, and investigated the additional cost resulting from the lack of disruption 
information. Furthermore, it was illustrated that the co-location effects are even more intense for this model.

Berman and Krass (2011) studied a continuous version of the MPUF and MPUF-II models where disruptions probabilities are site specific. They were able to develop a closed-form solution for the problem, and used this solution to perform an extensive sensitivity analysis for different parameters and disruption characteristics to demonstrate their effects on the optimal locations and costs.

Shen et al. (2011) extended the RFLP model proposed by Snyder and Daskin (2005) by considering site-dependent disruption probabilities. Two formulations were proposed where one uses a scenario based stochastic programming approach, and the other includes some highly nonlinear terms same as the ones in Berman et al. (2007) to calculate the expected travel costs. Although these formulations allow for statistical dependence among disruptions, the problem size grows exponentially with the number of facilities, and using an exact solution methodology may not be practical. The authors developed a heuristic based solution using a sample average approximation introduced by Kleywegt et al. (2001), followed by two greedy heuristics.

Cui et al. (2010) proposed an alternative version of the nonlinear models studied by Berman et al. (2007) and Shen et al. (2011) for the location problem with heterogeneous disruption probabilities. The nonlinear term in the Cui model consists of products of a single continuous and a single discrete decision variable rather than multiple decision variables multiplied together as in Berman et al. (2007) and Shen et al. (2011). Two solution methodologies were proposed: The first one uses the reformulation-linearization technique (RLT), introduced by Sherali and Alameddine (1992), which leads to a model that is polynomial in problem size. The second uses a continuum 
approximation (CA) methodology (Daganzo and Newell 1986) where the parameters are considered as a continuous function of the location. The CA model can be solved in closed form to help in deriving some managerial insights.

Aboolian et al. (2013) studied the same model as Cui et al. (2010), except that the number of facilities which can be assigned to a customer is not limited. They developed a solution methodology where first a relaxed version of the problem is solved to obtain a lower bound for the optimal solution. Consequently, a feasible solution is generated, and using some neighborhood search techniques, the feasible solution is improved. The last step is to add some cuts to the relaxed problem employing a cutting plane methodology to guarantee that new feasible solutions are generated in each iteration, and these steps are repeated until the desired gap is reached. This algorithm results in better solutions than that of Cui et al. (2010) with respect to both the solution gap and the computation time.

Li and Ouyang (2010) considered correlated disruptions in their proposed model. They developed some closed-form approximations for the cost in the objective function, and utilized a CA framework. However, the developed continuum approximation model performs well mainly for continuous settings rather than realistic problems in discrete settings.

Peng et al. (2011) studied a network design problem where unreliable nodes are to be located in a network, and disruptions are modeled using a scenario based approach. However, no probabilities were assumed for the scenarios, and a p-robustness approach (Kouvelis et al. 1992, Snyder and Daskin 2006) was used where the nominal cost, which is the cost when no disruptions occur, is minimized subject to a constraint that restricts the relative regret of each scenario to be 
no more than a fixed constant p. A genetic algorithm was used to solve the complicated proposed model.

Garcia-Herreros et al. (2014) developed a two-stage stochastic model that determines the location of distribution centers as well as their capacities. They formulated the problem as a mixedinteger linear program (MILP) with an objective function that includes the expected transportation, distribution center setup costs, and the expected cost of storage at distribution centers. They reformulated the problem by adding tightening disjunctive constraints, and then used a multi-cut version of Benders decomposition, with addition of pareto-optimal cuts to strengthen the master problem, to solve the problem.

\subsubsection{Random disruption models with facility fortifications}

As opposed to the studies reviewed in section (2.2.2), which assume that the fortification decisions are made for the existing facilities (the facilities are already built), the models discussed here assume that these decisions can be made during the design phase. These two approaches result in different modeling formulations. It is worth noting that the papers in this subsection consider a combination of reactive (reassignments of customers to back-up facilities) and proactive (facility fortification) strategies against facility disruptions.

Lim et al. (2010), inspired by the work of Church and Scaparra (2007), developed a model that finds where to locate facilities, as well as which facilities to fortify, in order to protect against random disruptions rather than the worst-case considered by Church and Scaparra (2007). They called the fortification planning as facility hardening, and treated the fortification cost into the objective function by using different set of variables for the reliable (fortified) and unreliable 
facilities. In their proposed model, the reliable facilities have a higher fixed cost but they are nonfailable, and the objective is to minimize the fixed cost plus the expected transportation cost.

Lim et al. (2012) considered a continuous approximation of the model introduced by Lim et al. (2010) where customers are spread uniformly in a geometric region, and the model locates both unreliable and reliable facilities to minimize the fixed cost and expected transportation cost. The main purpose of their work was to show the effects of misestimating the disruption probabilities and the correlations between them. It was numerically shown that the expected cost of underestimation in both probabilities and correlations is greater than that of overestimation.

In contrast to the study by Lim et al. (2010), Li (2011), followed by Li et al. (2013) did not consider the extra fixed cost of locating a fortified facility into the objective function, and instead they defined a fortification budget constraint, and performed a sensitivity analysis using the rate of return on fortification investments. The consideration of budget restriction for fortification investments makes their model more practical as opposed to the work by Lim et al. (2010) which has no limitation on fortification costs. Like Snyder and Daskin (2005), Lim et al. presented two models for the P-median problem (RPMP) and for a reliable uncapacitated fixed-charge location problem which they called (RUFL). They developed a Lagrangian Relaxation (LR) algorithm to solve the models. The effectiveness of the LR solution methodology was shown in terms of computational time, and compared to the solutions obtained by a myopic policy which gives priority to the most reliable facilities for fortification budget allocations without demand topology consideration.

All the three works mentioned above considered one primary facility and one level of backup for each customer. In other words, it is assumed that each customer is assigned to one 
unreliable facility (at most) and then to a fortified facility that cannot be failed. Although this approach simplifies the formulation, it may not be practical, since in reality customers whose primary facility has failed may be assigned to other non-fortified but non-disrupted facilities. This issue is resolved in this research, as we consider " $\mathrm{R}$ " levels of assignment for each customer.

\subsubsection{Reliable location-inventory models}

The works that are discussed in this section consider nonlinear inventory costs in the model and integrate inventory management into reliable facility location models. These models can be considered as merging the location-inventory models proposed by Daskin et al. (2002) (see section 2.1) with the reliability location models presented in Section (2.3.1).

Qi and Shen (2007) proposed a three-layer supply chain model in which facilities are unreliable, customer demands are stochastic and reliable delivery of products is protected by proactively assigning customers to multiple facilities (multi-sourcing). They considered a reliability coefficient for each facility and their objective is to maximize the profit rather than to minimize the cost. This can be more practical in some cases since satisfying all potential demands, which is the goal of cost minimization, may not be always optimal or feasible, especially for the customers whose primary facility is disrupted.

Qi et al. (2010) studied a model where the facilities (retailers) and the supplier can both be disrupted, and each customer is assigned to a fixed retailer. That is, reassignment of customers to other backup facilities is not a mitigation strategy here, and the inventory at facilities is used to protect customer demands when a disruption at the supplier occurs. However, there is no remedial strategy when a facility is disrupted, and the demand of a customer assigned to a failed facility is 
backlogged at a cost. It is also assumed that some customers may not be served at all when the cost of serving them is more than the lost sale penalty cost considered for each unit of unmet demand. It was numerically illustrated that significant cost savings can be achieved by considering disruptions at the supply chain design phase rather than at the operational phase.

In contrast to the above papers, Chen et al. (2011) proposed a reliable joint locationinventory model that allows reassignments of customers whose primary facilities are disrupted. They considered a sequence of a predetermined $R$ level of assignments for each customer, and developed a customized Lagrangian Relaxation (LR) method which decomposes the problem into a set of easier sub-problems to efficiently obtain near optimal solutions. They employed the data sets provided by Snyder and Daskin (2005), which have been frequently used in the literature of reliable facility location models, to evaluate the performance of the proposed solution methodology. Furthermore, they performed some sensitivity analysis on different parameters and concluded that fewer facilities are opened when the inventory cost has more weight than the transportation cost, while more facilities are opened when either the facility failure probabilities increase or the transportation cost prevails. Aryanezhad et al. (2010) developed a very similar model except that they considered random demands for customers, and they solved the problem using a genetic algorithm. In addition, Jabbarzadeh et. al. (2012) developed a scenario-based model for facility disruptions and proposed two solution methodologies based on Lagrangian Relaxation and genetic algorithm.

\subsection{Existing gaps in the literature}

In general, the following are the existing gaps in the literature that the study in this research tries to cover: 
- An extension of the work by Li et al. (2013) by considering nonlinear inventory cost in the model, or an extension of the work by Chen et al. (2011) by considering facility fortifications in the model. In other words, developing a reliable location-inventory model with facility fortifications, which has not been studied in the literature.

- The proposed model relaxes the restrictive assumption of two levels of assignment for each customer, which was used by Lim et al. (2010), Lim et al. (2012) and Li et al. (2013), to $R$ levels of assignment. Therefore, the proposed model can provide a better trade-off between facility backup (reactive) and facility fortification (proactive) strategies.

- Li et al. (2013) assumed that if a primary facility fails, the backup is always available. In reality, the backup may not always be available, as it can be a non-fortified facility which can also fail. In this research, there is no such a restriction and at each level $r$, facilities may fail.

- In the model developed in this research, if a retailer is assigned to a fortified facility at any level $r$, it will not be assigned to any other facilities at the following back-up levels. This gives an advantage in comparison with the previous work by Li et al. (2013) in which each retailer should be assigned to a primary and a back-up facility, even if the primary facility is fortified. In essence, if a retailer is assigned to a fortified facility at any level, this retailer should not be assigned to any other facility at the following backup levels, since it is assumed that a fortified facility does not fail, and there is no need for any further back-up facility assignment. 
A summary of the above points is presented in table (2.1). Also, it should be pointed out that all the works mentioned in table (2.1), and most of the studies in the area of reliability location or location inventory, use a Lagrangian Relaxation based approach which does not guarantee to produce an optimal solution. In this research, two global search approaches are developed as it will be described in chapter 4 .

Table 2.1.Existing gaps in the literature that is to be covered by this research

\begin{tabular}{|c|c|c|c|c|c|c|}
\hline $\begin{array}{l}\text { Research } \\
\text { Component }\end{array}$ & $\begin{array}{c}\text { Snyder and } \\
\text { Daskin } \\
(2005)\end{array}$ & $\begin{array}{c}\text { Aryanezhad } \\
\text { et al. } \\
\text { (2010) }\end{array}$ & $\begin{array}{c}\text { Chen et al. } \\
\text { (2011) }\end{array}$ & $\begin{array}{c}\text { Lim et al. } \\
\text { (2010) }\end{array}$ & $\begin{array}{l}\text { Li et al. } \\
\text { (2013) }\end{array}$ & $\begin{array}{l}\text { This } \\
\text { research }\end{array}$ \\
\hline $\begin{array}{l}\text { Facility } \\
\text { Fortification } \\
\text { (Nonlinear } \\
\text { component) }\end{array}$ & & & & $\checkmark$ & $\checkmark$ & $\checkmark$ \\
\hline $\begin{array}{l}\text { Inventory cost } \\
\text { (Nonlinear } \\
\text { component) }\end{array}$ & & $\checkmark$ & $\checkmark$ & & & $\checkmark$ \\
\hline $\begin{array}{l}\text { Backup } \\
\text { layers }\end{array}$ & $R$ & $R$ & $R$ & 2 & 2 & $R$ \\
\hline $\begin{array}{l}\text { Fortification } \\
\text { Budget }\end{array}$ & & & & & $\checkmark$ & $\checkmark$ \\
\hline $\begin{array}{l}\text { Random } \\
\text { demands for } \\
\text { retailers }\end{array}$ & & $\checkmark$ & & & & \\
\hline $\begin{array}{l}\text { Mathematical } \\
\text { Optimality }\end{array}$ & $\checkmark$ & & $\checkmark$ & $\checkmark$ & $\checkmark$ & $\checkmark$ \\
\hline
\end{tabular}




\section{Chapter 3 - Problem definition}


This chapter presents the mathematical formulation of the model developed in this research, for the joint facility location inventory control problem, which considers facility disruption and facility fortification. In particular, the formulation involves four different decisions in the presence of all possible disruption scenarios: 1- a facility location problem to determine the number and location of facilities, 2- the allocation of the fortification budget to the facilities, 3-an allocation problem to determine the best assignment of retailers to located facilities, and 4inventory control decisions at each located facility.

\subsection{Model assumptions}

Specifically, the following assumptions are made in the model:

- There is a fixed setup cost for opening a facility.

- A single product is considered in this work.

- Existing suppliers are considered in the model that ship the product to different facilities.

- The locations of the main suppliers are not considered in the model. However, each facility is associated with only one supplier, and the variable and fixed costs for shipments from the supplier to the facility are determined in advance.

- Facility failures are independent, with an equal parameter $q$. Our model follows the same assumption of Snyder and Daskin (2005), which defines $q$ as the fraction of time that a non-fortified facility is not available in a distant horizon.

- If a facility fails, it cannot provide any service, and its retailers are reassigned to a back-up facility.

- Each retailer can be assigned up to $\mathrm{R}$ consecutive facilities. If a customer is not served by any facility, a penalty cost is incurred for the lost demand. 
- If a facility is fortified, it becomes non-failable.

- A total budget of $B$ is available for facility fortification costs.

- An EOQ strategy is considered for inventory control at the opened facilities.

- Demand rate at retailers are fixed.

- The lead time for order delivery is negligible.

- Facilities are not subject to any capacity limitations.

\subsection{Mathematical formulation}

We use the following notations to present the mathematical formulation of the proposed model.

\section{$\underline{\text { Indices }}$}

$i$

$j$

$r$

$u$

\section{$\underline{\text { Parameters }}$}

$f_{j}$

$c_{i j}$

$A_{j}$

$h_{j}$

$d_{j}$

$g_{j}$

\section{$f_{j}$}

Index for retailers $(1, \ldots, I)$

Index for set of candidate locations for facilities $(1, \ldots, J)$

Index for backup levels $(1, \ldots, R)$

Dummy emergency facility
Fixed setup cost for facility $j$, annualized over the life of the facility

Per-unit transportation cost between retailer $i$ and facility $j$

Fixed inventory ordering cost at facility $j$ per order

Inventory holding cost per unit per year at facility $j$

Per-unit cost of shipment from the main supplier to facility $j$

Fixed cost per shipment from the main supplier to facility $j$ 


$\begin{array}{ll}\lambda_{i} & \text { Customer demand per year at retailer } i \\ \beta & \text { Weight factor associated with the transportation cost } \\ \theta & \text { Weight factor associated with the inventory cost } \\ q & \text { Fraction of time a non-fortified facility is not available } \\ \eta & \text { Penalty cost per unit of lost demand } \\ S_{j} & \text { Fixed fortification cost for facility } j \\ B & \text { Total available fortification budget } \\ \text { Variables used in the model (values obtained from the results) } \\ D_{j} & \text { Optimal expected annual demand at facility } j \\ Q_{j}^{*} & \text { Optimal order quantity at facility } j \\ C I N V_{j}^{*} & \text { Optimal expected annual inventory cost at facility } j\end{array}$

Decision Variables
$y_{j} \in\{0,1\}$
Takes value 1 if a facility is located at $j$
$z_{j} \in\{0,1\}$
Takes value 1 if facility $j$ is fortified
$x_{i j r} \in\{0,1\}$
Takes value 1 if retailer $i$ is assigned to facility $j$ at backup level $r$

There is a penalty cost $(\eta)$ per unit of lost demand. This cost is incurred either if it is too expensive to serve a customer from its nearest remaining operating facility, or if all primary and backup facilities are not available. Like the RFLP model presented in section (1.3.2), we handle this cost by including a dummy emergency facility $u$ in the model. As mentioned earlier, this emergency facility has no fixed cost and never fails, and the transportation cost from this facility to any customer is considered as $\eta$. 
Furthermore, like the joint location inventory model presented in section (1.3.1), weight factors $(\beta)$ and $(\theta)$ are added to the model for easiness of performing sensitivity analysis on the inventory and transportation costs, and for better observing the contribution of each of the costs in the model. The inventory control strategy in this work is based on the economic order quantity (EOQ) which is a popular approach for joint location inventory problems in the literature (e.g., Daskin et al. 2002; Shen et al. 2003). The expected total annual inventory (ordering + holding) cost for facility $j$ is as follows:

$$
\frac{\sum_{i} \sum_{r}\left(A_{j}+\beta g_{j}\right) \lambda_{i} x_{i j r}\left(1-q\left(1-z_{j}\right)\right) q^{r-1}}{Q_{j}}+\frac{h_{j} \theta Q_{j}}{2}
$$

The first term in the above equation is the expected annual ordering cost for facility $j$, since the expected annual demand for facility $j$ is $D_{j}=\sum_{i} \sum_{r} \lambda_{i} x_{i j r}\left(1-q\left(1-z_{j}\right)\right) q^{r-1}$. It is easy to observe that $\left(1-q\left(1-z_{j}\right)\right) q^{r-1}$ is the probability of serving retailer $i$ by facility $j$ at the assignment level $r$. The second term of equation (3.1) is the annual holding cost. For any solution, when the values of $x_{i j r}$ and $z_{j}$ are obtained, the optimal order quantity $\left(Q_{j}^{*}\right)$ at facility $j$ (if $y_{j}=1$ ) can be obtained using the EOQ model. By taking the derivative of equation (3.1) with respect to $Q_{j}$ and seting it to zero, the optimal order quantity for facility $j$ can be obtained as:

$$
Q_{j}^{*}=\sqrt{\frac{2\left(A_{j}+\beta g_{j}\right) \sum_{i} \sum_{r} \lambda_{i} x_{i j r}\left(1-q\left(1-z_{j}\right)\right) q^{r-1}}{h_{j} \theta}}
$$

Replacing the above optimal order quantity in the total annual inventory cost (equation 3.1), the optimal expected inventory cost for facility $j$ can be given as: 


$$
\operatorname{CINV}_{j}^{*}=\sqrt{2\left(A_{j}+\beta g_{j}\right) h_{j} \theta \sum_{i} \sum_{r} \lambda_{i} x_{i j r}\left(1-q\left(1-z_{j}\right)\right) q^{r-1}}
$$

The expected annual transportation cost for facility $j$ is also obtained as:

$$
\sum_{i} \sum_{r} \lambda_{i} \widehat{c_{i j}} x_{i j r}\left(1-q\left(1-z_{j}\right)\right) q^{r-1}
$$

Where $\widehat{c_{l j}}=\beta\left(c_{i j}+d_{j}\right)$.

The mathematical formulation is then:

P1:

$$
\begin{aligned}
& \text { Min } \quad \sum_{j} f_{j} y_{j}+\sum_{i}\left\{\sum_{r}\left[\sum_{j} \lambda_{i} \widehat{c_{i j}}\left(1-q\left(1-z_{j}\right)\right) q^{r-1} x_{i j r}+\lambda_{i} \eta q^{r-1} x_{i u r}\right]+\right. \\
& \left.\lambda_{i} \eta q^{R} x_{i u R+1}\right\}+\sum_{j} \sqrt{2\left(A_{j}+\beta g_{j}\right) h_{j} \theta \sum_{i} \sum_{r} \lambda_{i} x_{i j r}\left(1-q\left(1-z_{j}\right)\right) q^{r-1}}
\end{aligned}
$$

subject to:

$$
\begin{array}{ll}
\sum_{j} x_{i j r}+\sum_{s=1}^{r-1} \sum_{j} x_{i j s} z_{j}+\sum_{s=1}^{r} x_{i u s}=1 & ; \text { for } i=1, \ldots, I, r=1, \ldots, R \\
\sum_{r=1}^{R} \sum_{j} x_{i j r} z_{j}+\sum_{r=1}^{R+1} x_{i u r}=1 & ; \text { for } i=1, \ldots, I
\end{array}
$$




$$
\begin{array}{ll}
\sum_{r} x_{i j r} \leq y_{j} & ; \text { for } i=1, \ldots, I, j=1, \ldots, J \\
z_{j} \leq y_{j} & ; \text { for } j=1, \ldots, J \\
\sum_{j} S_{j} z_{j} \leq B & \\
x_{i j r}, y_{j}, z_{j} \in\{0,1\} & ; \text { for } i=1, \ldots, I, j=1, \ldots, J,
\end{array}
$$

In the above formulation, the objective function (equation 3.5) minimizes the total expected annual cost which includes the total cost of locating facilities (first term), transportation and lostsale (second term), and working inventory (third term) across all possible facility failure scenarios. The quantity $\left(1-q\left(1-z_{j}\right)\right) q^{r-1}$ in terms 2 and 3 of the objective function is the probability of assigning retailer $i$ to facility $j$ at back-up level $r$. To explain the quantiy $\lambda_{i} \eta q^{r-1} x_{\text {iur }}$ in term 2 of the objective function, it should be noted that to lose a customer at level $r, r-1$ facilities should be failed which may happen with the chance of $q^{r-1}$. However, this does not consider the case when all $\mathrm{R}$ levels of assignment for customer $i$ are to non-fortified facilities and all of them are nonoperational (which can happen with the chance of $q^{R}$ ). It is worth noting that this is different than other models in the literature such as the RFLP model proposed by Snyder and Daskin (2005) (section 1.3.2) and the study by Chen et al. (2011). The reason is that they did not account for fortification in their models, and thus, they could simply consider an expected lost-sale cost of $\sum_{i} \lambda_{i} \eta q^{R}$ for all of the customers. Since $\sum_{i} \lambda_{i} \eta q^{R}$ is a fixed cost, there is no need to consider that in the objective function. However, in the model used in this study, the $\operatorname{cost} \lambda_{i} \eta q^{R}$ should only be considered for customers that are not assigned to a fortified facility in any of the " $R$ " levels of the assignment. For this purpose, the term $\lambda_{i} \eta q^{R} x_{i u R+1}$ is included in the objective function and the set of constraints (3.7) is added. Constraints (3.6) guarantee that each retailer $i$ is assigned to a 
facility $j$ at each level $r$ provided that it has not been assigned either to a fortified facility or to the emergency facility $u$ in any of the previous $r-1$ levels. To explain constraints (3.7), let us assume that customer $i$ has not been assigned to a fortified facility (i.e. $\sum_{r=1}^{R} \sum_{j} x_{i j r} z_{j}=0$ ) and also this customer is never lost in any of the " $\mathrm{R}$ " levels of assignment that is $\sum_{j} x_{i j r}=1$ for $r=1, \ldots, R$ which enforces $\sum_{s=1}^{r} x_{i u s}=0$ for $r=1, \ldots, R$ by constraint (3.6). Since $\sum_{s=1}^{r} x_{i u s}=0$ for $r=$ $1, \ldots, R$ (i.e. $\sum_{r=1}^{R} x_{i u r}=0$ ), Constraint (3.7) enforces $x_{i u R+1}=1$, and as a result the quantity $\lambda_{i} \eta q^{R}$ for this customer (customer $i$ ) is considered in the objective function.

Constraint set (3.8) has two purposes. First, it ensures that a retailer is only assigned to an opened facility. Moreover, it assures that each retailer $i$ can be assigned to a facility $j$ at only one level of assignment. Constraints (3.9) guarantee that only opened facilities at each candidate location can be fortified. Constraints (3.10) enforce the total fortification budget, and Constraints (3.11) define the binary variables.

Problem (P1) is difficult to solve since there are two nonlinear terms in the objective function, term 2 and 3, and the quadratic nonlinear term $\left(x_{i j r} z_{j}\right)$ in constraint sets (3.6) and (3.7). Hence, it is desired for Problem (P1) to be reformulated and transformed to a more tractable problem. In the following chapter, this reformulation is first discussed, then two methodologies are presented for solving the problem. 


\section{Chapter 4 - Solution methodology}


This chapter provides two solution methodologies for the model described in chapter 3 . As mentioned in chapter 3, the model is hard to solve, and thus it needs to be transformed into a more tractable problem. The first section of this chapter describes how the proposed model is reformulated, and then two global search approaches are presented for solving the problem.

\subsection{Reformulation of the proposed model}

There are two nonlinear terms in the objective function of Problem (P1) in chapter 3, term 2 and 3. In addition, the quadratic nonlinear term $\left(x_{i j r} z_{j}\right)$ is presented in constraint sets (3.6) and (3.7). In the developed model, there are two sorts of nonlinearity functions: quadratic and square root. The latter includes the quadratic function as well, as it can be seen in the last term of the objective function. The term $x_{i j r}\left(1-q\left(1-z_{j}\right)\right)$ in the objective function can be simplified as:

$$
x_{i j r}\left(1-q\left(1-z_{j}\right)\right)=x_{i j r}(1-q)+q x_{i j r} z_{j}=(1-q) x_{i j r}+q x_{i j r} z_{j}
$$

Therefore, the first step is to substitute the quadratic term $\left(x_{i j r} z_{j}\right)$ with a new nonnegative variable $M_{i j r}$ employing a popular linearization method used in solving the quadratic assignment problem. To compensate for this substitution, the following three constraints are added to the model:

$$
\begin{array}{ll}
M_{i j r} \leq x_{i j r}, & ; \text { for } i=1, \ldots, I, j=1, \ldots, J, r=1, \ldots, R \\
M_{i j r} \leq z_{j}, & ; \text { for } i=1, \ldots, I, j=1, \ldots, J, r=1, \ldots, R \\
M_{i j r} \geq x_{i j r}+z_{j}-1, & ; \text { for } i=1, \ldots, I, j=1, \ldots, J, r=1, \ldots, R
\end{array}
$$




$$
M_{i j r} \geq 0 \quad ; \text { for } i=1, \ldots, I, j=1, \ldots, J, r=1, \ldots, R
$$

It should be noted that $M_{i j r}$ does not require an explicit integrality constraint as it is stated in (4.5), since equations (4.2-4.4) effectively constrain $M_{i j r}$ to be 1 when $x_{i j r}=z_{j}=1$, and 0 otherwise. Considering that $x_{i j r}$ is a binary variable and $M_{i j r}$ is inherently a binary variable (it can only take a value of 0 or 1 ), they are substituted by $x_{i j r}^{2}$ and $M_{i j r}^{2}$ under the square root term of the objective function. The reason for these substitutions will be discussed later in section (4.2).

Finally, by substituting the quantity $\sqrt{\sum_{i} \sum_{r} \lambda_{i} x_{i j r}\left(1-q\left(1-z_{j}\right)\right) q^{r-1}}$ in the last term in the objective function with a newly introduced positive variables $t_{j}$, problem (P1) can be reformulated as follows:

P2:

$\operatorname{Min} \sum_{j} f_{j} y_{j}+\sum_{i}\left\{\sum_{r}\left[\sum_{j \in J} \lambda_{i} \widehat{c_{i j}} q^{r-1}\left((1-q) x_{i j r}+q M_{i j r}\right)+\lambda_{i} \eta q^{r-1} x_{i u r}\right]+\right.$ $\left.\lambda_{i} \eta q^{R} x_{i u R+1}\right\}+\sum_{j} \sqrt{2\left(A_{j}+\beta g_{j}\right) h_{j} \theta} t_{j}$

subject to:

$$
\begin{aligned}
& \sqrt{\sum_{i} \sum_{r} \lambda_{i} q^{r-1}\left((1-q) x_{i j r}^{2}+q M_{i j r}^{2}\right)}-t_{j} \leq 0 \quad ; \text { for } j=1, \ldots, J \\
& \sum_{j} x_{i j r}+\sum_{r=0}^{r-1} \sum_{j} M_{i j r}+\sum_{s=1}^{r} x_{i u s}=1, \quad ; \text { for } i=1, \ldots, I, r=1, \ldots, R
\end{aligned}
$$




$$
\begin{array}{ll}
\sum_{r=1}^{R} \sum_{j} M_{i j r}+\sum_{r=1}^{R+1} x_{i u r}=1 & ; \text { for } i=1, \ldots, I \\
\sum_{r} x_{i j r} \leq y_{j}, & ; \text { for } i=1, \ldots, I, j=1, \ldots, J \\
z_{j} \leq y_{j} & ; \text { for } j=1, \ldots, J \\
\sum_{j} S_{j} z_{j} \leq B, & \\
M_{i j r} \leq x_{i j r}, & ; \text { for } i=1, \ldots, I, j=1, \ldots, J, r=1, \ldots, R \\
M_{i j r} \leq z_{j}, & ; \text { for } i=1, \ldots, I, j=1, \ldots, J, r=1, \ldots, R \\
M_{i j r} \geq x_{i j r}+z_{j}-1, & ; \text { for } i=1, \ldots, I, j=1, \ldots, J, r=1, \ldots, R \\
x_{i j r}, y_{j}, z_{j} \in\{0,1\} & ; \text { for } i=1, \ldots, I, j=1, \ldots, J, r=1, \ldots, R \\
M_{i j r} \geq 0, &
\end{array}
$$

It should be pointed out that constraint (4.7) in the above formulation is inherently an equality $(=)$ constraint. Since the coefficient for variable $\left(t_{j}\right)$ in the objective function is positive, and the objective function is a minimization, $\left(t_{j}\right)$ will be at its minimum in the optimal solution forcing the equality. Hence, an inequality $(\leq)$ constraint is used to set up a more efficient model. Additionally, constraint (4.7) is nonlinear and thus the reformulated model (P2) is a mixed integer nonlinear program (MINLP). The most basic form of an MINLP problem can be represented as follows: 
$\operatorname{Min} Z=f(x, y)$

subject to:

$$
\begin{aligned}
& g(x, y) \leq 0 \\
& x \in X, y \in Y
\end{aligned}
$$

Where $f(x, y)$ and $g(x, y)$ are both functions of $x$ and $y$ which are sets of discrete and continuous variables respectively. Methods that have addressed the solution of the MINLP problem include the branch and bound method (BB) (Dakin, 1965; Gupta and Ravindran, 1985; Nabar and Schrage, 1991; Leyffer, 2001), branch and cut (Stubbs and Mehrotra, 1999), Generalized Benders Decomposition (GBD) (Geoffrion, 1972), Outer-Approximation (OA) (Duran and Grossmann, 1986; Yuan et al., 1988; Leyffer, 1993; Fletcher and Leyffer, 1994), LP/NLP based branch and bound (Quesada and Grossmann, 1992), and Extended Cutting Plane Method (ECP) (Westerlund and Pettersson, 1995). Advances in today's computer technology have motivated many works to study the application of these algorithms in solving a variety of realworld problems. In this research, two solution methodologies are implemented to solve problem (P2). The first one is based on the Outer Approximation method, and the second is an algorithm that is mainly based on the LP/NLP based branch and bound method, which exploits more advanced features of the branch and bound framework. Similar ideas in the implementation of LP/NLP based branch and bound method were presented by Bonami et al. (2008), followed by Abhishek et al. (2010). The algorithm proposed in this research utilizes some of the features proposed by Abhishek et al. (2010) and customizes them to solve problem (P2). 


\subsection{Convexity proof for the nonlinear terms}

Before discussing the two proposed approaches, the following proposition provides a proof for convexity of nonlinear constraints (4.7). Since both approaches belong to the family of global search methods, the convexity assumption needs to be met in order to assure optimality.

Proposition: The function $g\left(x_{i j r}, M_{i j r}, t_{j}\right)=\sqrt{\sum_{i} \sum_{r} \lambda_{i} q^{r-1}\left((1-q) x_{i j r}^{2}+q M_{i j r}^{2}\right)}-t_{j}$, defined on the domain $[0,1] \times \mathbb{R}$, is convex.

Proof: As $t_{j}$ is linear, it is sufficient to prove that the square root term is convex. For this purpose, the Hessian matrix of the square root should be obtained. Let us label the square root term as follows:

$$
F=\sqrt{\sum_{r} \sum_{i} \lambda_{i} q^{r-1}\left((1-q) x_{i j r}^{2}+q M_{i j r}^{2}\right)}
$$

The Hessian matrix can then be obtained as:

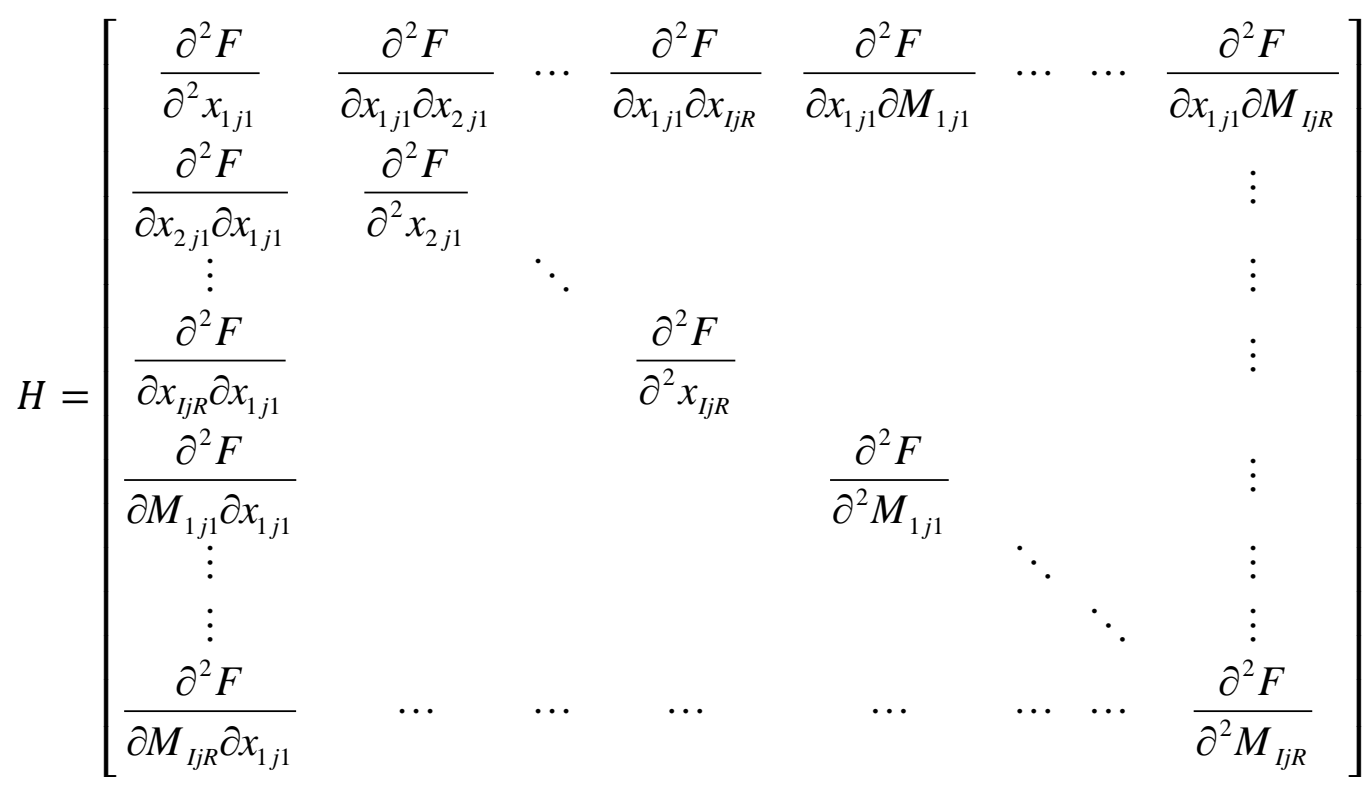


To prove the convexity of $F$, it is sufficient to show that the Hessian matrix is positive semi-definite. There are different ways to prove that a matrix is positive semi-definite. The approach used here is to show that the Hessian matrix is a diagonally dominant matrix. A matrix is diagonally dominant if the absolute value of each of its diagonal elements is bigger than or equal to the summation of the absolute values of all the elements in the same row (column). Based on Gershgorin circle theorem, a symmetric diagonally dominant real matrix with nonnegative diagonal entries is positive semi-definite. For this purpose, the first diagonal element $\left(\frac{\partial^{2} F}{\partial^{2} x_{1 j 1}}\right)$ is selected to investigate whether it is bigger than or equal to the summation of the first row elements. To make it easier to follow, let us extend the summations inside $F$ as follows:

$$
\begin{aligned}
& F=\sqrt{\sum_{i} \sum_{r} \lambda_{i} q^{r-1}\left((1-q) x_{i j r}^{2}+q M_{i j r}^{2}\right)} \\
& =\sqrt{\lambda_{1}\left((1-q) x_{1 j 1}^{2}+q M_{1 j 1}^{2}\right)+\lambda_{2}\left((1-q) x_{2 j 1}^{2}+q M_{2 j 1}^{2}\right)+\cdots+\lambda_{I} q^{R-1}\left((1-q) x_{I j R}^{2}+q M_{I j R}^{2}\right)}
\end{aligned}
$$

The first and the second derivatives of $\mathrm{F}$ with respect to $x_{1 j 1}$ can be obtained as follows:

$$
\begin{aligned}
\frac{\partial F}{\partial x_{1 j 1}}= & \frac{1}{2} \times \frac{2 \lambda_{1}(1-q) x_{1 j 1}}{F}=\frac{\lambda_{1}(1-q) x_{1 j 1}}{F} ; \\
\frac{\partial^{2} F}{\partial^{2} x_{1 j 1}} & =\frac{\lambda_{1}(1-q) F-\frac{\lambda_{1}^{2}(1-q)^{2} x_{1 j 1}{ }^{2}}{F}}{F^{2}}=\frac{\frac{\lambda_{1}(1-q) F^{2}-\lambda_{1}^{2}(1-q)^{2} x_{1 j 1}{ }^{2}}{F}}{F^{2}} \\
& =\frac{\lambda_{1}(1-q)\left[F^{2}-\left(\lambda_{1}(1-q)\right) x_{1 j 1}^{2}\right]}{F^{3}} ;
\end{aligned}
$$


Replacing $F^{2}$ with $\sum_{i} \sum_{r} \lambda_{i} q^{r-1}\left((1-q) x_{i j r}^{2}+q M_{i j r}^{2}\right)$ and extending the summations, the second derivative can be obtained as follows:

$$
\begin{aligned}
& \frac{\partial^{2} F}{\partial^{2} x_{1 j 1}}= \\
& \frac{\lambda_{1}(1-q)\left[\left(\lambda_{2}(1-q)\right) x_{2 j 1}^{2}+\left(\lambda_{3}(1-q)\right) x_{3 j 1}^{2}+\cdots+\left(\lambda_{I}(1-q)\right) x_{I j R}^{2}+\left(\lambda_{1}(q)\right) M_{1 j 1}^{2}+\cdots+\left(\lambda_{i}(q)\right) M_{I j R}^{2}\right]}{F^{3}}
\end{aligned}
$$

Considering that $\frac{\partial F}{\partial x_{1 j 1}}=\frac{\lambda_{1}(1-q) x_{1 j 1}}{F}$, other elements in the first row such as $\frac{\partial^{2} F}{\partial x_{1 j 1} \partial x_{2 j 1}}$ can be obtained as:

$\frac{\partial^{2} F}{\partial x_{1 j 1} \partial x_{2 j 1}}=\frac{0-\lambda_{1}(1-q) x_{1 j 1} \times \frac{\lambda_{2}(1-q) x_{2 j 1}}{F}}{F^{2}}=-\frac{\lambda_{1}(1-q) x_{1 j 1}\left(\lambda_{2}(1-q) x_{2 j 1}\right)}{F^{3}}$

The above equation is the same for all other partial derivatives in the first row, except for the term $\lambda_{2}(1-q) x_{2 j 1}$ which is substituted by the corresponding term using the second variable in the partial derivative. In general the coefficient for $x_{i j r}(i, r \neq 1)$ is $\left(\lambda_{i}(1-q)\right)$, and the coefficient for $M_{i j r}$ is $\left(\lambda_{i}(q)\right)$. In other words, the non-diagonal elements in the first row can be obtained as follows:

$$
\left\{\begin{array}{c}
\frac{\partial^{2} F}{\partial x_{1 j 1} \partial x_{i j r}}=-\frac{\lambda_{1}(1-q) x_{1 j 1}\left[\left(\lambda_{i}(1-q)\right) x_{i j r}\right]}{F^{3}} \quad i, r \neq 1 \\
\frac{\partial^{2} F}{\partial x_{1 j 1} \partial M_{i j r}}=-\frac{\lambda_{1}(1-q) x_{1 j 1}\left[\left(\lambda_{i}(q)\right) M_{i j r}\right]}{F^{3}}
\end{array}\right.
$$


The sum of the absolute values of these non-diagonal elements in row 1 yields the following quantity:

$\frac{\lambda_{1}(1-q) x_{1 j 1}\left[\left(\lambda_{2}(1-q)\right) x_{2 j 1}+\left(\lambda_{3}(1-q)\right) x_{3 j 1}+\cdots+\left(\lambda_{I}(1-q)\right) x_{I j R}+\cdots+\left(\lambda_{i}(q)\right) M_{I j R}\right]}{F^{3}}$

Since $x_{i j r}^{2}=x_{i j r}$, and $M_{i j r}^{2}=M_{i j r}$, the only difference between the above equation and the diagonal value $\left(\frac{\partial^{2} F}{\partial^{2} x_{1 j 1}}\right)$ is the element $x_{1 j 1}$. Considering that $x_{1 j 1}$ is a binary variable, the diagonal element is greater than the sum of all other elements in the first row when $x_{1 j 1}=0$, and the two values are equal when $x_{1 j 1}=1$. The same logic can be used to prove that all the diagonal elements of the Hessian matrix are greater than or equal to the sum of the absolute values of the other elements in the corresponding rows. Hence, it can be concluded that the Hessian matrix is a symmetric diagonally dominant matrix, and it is easy to observe that the diagonal entries are nonnegative, and thus the matrix is a positive semi-definite. QED

\subsection{Outer Approximation}

Outer Approximation (OA), originally proposed by Duran and Grossman (1986), is recognized in the literature as one of the strongest methods for solving MINLPs. OA works by decomposing the MINLP problem into a nonlinear program (NLP) which is referred to as a subproblem (SP) and a mixed integer linear program (MILP) which is considered as a master problem. The NLP sub problem is obtained by fixing the integer variables in the original MINLP model, and when the solution of the NLP sub problem is feasible, it provides an upper bound for the MINLP problem. The OA master problem substitutes the nonlinear functions in the MINLP problem with supporting hyperplanes (the linearization of the nonlinear terms); and thus, the master problem is a relaxed linear representation of the MINLP, and it gives a lower bound for the 
MINLP. Hence, the method operates by iteratively solving a series of NLP sub problems, and MILP master problems until the difference between the lower and upper bounds provided by the master and the sub problem is sufficiently small. This representation of the OA algorithm was presented by Duran and Grossman (1986). Later, Leyffer (1993), followed by Fletcher and Leyffer (1994), proposed a different version of OA where the MILP master problems are not needed to be solved to optimality. This is specifically useful since, as it will be explained, the size of the master problem keeps on increasing with the accumulation of the linearization cuts at each iteration of the algorithm; and it is not practical to solve the master problem to optimality for large instance problems. The OA method works best, and provides global optimal solutions, when the nonlinear terms are convex. However, there are some approaches in the literature that handle the nonconvexities (e.g. Viswanathan and Grossmann, 1990, and Grossmann and Kravanja, 1997).

A comprehensive literature on the OA method may be found in Duran and Grossman (1986), Leyffer (1993), Grossmann (2002), and Belotti et al. (2012). The following sub-sections describe how the OA method is used for the problem on-hand (P2).

\subsubsection{Sub problem}

Given fixed values for the integer variables at each iteration of OA algorithm, the OA sub problem finds the optimal value for the continuous variables, providing a feasible point to the main MINLP problem. Later in the master problem, the linearization about this feasible point is used to approximate the nonlinear constraints set (4.7) in problem (P2). In the case of model (P2), there is only one set of continuous variables $\left(t_{j}\right)$ in the constraint set (4.7). Noting that the objective function is a minimization and the coefficient of the variable $\left(t_{j}\right)$ in the objective function is 
positive, the optimal values for the continuous variables $\left(t_{j}\right)$, given fixed values for integer variables: $\hat{x}_{i j r}^{k}, \hat{y}_{j}^{k}, \hat{z}_{j}^{k}$ at each iteration $k$, can be obtained in a closed form as follows:

$$
\hat{t}_{j}^{k}=\sqrt{\sum_{i} \sum_{r} \lambda_{i} q^{r-1}\left((1-q) \hat{x}_{i j r}^{k}+q \widehat{M}_{i j r}^{k}\right)} \quad ; \text { for } j=1, \ldots, J
$$

It should be noted that, as mentioned earlier, the constraint sets (4.2-4.4) force $\widehat{M}_{i j r}^{k}$ to equal $\hat{x}_{i j r}^{k} \hat{Z}_{j}^{k}$

Thus, the NLP sub problem can be trivially solved, yielding the OA upper bound as follows:

$$
\begin{aligned}
& V^{k}=\sum_{j} f_{j} \hat{y}_{j}^{k}+\sum_{i}\left\{\sum_{r}\left[\sum_{j \in J} \lambda_{i} \widehat{c_{i j}} q^{r-1}\left((1-q) \hat{x}_{i j r}^{k}+q \widehat{M}_{i j r}^{k}\right)+\lambda_{i} \eta q^{r-1} \hat{x}_{i u r}^{k}\right]+\right. \\
& \left.\lambda_{i} \eta q^{R} \hat{x}_{i u R+1}^{k}\right\}+\sum_{j} \sqrt{2\left(A_{j}+\beta g_{j}\right) h_{j} \theta} \hat{t}_{j}^{k}
\end{aligned}
$$

A similar situation was found in the literature in a paper by Shahabi et. al. (2014). It should also be noted that, the OA requires an initial integer feasible solution to be used at the beginning of the algorithm. An initial solution can be obtained by eliminating the nonlinear inventory term $\sum_{j} \sqrt{2\left(A_{j}+\beta g_{j}\right) h_{j} \theta} t_{j}$ from the objective function, along with the nonlinear constraint (4.7) associated with it, and solving the resultant model.

\subsubsection{Master problem}

The master problem derives the linear approximation of the nonlinear constraint (4.7) about the feasible point obtained by the NLP sub problem at each iteration of the OA algorithm. More 
specifically, considering $g\left(x_{i j r}, M_{i j r}, t_{j}\right)=\sqrt{\sum_{i} \sum_{r} \lambda_{i} q^{r-1}\left((1-q) x_{i j r}^{2}+q M_{i j r}^{2}\right)}-t_{j}$, the linear approximation of constraints set (4.7) about the point $\hat{x}_{i j r}^{k}, \hat{y}_{j}^{k}, \hat{z}_{j}^{k}, \hat{t}_{j}^{k}$, and $\widehat{M}_{i j r}^{k}$ at each iteration $k$ can be obtained as follows:

$$
g\left(\hat{x}_{i j r}^{k}, \widehat{M}_{i j r}^{k}, \hat{t}_{j}^{k}\right)+\left[\nabla g\left(\hat{x}_{i j r}^{k}, \widehat{M}_{i j r}^{k}, \hat{t}_{j}^{k}\right)\right]^{T}\left[\begin{array}{c}
x_{i j r}-\hat{x}_{i j r}^{k} \\
M_{i j r}-\widehat{M}_{i j r}^{k} \\
t_{j}-\hat{t}_{j}^{k}
\end{array}\right] \leq 0, \quad ; \text { for } j=1, \ldots, J
$$

which can be written as:

$$
\begin{aligned}
& \sqrt{\sum_{i} \sum_{r} \lambda_{i} q^{r-1}\left((1-q) \hat{x}_{i j r}^{k}{ }^{2}+q \widehat{M}_{i j r}^{k}{ }^{2}\right)}-\hat{t}_{j}^{k}+ \\
& {\left[\frac{\sum_{i} \sum_{r} \lambda_{i} q^{r-1}(1-q) \hat{x}_{i j r}^{k}}{\sqrt{\sum_{i} \sum_{r} \lambda_{i} q^{r-1}\left((1-q) \hat{x}_{i j r}^{k}{ }^{2}+q \widehat{M}_{i j r}^{k}{ }^{2}\right)}} \frac{\sum_{i} \sum_{r} \lambda_{i} q^{r} \widehat{M}_{i j r}^{k}}{\sqrt{\sum_{i} \sum_{r} \lambda_{i} q^{r-1}\left((1-q) \hat{x}_{i j r}^{k}+q \widehat{M}_{i j r}^{k}{ }^{2}\right)}}-1\right]} \\
& {\left[\begin{array}{c}
x_{i j r}-\hat{x}_{i j r}^{k} \\
M_{i j r}-\widehat{M}_{i j r}^{k} \\
t_{j}-\hat{t}_{j}^{k}
\end{array}\right] \leq 0 \quad ; \text { for } j=1, \ldots, J}
\end{aligned}
$$

The first term in the above equation will be cancelled out since $\hat{t}_{j}^{k}=\sqrt{\sum_{i} \sum_{r} \lambda_{i} q^{r-1}\left((1-q) \hat{x}_{i j r}^{k}+q \widehat{M}_{i j r}^{k}\right)}$ from equation (4.21), and by performing the matrix multiplication in the second term, the equation can be written as follows:

$$
\frac{\sum_{i} \sum_{r} \lambda_{i} q^{r-1}(1-q) \hat{x}_{i j r}^{k}\left(x_{i j r}-\hat{x}_{i j r}^{k}\right)}{\sqrt{\sum_{i} \sum_{r} \lambda_{i} q^{r-1}\left((1-q) \hat{x}_{i j r}^{k}{ }^{2}+q \widehat{M}_{i j r}^{k}{ }^{2}\right)}}+
$$




$$
\frac{\sum_{i} \sum_{r} \lambda_{i} q^{r} \widehat{M}_{i j r}^{k}\left(M_{i j r}-\widehat{M}_{i j r}^{k}\right)}{\sqrt{\sum_{i} \sum_{r} \lambda_{i} q^{r-1}\left((1-q) \hat{x}_{i j r}^{k}+q \widehat{M}_{i j r}^{k}\right)}}-t_{j}+\hat{t}_{j}^{k} \leq 0 \quad ; \text { for } j=1, \ldots, J
$$

It should be noted that all the variables inside the square roots of constraint (4.25) are fixed to the values obtained in iteration $k$, and thus the constraint is linear. It should also be noted that this linearization is performed at each iteration $k$, using the point obtained from the solution of the NLP sub problem. Therefore, the size of the master problem is constantly increased since the linearizations are accumulated as the iterations $(k=1, \ldots, K)$ continue.

Finally, the OA master problem can be given as follows:

MP:

$$
\begin{aligned}
& \operatorname{Min} \operatorname{MOF}^{k}=\sum_{j} f_{j} y_{j}+\sum_{i}\left\{\sum _ { r } \left[\sum_{j \in J} \lambda_{i} \widehat{c_{i j}} q^{r-1}\left((1-q) x_{i j r}+q M_{i j r}\right)+\right.\right. \\
& \left.\left.\lambda_{i} \eta q^{r-1} x_{i u r}\right]+\lambda_{i} \eta q^{R} x_{i u R+1}\right\}+\sum_{j} \sqrt{2\left(A_{j}+\beta g_{j}\right) h_{j} \theta} t_{j}
\end{aligned}
$$

subject to:

$$
\begin{array}{lc}
\frac{\sum_{i} \sum_{r} \lambda_{i} q^{r-1}(1-q) \hat{x}_{i j r}^{l}\left(x_{i j r}-\hat{x}_{i j r}^{l}\right)}{\sqrt{\sum_{i} \sum_{r} \lambda_{i} q^{r-1}\left((1-q) \hat{x}_{i j r}^{l}{ }^{2}+q \widehat{M}_{i j r}^{l}{ }^{2}\right)}}+\frac{\sum_{i} \sum_{r} \lambda_{i} q^{r} \widehat{M}_{i j r}^{l}\left(M_{i j r}-\widehat{M}_{i j r}^{l}\right)}{\sqrt{\sum_{i} \sum_{r} \lambda_{i} q^{r-1}\left((1-q) \hat{x}_{i j r}^{l}{ }^{2}+q \widehat{M}_{i j r}^{l}{ }^{2}\right)}} & ; \text { for } j=1, \ldots, J, l=1, \ldots, k \\
-t_{j}+\hat{t}_{j}^{k} \leq 0 & \quad ; \text { for } l=1, \ldots, k \\
\sum_{i, j, r \in S^{l}} x_{i j r}-\sum_{i, j, r \in S^{\prime l}} x_{i j r} \leq\left|S^{l}\right|-1 & \\
M O F^{k} \leq U B^{k}-\varepsilon &
\end{array}
$$

And constraint sets (4.8) - (4.17) 
In the above formulation, constraint sets (4.27) are the linearization cuts discussed earlier, and equation (4.28) is an integer cut, which goal is to make it infeasible for the integer variables $x_{i j r}$ to take the same values generated at any of the previous $k-1$ iterations. In other words, the integer cut ensures that new integer values are generated at each iteration $k$. In this equation, $S^{l}=$ $\left\{(i, j, r) \mid \hat{x}_{i j r}^{l}=1\right\}$ and $S^{\prime l}=\left\{(i, j, r) \mid \hat{x}_{i j r}^{l}=0\right\}$ at each iteration $l$. In addition, $\left|S^{l}\right|$ represents the cardinality of $S^{l}$ at iteration $l$. It is worth noting that the integer variables $y_{j}$ and $z_{j}$ are not included in this integer cut since their values change only if there is a change in the values of $x_{i j r}$. Constraint (4.29) is considered to use the OA version proposed by Leyffer (1993) and Fletcher and Leyffer (1994). In this case, the master problem does not need to be solved to optimality, and the algorithm stops when it cannot generate a feasible solution with an objective function value (MOF) less than the current upper bound $\left(U B^{k}=\min _{k}\left\{V^{k}\right\}\right)$ minus the tolerance $\varepsilon$. Needless to say, the algorithm is terminated only when the master problem becomes infeasible. The infeasibility of the master problem, in this case, means that the integer space cannot be searched further, and the solution at the current upper bound is optimal or within a distance of $\varepsilon$ from the optimal, which is called an $\varepsilon$-optimal solution to the primary problem.

Based on the above explanations, the OA algorithm used to solve problem (P2) in this research involves successively solving the NLP sub problems and the MILP master problems. At each iteration $k$, the NLP problem provides a feasible point to the primary MINLP problem (P2). Then the nonlinear constraint (4.7) in problem (P2) is linearized about the feasible point generated by the NLP problem, and a new set of linearization cuts (constraint (4.27)) is added to the master problem (MP). The solution of the MILP master problem provides new integer values which are used to solve the NLP sub problem in the following iteration. This procedure is repeated until the 
master problem becomes infeasible. A description of the algorithm steps are illustrated in table (4.1).

Table 4.1. Outer Approximation (OA) algorithm steps

Choose a maximum number of iterations $K$, and $\varepsilon>0$

Step 0: Set UB $=+\infty, k=1$, and Solve model (P2) without its nonlinear inventory counterparts to obtain initial values of $\widehat{\boldsymbol{y}}_{\boldsymbol{j}}^{\mathbf{1}}, \widehat{\mathbf{z}}_{\boldsymbol{j}}^{\mathbf{1}}, \hat{x}_{i j r}^{1}$ and $\widehat{\boldsymbol{M}}_{\boldsymbol{i j r}}^{\mathbf{1}}$.

Step 1: Solve the NLP sub-problem by evaluating the continuous variable $\hat{\boldsymbol{t}}_{\boldsymbol{j}}^{\boldsymbol{k}}$ according to equation (4.21), followed by obtaining the sub problem objective function $\boldsymbol{V}^{\boldsymbol{k}}$ according to equation (4.22).

Step 2: If $\left(\boldsymbol{V}^{\boldsymbol{k}}<\mathbf{U B}\right)$, update the upper bound and update the current best points as $\boldsymbol{y}_{\boldsymbol{j}}^{*}=\widehat{\boldsymbol{y}}_{\boldsymbol{j}}^{\boldsymbol{k}}$, $x_{i j r}^{*}=\widehat{x}_{i j r}^{k}, z_{j}^{*}=\widehat{z}_{j}^{k}, M_{i j r}^{*}=\widehat{M}_{i j r}^{k}, t_{j}^{*}=\hat{t}_{j}^{k}$

Step 3: Add equation (4.27-4.29) for iteration $k$ and solve the master problem (MP). If the master problem is feasible and $\boldsymbol{k} \leq \boldsymbol{K}$, go to step 4. Otherwise, go to step 5 .

Step 4: let the solution of the master problem be $\widehat{\boldsymbol{x}}_{\boldsymbol{i} \boldsymbol{j} \boldsymbol{r}}^{\boldsymbol{k}+\mathbf{1}}, \widehat{\boldsymbol{y}}_{\boldsymbol{j}}^{\boldsymbol{k}+\mathbf{1}}, \hat{\boldsymbol{z}}_{\boldsymbol{j}}^{\boldsymbol{k}+\mathbf{1}}$, set $\boldsymbol{k}=\boldsymbol{k}+\mathbf{1}$ and go back to step 1 .

Step 5: Terminate the algorithm, and report the UB, and $\boldsymbol{y}_{\boldsymbol{j}}^{*}, \boldsymbol{x}_{\boldsymbol{i j} \boldsymbol{r}}^{*}, \boldsymbol{z}_{\boldsymbol{j}}^{*}, \boldsymbol{M}_{\boldsymbol{i j r}}^{*}, \boldsymbol{t}_{\boldsymbol{j}}^{*}$ as the optimal solution. 


\subsection{LP/NLP based branch and bound framework}

LP/NLP-based branch-and-bound (LP/NLP-BB) method, introduced by Quesada and Grossmann (1992), has emerged as a powerful class of algorithms for solving convex MINLP problems. The methodology has been viewed as an improvement to the Outer Approximation approach by avoiding the solution of multiple MILP master problems, which take an increasing amount of computation time (Belotti et al. (2012). As discussed in section (4.3), new linearization cuts are generated for the MILP master problem (MP) at each iteration of the Outer Approximation algorithm, and therefore the size of the MILP master problem increases with the number of iterations. In fact, the LP/NLP-BB algorithm has been motivated by the fact that an increasing amount of computation time is spent by the Outer Approximation in solving successive MILP master problems. Furthermore, these MILP master problems are usually related to one another; and thus, considerable amount of information is regenerated each time a MILP master problem is solved.

The LP/NLP-BB algorithm intelligently avoids repetitions in the solution of the MILP master problem, and instead solves only one MILP problem which is updated as new integer solutions are found during the tree search of the branch and bound algorithm used to solve the MILP master problem.

For more clarifications, one should note that each MILP master problem is solved by using a branch and bound algorithm. The branch and bound method, in brief, starts by solving a continuous Linear Programming (LP) relaxation of the MILP. If all discrete variables take integer values the search is stopped. Otherwise, a tree search is performed in the space of the integer variables, during which, the integer variables are successively fixed at the corresponding nodes of the tree resulting in the generation of new relaxed LP problems in the offspring nodes. The solution 
of these LP relaxations yield lower bounds for the primary MILP problem. When all integer variables take discrete values at the solution of a LP problem, it yields an upper bound to the primary MILP problem. In addition, the corresponding node is fathomed. Fathoming of nodes also occurs when the lower bound of a node exceeds the current upper bound, or when the corresponding LP relaxation is infeasible. The branch and bound algorithm terminates whenever the stack of the LP relaxation problems is emptied (all the nodes are fathomed) or a predetermined gap between the lower and upper bound is reached. More information on branch and bound algorithm can be found in the literature (e.g. Beale 1985, or Johnson et al. 2000).

Since OA solves a MILP master problem at each iteration of the algorithm, and consequently multiple MILPs are solved using the branch and bound method, the algorithm has been considered as a multi-tree approach (Belotti et al. 2012). LP/NLP-BB, on the other hand, solves only one MILP for the entire algorithm where the NLP sub problem is solved whenever a new integer solution is found during the branch and bound tree search, and all the LP relaxation problems at open nodes are updated with new linearization cuts obtained from the solution of the NLP sub problem. Hence, LP/NLP-BB algorithm integrates the NLP sub problem into the (only one) branch and bound tree search used for solving the MILP problem, and thus; it is viewed as a single-tree approach.

\subsubsection{Implementation of LP/NLP-BB for problem (P2)}

To apply LP/NLP-BB method for problem (P2), first, like the OA algorithm, model (P2) is solved without its nonlinear inventory counterparts in order to obtain initial values for the integer values, and consequently the initial linearization cuts are generated at the solution of the NLP sub problem. Then, the MILP master problem is solved by a branch and bound framework where each 
time an integer solution is obtained at the continuous LP relaxation of the MILP master problem (MP), the branch and bound algorithm is interrupted to solve the NLP sub problem, which is then followed by the generation of new linearization cuts at the solution of the NLP sub problem. These new linearization cuts are then added to all the remaining LP relaxation problems of MP to update the branch and bound tree at each open node. The LP relaxation of the MILP master problem is obtained by replacing constraint (4.16) in the MP problem by its continuous relaxation for the binary variables. So, $x_{i j r}, y_{j}, z_{j} \in\{0,1\}$ are replaced with $0 \leq x_{i j r} \leq 1,0 \leq y_{j} \leq 1,0 \leq z_{j} \leq 1$. The LP relaxation problem is termed LPR-MP in this research.

The algorithm terminates similar to the regular branch and bound algorithm; either when all nodes are fathomed, or when a predetermined termination criterion, such as a tolerance gap between lower and upper bounds, or the maximum number of nodes, is reached.

It should be noted that when the LPR-MP problem in a node yields an integer solution, unlike the ordinary branch and bound method, the node should not be fathomed. The reason is that when the new linearization cuts, generated at the solution of the NLP sub problem, are added to the LPR-MP problem, the integer solution may no longer be feasible for the updated LPR-MP problem at the node, as it has been cut out by the new linearization cuts. Hence, fathoming of nodes in the LP/NLP-BB method occurs either when the LPR-MP problem is infeasible, or when the lower bound of the nodes exceeds the current upper bound. It should be noted that the solution of the NLP sub problem yields an upper bound for the primary problem (P2). Each time the NLP sub problem is solved, the upper bound is updated, and consequently any node on the stack, having a lower bound greater than or equal to the updated upper bound, is fathomed.

It is worth noting that, as mentioned by Grossmann (2002), the advantage of using LP/NLP-BB algorithm over the OA approach comes with the cost that the number of NLP sub 
problems to be solved during the algorithm may increase. Thus, the LP/NLP-BB algorithm is more efficient for problems where the MILP master problem constitutes the bottleneck of the algorithm. This is obviously the case for problem (P2) since, as mentioned in section (4.2.1), the NLP sub problem can be trivially solved using the special structure of the problem. Therefore, the MILP master problem is considered as the bottleneck of the algorithm.

The LP/NLP-BB algorithm used in this research is presented in table (4.2). It should be noted that, unlike the OA method described in section (4.3), index $k$ does not refer to the number of iterations, and it is used to track the number of times the LPR-MP problem is solved. The primal heuristic and the linearization generation used in the algorithm will be discussed in section (4.4.2). As mentioned earlier, the LP/NLP-BB algorithm used to solve problem (P2), exploits some advanced features of the branch and bound framework. These features are described in the following sub-section.

\subsubsection{Branch and Bound features}

Abhishek et al. (2010) have shown that the LP/NLP-BB algorithm proposed by Quesada and Grossmann (1992) can be significantly improved by implementing it within a modern MILP solver which uses advanced features of a branch and bound framework such as preprocessing, primal heuristics, branching and node selection rules. They also showed that it is advantageous to generate linearization cuts about fractional solutions obtained at the LP relaxation problem. This was a breakthrough in the literature of using the LP/NLP-BB algorithm proposed by Quesada and Grossmann (1992). All work in the literature prior to Abhishek et al. (2010) (e.g. Bonami et al., 2008) considered just the original way of linearization generation in the algorithm which occurs only once an integer solution is found at any node of the branch and bound, and no work noticed 
Table 4.2. LP/NLP based Branch and Bound (LP/NLP-BB) algorithm

Determine termination criteria: an optimality gap (gap between Lower bound and Upper bound) and a maximum number of nodes.

\section{Initialization:}

Step 1: Set UB $=+\infty, k=0$ and Solve model (P2) without its nonlinear inventory counterparts to obtain initial values of $\hat{y}_{j}^{0}, \hat{z}_{j}^{0}, \hat{x}_{i j r}^{0}$ and $\widehat{M}_{i j r}^{0}$.

Step 2: Solve the NLP sub problem by evaluating the continuous variable $\hat{t}_{j}^{k}$ according to equation (4.21), followed by obtaining the sub problem objective function $V^{k}$ according to equation (4.22).

Step 3: If $\left(V^{k}<U B\right)$, update the upper bound $\left(U B=V^{k}\right)$ and update the current best points as $y_{j}^{*}=\hat{y}_{j}^{k}, x_{i j r}^{*}=\hat{x}_{i j r}^{k}, z_{j}^{*}=\hat{z}_{j}^{k}, M_{i j r}^{*}=\widehat{M}_{i j r}^{k}, t_{j}^{*}=\hat{t}_{j}^{k}$.

Step 4: Add linearization cuts according to equations (4.27-4.29).

while (the stack of remaining open nodes is not empty) And (None of the termination criteria has been met) do

\section{Node selection:}

Select (as detailed in section 4.4.2.3) and remove a node from the stack of remaining open nodes, and set $k=k+1$.

\section{Evaluation:}

Solve the LPR-MP problem for the selected node. (Continued in the next page...) 


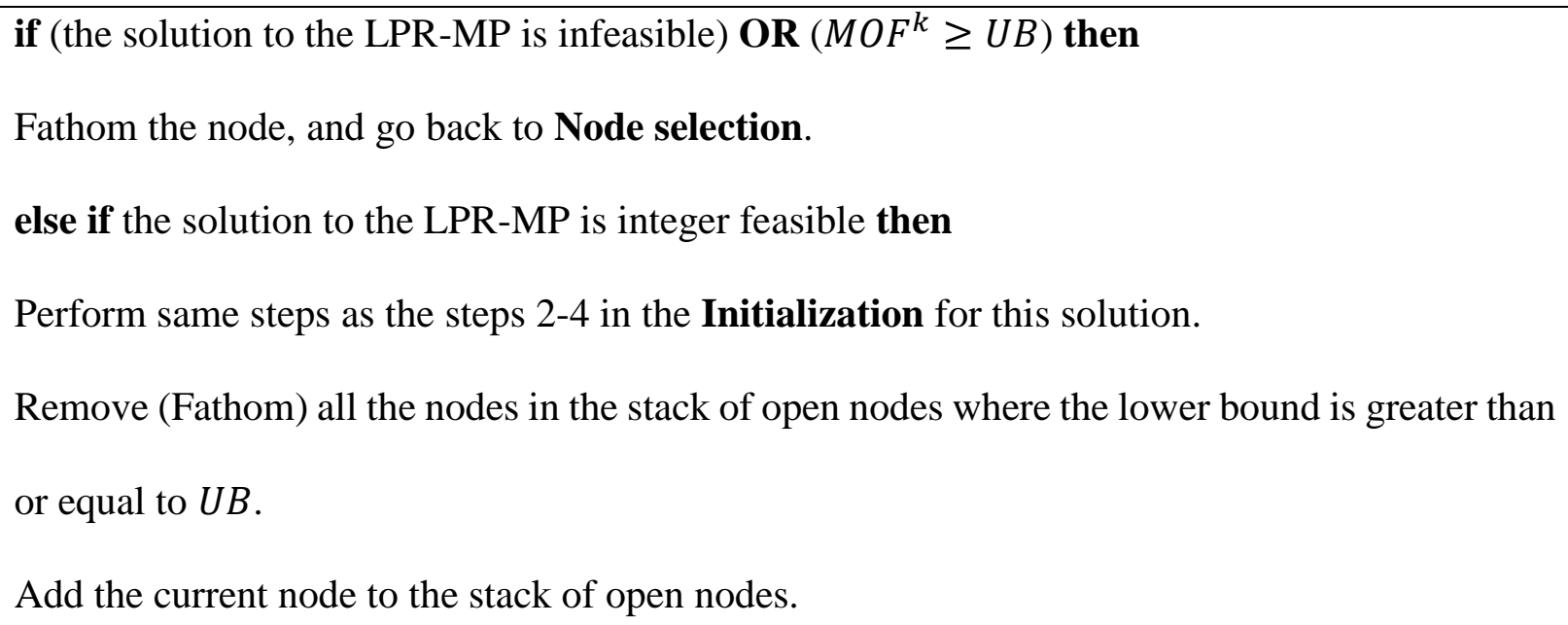

\section{Linearization cuts at fractional solution:}

else if Perform linearization generation at fractional solution (See table 4.4)

Go to Evaluation.

else

\section{Primal Heuristic:}

Perform Primal Heuristic (RINS) (See table 4.3).

Perform same steps as the steps 2-4 in the Initialization for the solution provided by RINS.

Remove (Fathom) all the nodes in the stack of open nodes, the lower bound of which are greater than or equal to $U B$.

\section{Branching:}

Select a variable for branching.

end if

end while 
this flexibility in generating linearization cuts within the LP/NLP-BB framework. The implementation of LP/NLP-BB algorithm in this research follows Abhishek et al. (2010) work and generates linearization cuts at fractional solutions obtained at LPR-MP problem. In the following sub-sections, the branch and bound features used within the LP/NLP-BB framework are outlined.

\subsubsection{Preprocessing}

One of the effective techniques used in modern MILP solvers is preprocessing. According to Savelsbergh (1994), the goal of preprocessing is to investigate each one of the constraints within the system of inequalities of the problem to check the following: whether the inequality is redundant, whether the bounds on the variables can be improved by using the inequality, whether the inequality can be strengthened by modifying its matrix coefficients, and whether the inequality forces some of the binary variables to either zero or one. All of these checks help to increase the bound obtainable from the solution of the LP relaxation of the MILP at each node of the branch and bound.

A variety of preprocessing techniques are described in detail in the work by Savelsbergh (1994). As CPLEX solver is used to solve the LP relaxations (LPR-MP) problem in this research, the preprocessing techniques within CPLEX are exploited for the LP/NLP-BB algorithm used to solve problem (P2).

\subsubsection{Primal heuristic}

Primal heuristic is one of the most useful features of a branch and bound framework that is used to quickly find good feasible (integer) solutions. Obtaining a high quality integer solution at the beginning of the branch and bound tree search, results in a strong upper bound on the optimal 
solution value, which can significantly reduce the number of nodes that must be enumerated in a branch and bound algorithm. As Abhishek et al. (2010) mentioned, this is of special importance for the case of LP/NLP-BB algorithm since only at the integer feasible solutions, the NLP sub problem can be solved, and consequently the upper bound is updated and new linearization cuts are generated. Abhishek et al. (2010) conducted an experiment in an effort to investigate the effectiveness of the primal heuristic, and other features such as preprocessing, in the context of LP/NLP-BB algorithm in solving a set of MINLP problems. They concluded that the primal heuristic has a significant impact in solving the MINLP instances compared to other features. While Abhishek et al. (2010) used an iterative diving-based primal heuristic within MINTO (the MILP solver used in their work), the primal heuristic used in this research is based on the Relaxation Induced Neighborhood Search (RINS) method introduced by Danna et al. (2005).

RINS is an improvement heuristic which is based on combining two types of solution faced during the branch and bound tree search: The integer solution which is feasible but often far from the optimal solution, and the solution of the continuous LP relaxation (fractional solution) at the current node which has a better objective value than that of the integer solution but is not feasible with respect to the integrality constraints. RINS intuitively combines the neighborhoods of both solutions hoping that it can achieve a feasible integer solution that has a better objective value. According to the method, the variables that differ in the two solutions are the ones that deserve more attention, and thus the variables that take the same values in the two solutions are fixed and a sub-MILP on the remaining variables is solved. Danna et al. (2005) considered an objective value cutoff constraint to force the solution of the sub-MILP to be better than the current best integer feasible solution. However, in our implementation within the context of LP/NLP-BB algorithm, there is no need for such a constraint, as constraint (4.29) on problem MP assures that the sub- 
MILP yields a lower objective value than the current upper bound. Table (4.3) illustrates how the RINS method is applied within the LP/NLP-BB framework in this research.

Table 4.3. Relaxation Induced Neighborhood Search (RINS) within LP/NLP-BB algorithm $y_{j}^{*}, z_{j}^{*}, x_{i j r}^{*}:=$ Best integer feasible solution at the current node;

$\hat{y}_{j}, \hat{z}_{j}, \hat{x}_{i j r}:=$ Solution of the LPR-MP problem at the current node;

$y_{j}^{L}, z_{j}^{L}, x_{i j r}^{L}:=$ Lower bounds of the variables $\& y_{j}^{U}, z_{j}^{U}, x_{i j r}^{U}:=$ Upper bounds of the variables;

Step 1: Add the following constraints to Problem (MP):

$$
\begin{array}{ll}
\text { - } \quad y_{y_{f}}^{L}:=y_{j}^{*} ; y_{y_{f}}^{U}:=y_{j}^{*} \quad \text { where: } y_{f}=\left\{j \mid \hat{y}_{j}=y_{j}^{*}\right\} \\
\text { - } \quad z_{z_{f}}^{L}:=z_{j}^{*} ; z_{z_{f}}^{U}:=z_{j}^{*} \quad \text { where: } z_{f}=\left\{j \mid \hat{z}_{j}=z_{j}^{*}\right\} \\
\text { - } \quad x_{x_{f}}^{L}:=x_{i j r}^{*} ; x_{x_{f}}^{U}:=x_{i j r}^{*} \quad \text { where: } x_{f}=\left\{(i, j, r) \mid \hat{x}_{i j r}=x_{i j r}^{*}\right\}
\end{array}
$$

Step 2: The resultant problem in step 1 is the Sub-MILP within RINS framework. Solve the SubMILP problem and report the solutions.

\subsubsection{Branching and node selection strategies}

Two strategic decisions that need to be made in a branch and bound framework are: variable selection for branching and selection of a node, from the remaining open nodes, that should be solved next in the algorithm. These two strategic decisions can be simply referred to as branching and node selection rules. There are many articles in the literature (e.g. Linderoth and Savelsbergh, 1999) that discuss and compare different approaches such as maximum fractionality, strong branching, and pseudocost-based method for branching; and best bound, depth first, best estimate, and adaptive (hybrid) methods for node selection rules. Although the pseudocost-based 
method has been used for branching in many different problems in the literature, and has been often stated as the most efficient method, a branching scheme that is similar to the one used by Daskin et. al. (2002) is utilized in this research, as it exploits the nature of the location problem. Based on this branching scheme, the first fractional location variable $\left(y_{j}\right)$ in the list of candidate locations is being branched, and if there is no $y_{j}$ taking a fractional value in the solution of a node, the fortification variables $\left(z_{j}\right)$ are considered for branching, followed by assignment variables

$\left(x_{i j r}\right)$. Furthermore, the best bound method is used for the node selection rule to faster improve the lower bound of the problem. The best bound method selects the node with best (lowest) bound. It should be noted that the solution of the LPR-MP problem at each node of the branch and bound yields a lower bound for the problem at that node, which is often referred as (lower) bound of the node. The minimum value of these lower bounds at open nodes can actually be considered as the lower bound of the primary problem, as there is no better solution possible for the problem. Thus, best bound method selects the node corresponding to the lower bound of the primary problem to be solved next in the algorithm, which results in improving the lower bound.

\subsubsection{Linearization generation at fractional solutions}

As mentioned earlier, Abhishek et al. (2010) improved the original LP/NLP-BB algorithm proposed by Quesada and Grossmann (1992) by considering linearization generation at fractional solution of the LP relaxation problem. Following their work, two different ways of linearization generation at fractional solutions to the LPR-MP problem are considered in this research. The first one simply linearizes the nonlinear constraint (4.7) in problem (P2) about the fractional solutions obtained for the LPR-MP problem. This way of linearization generation has some similarity to the Extended Cutting Plane (ECP) method proposed by Westerlund and Pettersson (1995), and for this 
reason it was called by Abhishek et al. (2010) as the ECP-based method. In the second method, first the NLP sub problem is solved at the fractional solutions obtained at LPR-MP problem, and then the nonlinear constraint (4.7) in problem (P2) is linearized about the solutions to the NLP sub problem. The second approach is similar to the original way of linearization cut generation on the LP/NLP-BB algorithm, except that here integer values can be fixed at fractional values before solving the NLP sub problem. Abhishek et al. (2010) called the second method as the fixfrac method. Like Abhishek et al. (2010), a combination of these methods is considered for linearization generation in this research. First ECP-based linearizations are generated until it appears that new additions are no longer useful, then the fixfrac method is used to generate a second set of linearization cuts until their addition is also stopped, and the algorithm goes to the primal heuristic section. To check whether the linearization generation using either the ECP-based or the fixfrac method should be stopped, the algorithm compares the objective value of LPR-MP problem at the current node (bound of the current node) before and after the linearization generation. If the bound of the current node has not been improved sufficiently, the linearization generation is stopped and the algorithm moves on to the next step.

The next section describes the details of how and when to generate the two types of linearization cuts discussed above.

\subsubsection{Cut management}

Encountering fractional solutions in a branch and bound framework, a fundamental implementation decision needs to be made on whether the algorithm should proceed by branching or by cutting. As indicated by Forrest et al. (1974), branching on a variable that does not significantly impact the solution of the LP relaxation problem at the consecutive nodes, results in 
a significant increase in the amount of work that needs to be done to completely process a node. Hence, the branching decisions made at the top of the branch and bound tree are the most critical ones by the very nature of the branch and bound methodology. This is especially important for the LP/NLP-BB algorithm, as in the original algorithm proposed by Quesada and Grossmann (1992), there are usually a few linearizations generated at the integer solutions and included in the LP relaxation master problem (LPR-MP) at the top of the branch and bound tree. Therefore, the LP relaxation master problem can be a naive approximation of the primary problem (MINLP), and branching decisions made based on this LP relaxation master problem may be very poor. The improvement by Abhishek et al. (2010) resolved this issue by generating linearization at fractional solutions and obtaining better linearization information early in the branch and bound process.

It should also be pointed out that the addition of linearization cuts enlarges the size of the LP relaxation problem, and thus it increases the solution time of the LP problem solved at each node. Therefore, there should be a tradeoff between cut generation and branching. There have been variety of cut management techniques in the literature to address this issue (e.g. Atamturk and Savelsbergh, 2005). Abhishek et al. (2010) considered a cut management technique that adds multiple rounds of linearization cuts to the LP relaxation problem at a given node. This cut management approach is slightly changed in this research and it is exploited within the LP/NLPBB framework. Based on the approach, three parameters are used to manage the addition of linearization cuts. The first one determines the likelihood of linearization generation at each node. This likelihood is biased in a way that nodes at the top of the search tree (closer to the root node) have a higher chance for linearization generation. The parameter is labeled as $\omega$, and the probability of generating a linearization at each node is defined by $\min \left\{\omega / 2^{d}, 1\right\}$ where $d$ corresponds to the level/depth of the node. Based on this likelihood function, there is lower chance 
of linearization generation for deeper nodes in the branch and bound (nodes with higher $d$ values). This is in accordance with what was mentioned earlier, that cutting should be prioritized over branching in the nodes on the top of the branch and bound. In addition, to avoid increasing the solution time of the LPR-MP problem, the chance of cut generation keeps decreasing as the algorithm proceeds to deeper nodes which are not as important as the nodes close to the root node of the branch and bound. In Abhishek et al. (2010) study, parameter $\omega$ for the ECP based method $\left(\omega_{\text {ecp }}\right)$ was considered as 10 , whereas this parameter for the fixfrac method $\left(\omega_{f f}\right)$ was set as 1 . The reason was to decrease the frequency of solving the nonlinear sub problem. This is not the case in the implementation of LP/NLP-BB algorithm for this research since the nonlinear sub problem can be trivially solved as explained in section (4.3.1). Therefore, $\omega_{f f}$ and $\omega_{e c p}$ were both set to 10 .

As mentioned in section (4.4.2.4), there is a need to check the efficiency of linearizations in improving the bound of the current node. Hence, the second parameter, labeled as $\zeta$, is used as a tolerance for the percentage change in the objective value of the LPR-MP problem. If this percentage is not at least $100 \times \zeta$, the algorithm proceeds by branching rather than adding more linearization cuts. The third parameter $\psi$ is considered to set a maximum number for linearization cut iterations (rounds) before branching. In other words, if none of the first two parameters stop the cutting rounds, the algorithm proceeds with branching after $\psi$ rounds of cut generations are performed at the current node of the search tree. Parameters $\zeta$ and $\psi$ are considered as 0.001 and 5 for both ECP based and fixfrac methods in this research.

Table (4.4) illustrates the pseudocode of the linearization generation algorithm along with the cut management technique used in this research. 
Table 4.4. Linearization generation at fractional solutions for the LPR-MP problem $\hat{y}_{j}, \hat{z}_{j}, \hat{t}_{j}, \hat{x}_{i j r}, \widehat{M}_{i j r}:=$ Fractional solution of the LPR-MP problem at the current node; rand $\in(0,1):=$ A uniformly generated random number at each node.

if rand $\leq \omega_{e c p} / 2^{d}$ AND $\left(M O F^{k}-M O F^{k-1}\right) / M O F^{k-1} \geq \zeta_{e c p}$ AND $n_{\text {ecp }} \leq \psi_{\text {ecp }}$ then Linearize nonlinear constraint (4.7) about the point $\hat{y}_{j}^{k}=\hat{y}_{j}, \hat{z}_{j}^{k}=\hat{z}_{j}, \hat{t}_{j}^{k}=\hat{t}_{j}, \hat{x}_{i j r}^{k}=\hat{x}_{i j r}$ and $\widehat{M}_{i j r}^{k}=\widehat{M}_{i j r}$ and add constraint (4.27) to the LPR-MP problem.

Set $n_{e c p}:=n_{e c p}+1$.

else if rand $\leq{ }^{\omega_{f f}} / 2^{d}$ AND $\left(M O F^{k}-M O F^{k-1}\right) / M O F^{k-1} \geq \zeta_{f f}$ AND $n_{f f} \leq \psi_{f f}$ then Let $\hat{y}_{j}^{k}=\hat{y}_{j}, \hat{z}_{j}^{k}=\hat{z}_{j}, \hat{x}_{i j r}^{k}=\hat{x}_{i j r}$ and $\widehat{M}_{i j r}^{k}=\widehat{M}_{i j r}$ and solve the NLP sub problem by evaluating the continuous variable $\hat{t}_{j}^{k}$ according to equation (4.21). Linearize the nonlinear constraint (4.7) about the solution of the NLP sub problem and add constraint (4.27) to the LPR-MP problem.

Set $n_{f f}:=n_{f f}+1$.

end if 


\section{Chapter 5 - Results and discussions}


In this chapter, three sets of computational experiments are presented to study the performance of the proposed solution methodologies (OA and LP/NLP-BB) presented in chapter 4 to solve problem $(\mathrm{P} 2)$. In the first set, the results obtained with the proposed algorithms are compared with those obtained from using available commercial solvers. For this purpose, a Branch-And-Reduce Optimization Navigator (BARON) solver within General Algebraic Modeling System (GAMS) is considered for comparison. BARON is a solver used to obtain the global solution of nonlinear (NLP) and mixed integer nonlinear programs (MINLP).

The second set of experiments is designed to evaluate and compare the computational performances of the OA and LP/NLP-BB algorithms in solving the problem on hand, under various sizes and parameter settings of the problem. Furthermore, some sensitivity analysis is performed on different parameters such as the weights associated to inventory and transportation costs ( $\theta$ and $\beta$ ), and fortification budget $(B)$, and managerial insights are derived. The third set of experiments is used to investigate the power and robustness of the LP/NLP-BB algorithm in solving larger, real-world level problem instances for different ranges of value of disruption parameter $(q)$, maximum level of backups $(R)$, fortification budget, and inventory and transportation weights. First, the dataset used and the computational setup are described in the following section.

\subsection{Experimental design}

As the model developed in this research combines three problems: a location-inventory, a facility disruption, and a facility fortification problem, the dataset used for the comparisons is adapted from different sources. The dataset sources considered in this research are from Daskin et. al. (2002), Snyder and Daskin (2005), Chen et. al. (2011) and Li et. al. (2013). All the above sources used the data provided by Daskin (1995), and readjusted it to their specific problems. The 
data of Daskin (1995) is derived from the 1990 census data, and mainly consists of three datasets: a 49-node set consisting of the state capitals of the contiguous United States plus Washington, D.C.; an 88-node set consisting of the 49-node set plus the 50 largest cities in the contiguous United States, excluding the capitals; and a 150-node set consisting of the 150 largest cities in the contiguous United States. Later on, Daskin (2013), in the second edition of his book, substituted the 150-node set with a 250-node set consisting of the 250 largest counties in the United States in 2010. In this research, the 49-node set, the 88-node set, and the upgraded version of the 150-node set, which consists of the seats of the 150 largest counties in the United States, are considered for the experiments. It should be noted that each of the nodes (cities) has a demand that needs to be satisfied, and it is also a candidate location for opening up a facility. Daskin et. al. (2002), obtained the demand by dividing the population data by 1000 and rounding the results to the nearest integer. Fixed facility location costs for the 49-node set and the 88-node set were obtained by dividing the median home value in the cities by 100 , whereas facility location costs were uniformly set to 100 for the 150-node set. According to Daskin et. al. (2002), the reason for these changes was to deal with smaller numbers. The transportation cost $\left(c_{i j}\right)$ is set to be proportional to the great circle distance between retailer $i$ and facility $j$. The great circle distance between two points is defined as the shortest distance between the points on the surface of a sphere. Table (5.1) illustrates the base values for other parameters used for all computational runs.

The tests were run using a laptop with a $2.6 \mathrm{GHz}$ i7-4720HQ CPU, the 64-bit version of the Windows 8 operating system, and an 8 GB RAM. Both of OA and LP/NLP-BB algorithms presented in chapter 4 were coded in GAMS, and CPLEX 12 was used to solve the MP and the LPR-MP master problem of the algorithms. 


\subsection{Computational experiment 1}

The aim of the first set of experiments is to compare the computational performance of the OA and LP/NLP-BB algorithms for solving model (P2) with the BARON solver to directly solve model (P1). Table (5.2) shows the computational times and objective function values obtained from all methods for six relatively small problem instances. The data for these six problems respectively corresponds to the 5, 10, 15, 20, 25 and 30 largest counties in the United States. For this set of computational runs, along with the base values for the parameters in Table (5.1), the fortification budget $(B)$ is set equal to 60 , and Weight factors associated with transportation and

Table 5.1. Base values for model parameters

\begin{tabular}{cc}
\hline Parameter & Value \\
\hline$A_{j}$ & 10 \\
$g_{j}$ & 10 \\
$d_{j}$ & 5 \\
$h_{j}$ & 1 \\
$R$ & 3 \\
$q$ & 0.05 \\
$\eta$ & 100 \\
$S_{j}$ & 30 \\
Max \# of Iterations for OA & 1000 \\
Max \# of branch and bound & 1000 \\
nodes for LP/NLP-BB & \\
\hline
\end{tabular}


inventory costs are considered as $\beta=0.001$ and $\theta=0.1$. Furthermore, the algorithms should be solved to an optimality gap of zero to be able to fairly compare the performance of the algorithms.

As explained in chapter 4, the OA master problem (MP) is not required to be solved to optimality. Therefore, the CLPEX solver used to solve the MP problem is stopped as soon as a feasible solution is obtained. In this case, the MP problem does not provide a valid lower bound. Hence, the optimality gap cannot be obtained for the OA method, and the algorithm is terminated once the MP problem becomes infeasible, or other termination criteria are met. The maximum solution time is set to 600 seconds for all methods, at which the algorithms are stopped and the final solutions are reported. The results in table (5.2) show that both OA and LP/NLP-BB algorithms are efficient in solving all problem instances, whereas BARON solver is not an efficient method for larger problem instances, like 20 nodes, and it does not even provide an optimal solution for 25-node or larger problems.

Table 5.2. Comparisons of BARON solver with $O A$ and LP/NLP-BB algorithms

\begin{tabular}{|c|c|c|c|c|c|c|}
\hline \multirow{2}{*}{$\begin{array}{c}\text { Problem } \\
\text { Instance }\end{array}$} & \multicolumn{3}{|c|}{ BARON } & \multicolumn{2}{c|}{ OA } & \multicolumn{2}{c|}{\begin{tabular}{c} 
LP/NLP-BB \\
\cline { 2 - 7 } \\
$($ Sec. $)$
\end{tabular}} & $\begin{array}{c}\text { Objective Value } \\
(\text { Gap\% })\end{array}$ & $\begin{array}{c}\text { Time } \\
(\text { Sec. })\end{array}$ & Objective Value & $\begin{array}{c}\text { Time } \\
(\text { Sec. })\end{array}$ & $\begin{array}{c}\text { Objective Value } \\
(\text { Gap\% })\end{array}$ \\
\hline 5-node & 0.11 & $1301.75(0)$ & 0.14 & 1301.75 & 0.08 & $1301.75(0)$ \\
\hline 10-node & 2.03 & $2266.24(0)$ & 1.05 & 2266.24 & 0.64 & $2266.24(0)$ \\
\hline 15-node & 15.03 & $3122.34(0)$ & 5.14 & 3122.34 & 3.47 & $3122.34(0)$ \\
\hline 20-node & 143.77 & $3685.73(0)$ & 10.74 & 3685.73 & 5.9 & $3685.73(0)$ \\
\hline 25-node & $>600$ & $4290.75(5.33)$ & 7.83 & 4287.77 & 7.58 & $4287.77(0)$ \\
\hline 30-node & $>600$ & $4733.58(8.05)$ & 16.52 & 4728.26 & 10.59 & $4728.26(0)$ \\
\hline
\end{tabular}




\subsection{Computational experiment 2}

In this section, LP/NLP-BB and OA algorithms are compared using large problem instances, 49-node and 88-node, for a variety of parameter settings. The OA algorithm could not solve the 150-node problems, so only the LP/NLP-BB method was used, as will be discussed in section (5.4). Tables (5.3)-(5.10) summarize the results obtained. Whenever the objective values obtained by the LP/NLP-BB and the OA methods are different, the lowest value is given in the tables in bold. Each table represents the results for a range of values for $\beta$ and $\theta$, weight factors associated with the inventory and transportation costs, and a specific value for the fortification budget $(B)$. The range of values for $\beta$ and $\theta$ are correspondent to Daskin et. al. (2002), and the values for $B$ are picked from $\mathrm{Li}$ et. al. (2013). In addition to the number of opened facilities, the number of fortified facilities and the cost of fortifications are shown in table (5.10), which has the highest budget for fortification. It should be noted that the optimality gap for the LP/NLP-BB algorithm is considered as $0.5 \%$ for all the problem instances in this section.

As it can be observed, the implementation of the LP/NLP-BB algorithm in this research significantly outperforms the OA method in all of these computational runs. While the LP/NLPBB algorithm is very efficient in all problem instances, the OA method has difficulty finding the optimal solution, especially when the inventory cost is dominating (when $\theta=5,10$, or 20 and $\beta=0.005)$ and the size of the problem is increased (88-node).

The tables also provide the number of opened facilities for each problem instance at the solution obtained by the LP/NLP-BB algorithm, as the solution of OA was either the same or worse than the LP/NLP-BB algorithm solution. In general, the number of opened facilities increases with the increase in $\beta$, and it decreases with the increase in $\theta$. This is not surprising since when the transportation cost is more prevailing, more facilities should be opened to manage this cost, and 
when the relative importance of inventory cost goes up, customer demand should be pooled to fewer facilities to avoid the increase in inventory cost associated with opening more facilities.

One interesting thing that is observed here is that the decrease in the number of open facilities in extreme cases of inventory $\operatorname{cost}($ e.g. $\theta=20$ and $\beta=0.005)$ is not as significant as it is in the study by Daskin et. al. (2002). The reason can be attributed to the fact that the model in this research incorporates a reliability fixed-charge location problem (RFLP) into a joint location inventory framework, and as mentioned by Snyder and Daskin (2005), the risk-diversification effect tends to increase the number of opened facilities. As mentioned in chapter 1, a joint location inventory framework is to obtain an optimal tradeoff between transportation, inventory, and facility setup costs. It is helpful to learn that the consideration of disruption in this framework can change the optimal tradeoff, and consequently change the strategic location decision.

The results also demonstrate that changing the fortification budget $(B)$ does not significantly influence the computational runs for both algorithms. From the managerial point of view, it can be seen that increasing the fortification budget $(B)$ slightly decreases the number of opened facilities and the optimal cost (objective value). The reason is that increasing the fortification budget results in more fortified facilities in the network, and thus lower backup level $(r)$ assignments which leads to a lower transportation cost. It should be pointed out that the increase for the fortification budget needs to be justified by the cost savings. For instance, solutions for 49node problem are the same for $B=240, B=300$ and $B=360$, which means that further increasing the budget is not beneficial. 


\subsection{Computational experiment 3}

This section includes the numerical results for the 150-node datasets solved by the LP/NLP-BB algorithm. As the OA algorithm was not efficient for the smaller problem instances, it was not considered for the 150-node computational runs. An optimality gap of $1 \%$ is considered as the termination criterion for the LP/NLP-BB algorithm used for all the problem instances in this section.

Tables (5.11.a-d) summarize the results for the base case $(q=0.05, R=3)$, and tables (5.12)-(5.14) and tables (5.15)-(5.16) respectively illustrate the sensitivity analysis results for the maximum number of backup levels $(R=2, R=4, R=5)$ and disruption parameter $(q=$ $0.01, q=0.03$ ). All of these tables include the breakdown (in percentage) of the total cost into its components (Fixed Set-up, Inventory, Shipment, and Penalty costs) at the solution obtained for each problem instance. The results show that the performance of the developed solution approach based on LP/NLP-BB is robust for a broad range of parameters, and despite the increased problem sizes, it can still solve most of these instances efficiently to less than $1 \%$ optimality gap.

In general, increasing the fortification budget $(B)$ or the maximum number of backup levels $(R)$, or decreasing the chance of disruption $(q)$, result in a lower total cost. Moreover, the number of opened facilities slightly increases with the increase in $q$, and it slightly decreases with the increase in $B$. Furthermore, same as the results for the 49-node and 88 -node instances, the number of opened facilities decreases whenever the inventory cost has a higher contribution in the total cost, and it increases when the transportation cost is more dominant.

Finally the last set of results, table (5.17), is to demonstrate the advantages obtained by planning for disruption. For this purpose, a nominal cost is obtained (by assuming that all the facilities are reliable (non-failable)) and it is compared with the following two expected costs when 
failures are considered: Cost with no planning, and cost with planning. The nominal cost can be obtained by fixing the variables $z_{j}$ to 1 , removing constraint sets (4.11) and (4.12), considering $R=1$ (No backups) for problem (P2), and solving the resulting problem. The expected cost with no planning is obtained by modifying the solution for the nominal cost and considering that the opened facilities can be disrupted with a chance of $q=0.001$. In other words, each opened facility can be failed, and consequently, the customers assigned to the failed facility will be assigned to the emergency facility, resulting in the addition of a penalty cost to the system. The expected cost with planning is obtained using the disruption planning framework developed in this research; considering $R=3$ backup levels and a fortification budget $(B)$ of 180, and solving problem (P2). The expected cost with planning is then obtained by adding the fortification budget $(B=180)$ to the value of the objective function obtained from the solution of problem (P2). The results show that, even when the chance of disruption is very low $(q=0.001)$, with little additional investment (less than $3 \%$ of the nominal cost), a dramatic increase (more than 200\%) in the total cost can be avoided, and the system can be made significantly more resilient to disruptions. 
Table 5.3. Comparisons of $O A$ and $L P / N L P-B B$ algorithms with $B=0$

\begin{tabular}{|c|c|c|c|c|c|c|c|}
\hline \multicolumn{3}{|c|}{ Problem Instance } & \multirow{2}{*}{$\begin{array}{c}\# \\
\text { Opened } \\
\text { facilities }\end{array}$} & \multicolumn{2}{|c|}{ LP/NLP-BB } & \multicolumn{2}{|c|}{$\mathrm{OA}$} \\
\hline Size & $\beta$ & $\theta$ & & $\begin{array}{l}\text { Time } \\
(\mathrm{Sec} .)\end{array}$ & $\begin{array}{c}\text { Objective } \\
\text { Value }\end{array}$ & $\begin{array}{l}\text { Time } \\
(\mathrm{Sec} .)\end{array}$ & $\begin{array}{c}\text { Objective } \\
\text { Value }\end{array}$ \\
\hline 49-node & 0.001 & 0.1 & 4 & 1.34 & 5903.61 & 11.66 & 5903.15 \\
\hline 49-node & 0.002 & 0.1 & 6 & 0.66 & 9005.29 & 14.55 & 9005.29 \\
\hline 49-node & 0.003 & 0.1 & 7 & 0.92 & 11159.78 & 7.17 & 11159.78 \\
\hline 49-node & 0.004 & 0.1 & 10 & 0.52 & 13225.12 & 7.33 & 13225.12 \\
\hline 49-node & 0.005 & 0.1 & 11 & 0.41 & 14679.16 & 8.91 & 14679.16 \\
\hline 49-node & 0.002 & 0.2 & 5 & 1.56 & 9111.51 & 22.53 & 9111.51 \\
\hline 49-node & 0.005 & 0.5 & 11 & 0.19 & 15383.66 & 12.38 & 15383.66 \\
\hline 49-node & 0.005 & 1 & 11 & 0.36 & 15815.82 & 31.41 & 15815.82 \\
\hline 49-node & 0.005 & 5 & 10 & 0.52 & 17604.05 & 281.29 & 17604.05 \\
\hline 49-node & 0.005 & 10 & 9 & 0.72 & 18849.5 & 474.72 & 18849.5 \\
\hline 49-node & 0.005 & 20 & 8 & 0.87 & 20571.28 & 213.63 & 20571.28 \\
\hline 88-node & 0.001 & 0.1 & 9 & 2.59 & 14378.67 & 83.142 & 14378.21 \\
\hline 88-node & 0.002 & 0.1 & 12 & 0.78 & 21489.43 & 32.874 & 21489.43 \\
\hline 88-node & 0.003 & 0.1 & 17 & 0.97 & 26439.37 & 32.717 & 26439.37 \\
\hline 88-node & 0.004 & 0.1 & 22 & 0.92 & 30781.86 & 22.393 & 30781.86 \\
\hline 88-node & 0.005 & 0.1 & 24 & 0.91 & 33204.51 & 20.079 & 33204.51 \\
\hline 88-node & 0.002 & 0.2 & 13 & 1.85 & 22314.69 & 110.391 & 22314.69 \\
\hline 88-node & 0.005 & 0.5 & 24 & 0.66 & 34917.35 & 81.124 & 34917.35 \\
\hline 88-node & 0.005 & 1 & 24 & 0.95 & 36200.81 & 216.063 & 36200.81 \\
\hline 88-node & 0.005 & 5 & 22 & 1.08 & 41617.28 & $>600$ & 41931.53 \\
\hline 88-node & 0.005 & 10 & 19 & 1.41 & 45675.94 & $>600$ & 45781.02 \\
\hline 88-node & 0.005 & 20 & 17 & 1.77 & 51415.76 & $>600$ & 51849.01 \\
\hline
\end{tabular}


Table 5.4. Comparisons of $O A$ and $L P / N L P-B B$ algorithms with $B=30$

\begin{tabular}{|c|c|c|c|c|c|c|c|}
\hline \multicolumn{2}{|c|}{ Problem Instance } & \multirow{2}{*}{$\begin{array}{c}\# \\
\text { Opened }\end{array}$} & \multicolumn{2}{c|}{ LP/NLP-BB } & \multicolumn{2}{c|}{ OA } \\
\cline { 6 - 8 } fize & $\beta$ & $\theta$ & facilities & $\begin{array}{c}\text { Time } \\
\text { (Sec.) }\end{array}$ & $\begin{array}{c}\text { Objective } \\
\text { Value }\end{array}$ & $\begin{array}{c}\text { Time } \\
\text { (Sec.) }\end{array}$ & $\begin{array}{c}\text { Objective } \\
\text { Value }\end{array}$ \\
\hline 49-node & 0.001 & 0.1 & 4 & 30.50 & 5793.12 & 31.89 & 5793.12 \\
\hline 49-node & 0.002 & 0.1 & 5 & 44.60 & 8794.07 & 15.61 & 8794.07 \\
\hline 49-node & 0.003 & 0.1 & 7 & 0.97 & 11099.3 & 14.58 & 11099.3 \\
\hline 49-node & 0.004 & 0.1 & 9 & 1.25 & 13093.17 & 7.30 & 13093.17 \\
\hline 49-node & 0.005 & 0.1 & 11 & 1.28 & 14636.58 & 11.66 & 14636.58 \\
\hline 49-node & 0.002 & 0.2 & 5 & 2.53 & 8926.31 & 24.75 & 8926.31 \\
\hline 49-node & 0.005 & 0.5 & 11 & 1.16 & 15212.91 & 23.47 & 15212.91 \\
\hline 49-node & 0.005 & 1 & 11 & 1.30 & 15644.78 & 57.70 & 15644.78 \\
\hline 49-node & 0.005 & 5 & 10 & 1.45 & $\mathbf{1 7 4 2 6 . 1 1}$ & $>600$ & 17563.52 \\
\hline 49-node & 0.005 & 10 & 9 & 2.06 & $\mathbf{1 8 5 7 9 . 6 9}$ & $>600$ & 18621.45 \\
\hline 49-node & 0.005 & 20 & 8 & 2.11 & $\mathbf{2 0 2 5 4 . 8 1}$ & $>600$ & 20387.12 \\
\hline 88-node & 0.001 & 0.1 & 9 & 21.72 & 13918.27 & 163.67 & 13918.09 \\
\hline 88-node & 0.002 & 0.1 & 11 & 8.63 & 20982.97 & 70.389 & 20982.97 \\
\hline 88-node & 0.003 & 0.1 & 16 & 1.59 & 26510.36 & 81.471 & 26510.36 \\
\hline 88-node & 0.004 & 0.1 & 22 & 9.39 & 29990.18 & 75.609 & 29990.18 \\
\hline 88-node & 0.005 & 0.1 & 24 & 7.33 & 32846.51 & 53.984 & 32846.51 \\
\hline 88-node & 0.002 & 0.2 & 11 & 11.76 & 21159.72 & 132.029 & 21159.72 \\
\hline 88-node & 0.005 & 0.5 & 24 & 7.73 & 34557.45 & 191.328 & 34557.45 \\
\hline 88-node & 0.005 & 1 & 24 & 7.97 & $\mathbf{3 5 8 3 9 . 4 9}$ & $>600$ & 36164.68 \\
\hline 88-node & 0.005 & 5 & 22 & 8.63 & $\mathbf{4 1 2 4 9 . 9 5}$ & $>600$ & 41597.64 \\
\hline 88-node & 0.005 & 10 & 19 & 8.80 & $\mathbf{4 5 3 0 4 . 1 2}$ & $>600$ & 45785.83 \\
\hline 88-node & 0.005 & 20 & 16 & 9.89 & $\mathbf{5 1 0 3 7 . 5 8}$ & $>600$ & 51866.56 \\
\hline
\end{tabular}


Table 5.5. Comparisons of $O A$ and $L P / N L P-B B$ algorithms with $B=60$

\begin{tabular}{|c|c|c|c|c|c|c|c|}
\hline \multicolumn{2}{|c|}{ Problem Instance } & \multirow{2}{*}{$\begin{array}{c}\# \\
\text { Opened }\end{array}$} & \multicolumn{2}{c|}{ LP/NLP-BB } & \multicolumn{2}{c|}{ OA } \\
Size & $\beta$ & $\theta$ & facilities & $\begin{array}{c}\text { Time } \\
(\text { Sec. })\end{array}$ & $\begin{array}{c}\text { Objective } \\
\text { Value }\end{array}$ & $\begin{array}{c}\text { Time } \\
\text { (Sec.) }\end{array}$ & $\begin{array}{c}\text { Objective } \\
\text { Value }\end{array}$ \\
\hline 49-node & 0.001 & 0.1 & 4 & 2.75 & 5652.91 & 19.61 & 5652.91 \\
\hline 49-node & 0.002 & 0.1 & 5 & 1.73 & 8638.44 & 12.72 & 8638.44 \\
\hline 49-node & 0.003 & 0.1 & 8 & 1.09 & 10995.91 & 16.56 & 10995.91 \\
\hline 49-node & 0.004 & 0.1 & 9 & 1.00 & 12925.33 & 10.24 & 12925.33 \\
\hline 49-node & 0.005 & 0.1 & 11 & 0.84 & 14453.6 & 11.80 & 14453.6 \\
\hline 49-node & 0.002 & 0.2 & 5 & 1.61 & 8771.29 & 12.91 & 8771.29 \\
\hline 49-node & 0.005 & 0.5 & 11 & 0.81 & 15029.51 & 20.53 & 15029.51 \\
\hline 49-node & 0.005 & 1 & 11 & 0.95 & 15461.06 & 30.31 & 15461.06 \\
\hline 49-node & 0.005 & 5 & 9 & 1.13 & 17247.03 & 414.65 & 17247.03 \\
\hline 49-node & 0.005 & 10 & 9 & 1.80 & $\mathbf{1 8 4 1 0 . 2 9}$ & $>600$ & 18469.41 \\
\hline 49-node & 0.005 & 20 & 8 & 2.05 & $\mathbf{2 0 0 9 1 . 4}$ & $>600$ & 20116.64 \\
\hline 88-node & 0.001 & 0.1 & 9 & 16.69 & 13667.96 & 116.64 & 13667.96 \\
\hline 88-node & 0.002 & 0.1 & 11 & 2.19 & 20704.95 & 37.921 & 20704.95 \\
\hline 88-node & 0.003 & 0.1 & 16 & 7.92 & 26175.89 & 130.624 & 26175.89 \\
\hline 88-node & 0.004 & 0.1 & 21 & 6.33 & 29868.58 & 57.014 & 29868.58 \\
\hline 88-node & 0.005 & 0.1 & 24 & 5.58 & 32756.23 & 44.922 & 32756.23 \\
\hline 88-node & 0.002 & 0.2 & 11 & 7.01 & 20836.9 & 139.873 & 20836.9 \\
\hline 88-node & 0.005 & 0.5 & 24 & 5.56 & 34462.3 & 161.063 & 34462.3 \\
\hline 88-node & 0.005 & 1 & 23 & 5.39 & 35740.7 & 556.205 & 35740.7 \\
\hline 88-node & 0.005 & 5 & 22 & 6.25 & $\mathbf{4 0 1 3 5 . 7 9}$ & $>600$ & 40861.89 \\
\hline 88-node & 0.005 & 10 & 19 & 6.69 & $\mathbf{4 5 1 7 8 . 4 3}$ & $>600$ & 45736.65 \\
\hline 88-node & 0.005 & 20 & 16 & 7.55 & $\mathbf{4 9 1 9 5 . 5 9}$ & $>600$ & 50042.63 \\
\hline
\end{tabular}


Table 5.6. Comparisons of $O A$ and LP/NLP-BB algorithms with $B=120$

\begin{tabular}{|c|c|c|c|c|c|c|c|}
\hline \multicolumn{3}{|c|}{ Problem Instance } & \multirow{2}{*}{$\begin{array}{c}\# \\
\text { Opened } \\
\text { facilities }\end{array}$} & \multicolumn{2}{|c|}{ LP/NLP-BB } & \multicolumn{2}{|c|}{ OA } \\
\hline Size & $\beta$ & $\theta$ & & $\begin{array}{l}\text { Time } \\
\text { (Sec.) }\end{array}$ & $\begin{array}{c}\text { Objective } \\
\text { Value }\end{array}$ & $\begin{array}{l}\text { Time } \\
\text { (Sec.) }\end{array}$ & $\begin{array}{c}\text { Objective } \\
\text { Value }\end{array}$ \\
\hline 49-node & 0.001 & 0.1 & 4 & 1.11 & 5569.56 & 4.45 & 5569.56 \\
\hline 49-node & 0.002 & 0.1 & 5 & 1.25 & 8492.44 & 4.36 & 8492.44 \\
\hline 49-node & 0.003 & 0.1 & 8 & 0.99 & 10807.11 & 6.17 & 10807.11 \\
\hline 49-node & 0.004 & 0.1 & 8 & 0.83 & 12723.46 & 7.74 & 12723.46 \\
\hline 49-node & 0.005 & 0.1 & 11 & 0.84 & 14304.14 & 15.45 & 14304.14 \\
\hline 49-node & 0.002 & 0.2 & 5 & 1.28 & 8623.65 & 11.17 & 8623.65 \\
\hline 49-node & 0.005 & 0.5 & 11 & 0.95 & 14880.44 & 40.81 & 14880.44 \\
\hline 49-node & 0.005 & 1 & 11 & 1.03 & 15312.28 & 76.25 & 15312.28 \\
\hline 49-node & 0.005 & 5 & 9 & 1.30 & 16981.84 & 122.75 & 16981.84 \\
\hline 49-node & 0.005 & 10 & 8 & 1.63 & 18140.14 & 347.89 & 18140.14 \\
\hline 49-node & 0.005 & 20 & 8 & 1.89 & 19778.67 & $>600$ & 19848.34 \\
\hline 88-node & 0.001 & 0.1 & 9 & 12.33 & 13386.35 & 61.219 & 13386.35 \\
\hline 88-node & 0.002 & 0.1 & 11 & 2.08 & 20407.34 & 30.998 & 20407.34 \\
\hline 88-node & 0.003 & 0.1 & 16 & 3.84 & 25878.14 & 105.453 & 25878.14 \\
\hline 88-node & 0.004 & 0.1 & 21 & 3.39 & 29569.27 & 61.483 & 29569.27 \\
\hline 88-node & 0.005 & 0.1 & 24 & 2.81 & 32398.18 & 31.03 & 32398.18 \\
\hline 88-node & 0.002 & 0.2 & 11 & 4.94 & 20618.64 & 139.327 & 20618.64 \\
\hline 88-node & 0.005 & 0.5 & 23 & 2.72 & 34103.09 & 122.217 & 34103.09 \\
\hline 88-node & 0.005 & 1 & 22 & 2.81 & 35380.6 & 423.388 & 35380.6 \\
\hline 88-node & 0.005 & 5 & 21 & 4.05 & 39871.99 & $>600$ & 40400.74 \\
\hline 88-node & 0.005 & 10 & 19 & 3.88 & 44811.86 & $>600$ & 45543.96 \\
\hline 88-node & 0.005 & 20 & 16 & 4.97 & 48625.1 & $>600$ & 49188.74 \\
\hline
\end{tabular}


Table 5.7. Comparisons of $O A$ and LP/NLP-BB algorithms with $B=180$

\begin{tabular}{|c|c|c|c|c|c|c|c|}
\hline \multicolumn{3}{|c|}{ Problem Instance } & \multirow{2}{*}{$\begin{array}{c}\# \\
\text { Opened } \\
\text { facilities }\end{array}$} & \multicolumn{2}{|c|}{ LP/NLP-BB } & \multicolumn{2}{|c|}{$\mathrm{OA}$} \\
\hline Size & $\beta$ & $\theta$ & & $\begin{array}{l}\text { Time } \\
\text { (Sec.) }\end{array}$ & $\begin{array}{c}\text { Objective } \\
\text { Value }\end{array}$ & $\begin{array}{l}\text { Time } \\
\text { (Sec.) }\end{array}$ & $\begin{array}{c}\text { Objective } \\
\text { Value }\end{array}$ \\
\hline 49-node & 0.001 & 0.1 & 4 & 1.06 & 5569.56 & 4.31 & 5569.56 \\
\hline 49-node & 0.002 & 0.1 & 5 & 0.80 & 8455.71 & 2.80 & 8455.71 \\
\hline 49-node & 0.003 & 0.1 & 8 & 0.80 & 10705.44 & 3.41 & 10705.44 \\
\hline 49-node & 0.004 & 0.1 & 8 & 0.78 & 12594.61 & 7.64 & 12594.61 \\
\hline 49-node & 0.005 & 0.1 & 11 & 0.69 & 14159.15 & 7.58 & 14159.15 \\
\hline 49-node & 0.002 & 0.2 & 5 & 0.77 & 8587.08 & 4.45 & 8587.08 \\
\hline 49-node & 0.005 & 0.5 & 11 & 0.81 & 14735.36 & 14.63 & 14735.36 \\
\hline 49-node & 0.005 & 1 & 11 & 0.92 & 15167.14 & 30.22 & 15167.14 \\
\hline 49-node & 0.005 & 5 & 9 & 1.12 & 16821.94 & 79.83 & 16821.94 \\
\hline 49-node & 0.005 & 10 & 8 & 1.36 & 17980.44 & 120.63 & 17980.44 \\
\hline 49-node & 0.005 & 20 & 8 & 1.55 & 19618.81 & 480.15 & 19618.81 \\
\hline 88-node & 0.001 & 0.1 & 9 & 10.95 & 13213.09 & 62.671 & 13213.09 \\
\hline 88-node & 0.002 & 0.1 & 11 & 2.00 & 20245.46 & 43.89 & 20245.46 \\
\hline 88-node & 0.003 & 0.1 & 16 & 3.98 & 25699.12 & 63.248 & 25699.12 \\
\hline 88-node & 0.004 & 0.1 & 21 & 3.23 & 29354.74 & 63.797 & 29354.74 \\
\hline 88-node & 0.005 & 0.1 & 24 & 3.28 & 32137.71 & 32.484 & 32137.71 \\
\hline 88-node & 0.002 & 0.2 & 11 & 5.33 & 20475.86 & 128.935 & 20475.86 \\
\hline 88-node & 0.005 & 0.5 & 23 & 3.25 & 33843.08 & 103.784 & 33843.08 \\
\hline 88-node & 0.005 & 1 & 22 & 3.22 & 35120.94 & 460.067 & 35120.94 \\
\hline 88-node & 0.005 & 5 & 20 & 4.00 & 39413.79 & $>600$ & 40169.17 \\
\hline 88-node & 0.005 & 10 & 18 & 4.52 & 44454.76 & $>600$ & 45138.26 \\
\hline 88-node & 0.005 & 20 & 16 & 5.72 & 48069.54 & $>600$ & 49040.98 \\
\hline
\end{tabular}


Table 5.8. Comparisons of OA and LP/NLP-BB algorithms with B=240

\begin{tabular}{|c|c|c|c|c|c|c|c|}
\hline \multicolumn{3}{|c|}{ Problem Instance } & \multirow{2}{*}{$\begin{array}{c}\# \\
\text { Opened } \\
\text { facilities }\end{array}$} & \multicolumn{2}{|c|}{ LP/NLP-BB } & \multicolumn{2}{|c|}{$\mathrm{OA}$} \\
\hline Size & $\beta$ & $\theta$ & & $\begin{array}{l}\text { Time } \\
(\text { Sec.) }\end{array}$ & $\begin{array}{c}\text { Objective } \\
\text { Value }\end{array}$ & $\begin{array}{l}\text { Time } \\
\text { (Sec.) }\end{array}$ & $\begin{array}{c}\text { Objective } \\
\text { Value }\end{array}$ \\
\hline 49-node & 0.001 & 0.1 & 4 & 1.14 & 5569.56 & 4.39 & 5569.56 \\
\hline 49-node & 0.002 & 0.1 & 5 & 0.78 & 8455.71 & 2.67 & 8455.71 \\
\hline 49-node & 0.003 & 0.1 & 8 & 0.61 & 10689.97 & 3.98 & 10689.97 \\
\hline 49-node & 0.004 & 0.1 & 8 & 0.69 & 12500.04 & 3.03 & 12500.04 \\
\hline 49-node & 0.005 & 0.1 & 11 & 0.77 & 13917.04 & 5.25 & 13917.04 \\
\hline 49-node & 0.002 & 0.2 & 5 & 0.73 & 8587.08 & 4.44 & 8587.08 \\
\hline 49-node & 0.005 & 0.5 & 11 & 0.80 & 14492.96 & 9.52 & 14492.96 \\
\hline 49-node & 0.005 & 1 & 11 & 0.86 & 14924.51 & 25.50 & 14924.51 \\
\hline 49-node & 0.005 & 5 & 9 & 1.02 & 16677.22 & 27.97 & 16677.22 \\
\hline 49-node & 0.005 & 10 & 8 & 1.08 & 17866.32 & 29.16 & 17866.32 \\
\hline 49-node & 0.005 & 20 & 8 & 1.30 & 19506.61 & 69.77 & 19506.61 \\
\hline 88-node & 0.001 & 0.1 & 9 & 9.85 & 13139.88 & 48.108 & 13139.88 \\
\hline 88-node & 0.002 & 0.1 & 10 & 6.92 & 20382.52 & 48.485 & 20382.52 \\
\hline 88-node & 0.003 & 0.1 & 15 & 1.59 & 25614.55 & 70.642 & 25614.55 \\
\hline 88-node & 0.004 & 0.1 & 21 & 3.92 & 29196.78 & 47.11 & 29196.78 \\
\hline 88-node & 0.005 & 0.1 & 23 & 2.70 & 31947.44 & 100.361 & 31947.44 \\
\hline 88-node & 0.002 & 0.2 & 10 & 5.73 & 20359.59 & 39.282 & 20359.59 \\
\hline 88-node & 0.005 & 0.5 & 23 & 2.44 & 33652.55 & 126.377 & 33652.55 \\
\hline 88-node & 0.005 & 1 & 22 & 2.44 & 34930.23 & 282.404 & 34930.23 \\
\hline 88-node & 0.005 & 5 & 20 & 2.91 & 38922.26 & $>600$ & 39527.43 \\
\hline 88-node & 0.005 & 10 & 18 & 3.38 & 43862.62 & $>600$ & 44562.64 \\
\hline 88-node & 0.005 & 20 & 15 & 4.50 & 47376.55 & $>600$ & 48071.87 \\
\hline
\end{tabular}


Table 5.9. Comparisons of OA and LP/NLP-BB algorithms with $B=300$

\begin{tabular}{|c|c|c|c|c|c|c|c|}
\hline \multicolumn{3}{|c|}{ Problem Instance } & \multirow{2}{*}{$\begin{array}{c}\# \\
\text { Opened } \\
\text { facilities }\end{array}$} & \multicolumn{2}{|c|}{ LP/NLP-BB } & \multicolumn{2}{|c|}{$\mathrm{OA}$} \\
\hline Size & $\beta$ & $\theta$ & & $\begin{array}{l}\text { Time } \\
(\mathrm{Sec} .)\end{array}$ & $\begin{array}{c}\text { Objective } \\
\text { Value }\end{array}$ & $\begin{array}{c}\text { Time } \\
(\mathrm{Sec} .)\end{array}$ & $\begin{array}{c}\text { Objective } \\
\text { Value }\end{array}$ \\
\hline 49-node & 0.001 & 0.1 & 4 & 1.09 & 5569.56 & 4.42 & 5569.56 \\
\hline 49-node & 0.002 & 0.1 & 5 & 0.75 & 8455.71 & 2.72 & 8455.71 \\
\hline 49-node & 0.003 & 0.1 & 8 & 0.59 & 10689.97 & 3.97 & 10689.97 \\
\hline 49-node & 0.004 & 0.1 & 8 & 0.61 & 12500.04 & 3.10 & 12500.04 \\
\hline 49-node & 0.005 & 0.1 & 11 & 0.59 & 13917.04 & 3.14 & 13917.04 \\
\hline 49-node & 0.002 & 0.2 & 5 & 0.81 & 8587.08 & 4.53 & 8587.08 \\
\hline 49-node & 0.005 & 0.5 & 11 & 0.67 & 14492.96 & 5.36 & 14492.96 \\
\hline 49-node & 0.005 & 1 & 11 & 0.76 & 14924.51 & 8.17 & 14924.51 \\
\hline 49-node & 0.005 & 5 & 9 & 0.86 & 16677.22 & 42.19 & 16677.22 \\
\hline 49-node & 0.005 & 10 & 8 & 0.97 & 17866.32 & 21.86 & 17866.32 \\
\hline 49-node & 0.005 & 20 & 8 & 1.00 & 19506.61 & 45.61 & 19506.61 \\
\hline 88-node & 0.001 & 0.1 & 9 & 4.25 & 13107.92 & 19.985 & 13107.92 \\
\hline 88-node & 0.002 & 0.1 & 10 & 1.42 & 19870.21 & 27.75 & 19870.21 \\
\hline 88-node & 0.003 & 0.1 & 15 & 3.72 & 25396.28 & 32.374 & 25396.28 \\
\hline 88-node & 0.004 & 0.1 & 21 & 2.89 & 29056.63 & 52.562 & 29056.63 \\
\hline 88-node & 0.005 & 0.1 & 23 & 2.52 & 31876.01 & 55.547 & 31876.01 \\
\hline 88-node & 0.002 & 0.2 & 10 & 3.20 & 20241.29 & 28.626 & 20241.29 \\
\hline 88-node & 0.005 & 0.5 & 23 & 2.63 & 33513.43 & 150.375 & 33513.43 \\
\hline 88-node & 0.005 & 1 & 22 & 2.56 & 34739.25 & 195.077 & 34739.25 \\
\hline 88-node & 0.005 & 5 & 20 & 3.06 & 38692.38 & $>600$ & 39111.57 \\
\hline 88-node & 0.005 & 10 & 18 & 3.70 & 43533.06 & $>600$ & 44115.93 \\
\hline 88-node & 0.005 & 20 & 15 & 4.58 & 47047.46 & $>600$ & 47882.98 \\
\hline
\end{tabular}


Table 5.10. Comparisons of $O A$ and $L P / N L P-B B$ algorithms with $B=360$

\begin{tabular}{|c|c|c|c|c|c|c|c|c|c|}
\hline \multicolumn{3}{|c|}{ Problem Instance } & \multirow{2}{*}{$\begin{array}{c}\# \\
\text { Opened } \\
\text { facilities }\end{array}$} & \multirow{2}{*}{$\begin{array}{c}\text { \# } \\
\text { Fortified } \\
\text { facilities }\end{array}$} & \multirow{2}{*}{$\begin{array}{l}\text { Fortification } \\
\text { Cost }\end{array}$} & \multicolumn{2}{|c|}{ LP/NLP-BB } & \multicolumn{2}{|c|}{$\mathrm{OA}$} \\
\hline Size & $\beta$ & $\theta$ & & & & $\begin{array}{l}\text { Time } \\
\text { (Sec.) }\end{array}$ & $\begin{array}{l}\text { Objective } \\
\text { Value }\end{array}$ & $\begin{array}{l}\text { Time } \\
\text { (Sec.) }\end{array}$ & $\begin{array}{c}\text { Objective } \\
\text { Value }\end{array}$ \\
\hline $\begin{array}{c}49- \\
\text { node }\end{array}$ & 0.001 & 0.1 & 4 & 4 & 120 & 1.14 & 5569.56 & 4.44 & 5569.56 \\
\hline $\begin{array}{c}49- \\
\text { node }\end{array}$ & 0.002 & 0.1 & 5 & 5 & 150 & 0.75 & 8455.71 & 2.88 & 8455.71 \\
\hline $\begin{array}{c}49- \\
\text { node }\end{array}$ & 0.003 & 0.1 & 8 & 8 & 240 & 0.59 & 10689.97 & 3.99 & 10689.97 \\
\hline $\begin{array}{c}49- \\
\text { node }\end{array}$ & 0.004 & 0.1 & 8 & 8 & 240 & 0.61 & 12500.04 & 3.02 & 12500.04 \\
\hline $\begin{array}{c}49- \\
\text { node }\end{array}$ & 0.005 & 0.1 & 11 & 11 & 330 & 0.61 & 13917.04 & 3.08 & 13917.04 \\
\hline $\begin{array}{c}49- \\
\text { node }\end{array}$ & 0.002 & 0.2 & 5 & 5 & 150 & 0.75 & 8587.08 & 4.41 & 8587.08 \\
\hline $\begin{array}{c}49- \\
\text { node }\end{array}$ & 0.005 & 0.5 & 11 & 11 & 330 & 0.61 & 14492.96 & 5.06 & 14492.96 \\
\hline $\begin{array}{c}49- \\
\text { node }\end{array}$ & 0.005 & 1 & 11 & 11 & 330 & 0.69 & 14924.51 & 4.91 & 14924.51 \\
\hline $\begin{array}{c}49- \\
\text { node }\end{array}$ & 0.005 & 5 & 9 & 9 & 270 & 0.69 & 16677.22 & 34.39 & 16677.22 \\
\hline $\begin{array}{c}49- \\
\text { node }\end{array}$ & 0.005 & 10 & 8 & 8 & 240 & 0.84 & 17866.32 & 22.64 & 17866.32 \\
\hline $\begin{array}{c}49- \\
\text { node }\end{array}$ & 0.005 & 20 & 8 & 8 & 240 & 1.02 & 19506.61 & 59.33 & 19506.61 \\
\hline $\begin{array}{c}88- \\
\text { node }\end{array}$ & 0.001 & 0.1 & 9 & 9 & 270 & 4.28 & 13107.92 & 21.67 & 13107.92 \\
\hline $\begin{array}{c}88- \\
\text { node }\end{array}$ & 0.002 & 0.1 & 10 & 10 & 300 & 1.05 & 19843.23 & 16.545 & 19843.23 \\
\hline $\begin{array}{c}88- \\
\text { node }\end{array}$ & 0.003 & 0.1 & 15 & 12 & 360 & 2.97 & 25239.18 & 24.297 & 25239.18 \\
\hline $\begin{array}{c}88- \\
\text { node }\end{array}$ & 0.004 & 0.1 & 21 & 12 & 360 & 3.52 & 28919.25 & 55.453 & 28919.25 \\
\hline $\begin{array}{c}88- \\
\text { node }\end{array}$ & 0.005 & 0.1 & 23 & 12 & 360 & 2.94 & 31724.91 & 35.063 & 31724.91 \\
\hline $\begin{array}{c}88- \\
\text { node }\end{array}$ & 0.002 & 0.2 & 10 & 10 & 300 & 2.75 & 20231 & 16.468 & 20231 \\
\hline $\begin{array}{c}88- \\
\text { node }\end{array}$ & 0.005 & 0.5 & 23 & 12 & 360 & 2.92 & 33366.32 & 203.611 & 33366.32 \\
\hline $\begin{array}{c}88- \\
\text { node }\end{array}$ & 0.005 & 1 & 22 & 12 & 360 & 3.56 & 34578.01 & 165.437 & 34578.01 \\
\hline $\begin{array}{c}88- \\
\text { node }\end{array}$ & 0.005 & 5 & 20 & 12 & 360 & 3.63 & 38489.53 & $>600$ & 39198.52 \\
\hline $\begin{array}{c}88- \\
\text { node }\end{array}$ & 0.005 & 10 & 18 & 12 & 360 & 3.88 & 43249.78 & $>600$ & 44087.61 \\
\hline $\begin{array}{c}88- \\
\text { node }\end{array}$ & 0.005 & 20 & 15 & 12 & 360 & 4.72 & 46891.84 & $>600$ & 47780.48 \\
\hline
\end{tabular}


Table 5.11.a. LP/NLP-BB computational performance for 150-node problems, $q=0.05, R=3$

\begin{tabular}{|c|c|c|c|c|c|c|c|c|c|c|}
\hline \multicolumn{3}{|c|}{ Problem Instance } & \multirow{2}{*}{$\begin{array}{c}\text { \# } \\
\text { Opened } \\
\text { facilities }\end{array}$} & \multirow{2}{*}{$\begin{array}{l}\text { Time } \\
\text { (Sec.) }\end{array}$} & \multirow{2}{*}{$\begin{array}{c}\text { Optimality } \\
\text { Gap (\%) }\end{array}$} & \multirow{2}{*}{$\begin{array}{l}\text { Objective } \\
\text { Value }\end{array}$} & \multirow{2}{*}{$\begin{array}{c}\text { Fixed } \\
\text { Set-up } \\
\text { cost }(\%)\end{array}$} & \multirow{2}{*}{$\begin{array}{l}\text { Inventory } \\
\text { cost }(\%)\end{array}$} & \multirow{2}{*}{$\begin{array}{l}\text { Shipment } \\
\text { cost }(\%)\end{array}$} & \multirow{2}{*}{$\begin{array}{l}\text { Penalty } \\
\text { cost }(\%)\end{array}$} \\
\hline$B$ & $\beta$ & $\theta$ & & & & & & & & \\
\hline 0 & 0.0004 & 0.01 & 29 & 11.19 & 0.16 & 8668.45 & 33.45 & 10.29 & 33.91 & 22.34 \\
\hline 0 & 0.0005 & 0.01 & 31 & 9.24 & 0.08 & 9385.19 & 33.03 & 9.81 & 36.52 & 20.63 \\
\hline 0 & 0.0006 & 0.01 & 31 & 10.25 & 0.17 & 10068.15 & 30.79 & 9.16 & 40.81 & 19.23 \\
\hline 0 & 0.0008 & 0.01 & 41 & 3.52 & 0 & 11285.36 & 36.33 & 9.29 & 37.22 & 17.16 \\
\hline 0 & 0.001 & 0.01 & 50 & 7.27 & 0.03 & 12728.45 & 39.28 & 7.38 & 38.13 & 15.21 \\
\hline 0 & 0.001 & 0.02 & 51 & 2.97 & 0 & 12804.07 & 39.83 & 12.80 & 32.25 & 15.12 \\
\hline 0 & 0.001 & 0.1 & 50 & 10.34 & 0.42 & 14764.79 & 33.86 & 20.08 & 32.94 & 13.12 \\
\hline 0 & 0.001 & 0.5 & 49 & 19.03 & 0.68 & 19413.48 & 25.24 & 35.28 & 28.97 & 10.52 \\
\hline 0 & 0.001 & 1 & 30 & 99.36 & 0.91 & 22980.44 & 13.05 & 42.65 & 35.12 & 9.19 \\
\hline 0 & 0.002 & 0.04 & 70 & 3.24 & 0 & 17988.15 & 38.91 & 19.10 & 30.07 & 11.92 \\
\hline 30 & 0.0004 & 0.01 & 30 & 50.22 & 0 & 8531.61 & 35.16 & 10.74 & 33.60 & 20.49 \\
\hline 30 & 0.0005 & 0.01 & 34 & 63.61 & 0 & 9174.82 & 37.06 & 10.50 & 33.22 & 19.22 \\
\hline 30 & 0.0006 & 0.01 & 36 & 54.01 & 0 & 9804.04 & 36.72 & 10.14 & 35.15 & 17.99 \\
\hline 30 & 0.0008 & 0.01 & 41 & 42.45 & 0 & 10948.38 & 37.45 & 9.59 & 36.85 & 16.11 \\
\hline 30 & 0.001 & 0.01 & 48 & 37.41 & 0 & 11939.22 & 40.20 & 9.44 & 35.56 & 14.80 \\
\hline 30 & 0.001 & 0.02 & 48 & 39.66 & 0 & 12406.17 & 38.69 & 12.85 & 34.22 & 14.24 \\
\hline 30 & 0.001 & 0.1 & 48 & 49.21 & 0 & 14376.81 & 33.39 & 24.80 & 29.53 & 12.29 \\
\hline 30 & 0.001 & 0.5 & 48 & 88.00 & 0 & 18783.3 & 25.55 & 42.44 & 22.60 & 9.41 \\
\hline 30 & 0.001 & 1 & 30 & 126.37 & 0 & 21939.99 & 13.67 & 41.49 & 36.72 & 8.12 \\
\hline 30 & 0.002 & 0.04 & 70 & 33.66 & 0 & 16747.9 & 41.80 & 16.28 & 30.98 & 10.94 \\
\hline
\end{tabular}


Table 5.11.b. LP/NLP-BB computational performance for 150-node problems, $q=0.05, R=3$

\begin{tabular}{|c|c|c|c|c|c|c|c|c|c|c|}
\hline \multicolumn{3}{|c|}{ Problem Instance } & \multirow{2}{*}{\begin{tabular}{|c|}
$\#$ \\
Opened \\
facilities \\
\end{tabular}} & \multirow{2}{*}{$\begin{array}{l}\text { Time } \\
\text { (Sec.) }\end{array}$} & \multirow{2}{*}{$\begin{array}{c}\text { Optimality } \\
\text { Gap (\%) }\end{array}$} & \multirow{2}{*}{$\begin{array}{l}\text { Objective } \\
\text { Value }\end{array}$} & \multirow{2}{*}{$\begin{array}{c}\text { Fixed } \\
\text { Set-up } \\
\text { cost }(\%)\end{array}$} & \multirow{2}{*}{$\begin{array}{c}\text { Inventory } \\
\text { cost }(\%)\end{array}$} & \multirow{2}{*}{$\begin{array}{l}\text { Shipment } \\
\text { cost }(\%)\end{array}$} & \multirow{2}{*}{$\begin{array}{c}\text { Penalty } \\
\text { cost } \\
(\%) \\
\end{array}$} \\
\hline$B$ & $\beta$ & $\theta$ & & & & & & & & \\
\hline 60 & 0.0004 & 0.01 & 29 & 62.86 & 0 & 8358.27 & 34.69 & 10.56 & 33.90 & 20.85 \\
\hline 60 & 0.0005 & 0.01 & 33 & 64.44 & 0 & 8977.28 & 36.76 & 10.57 & 34.65 & 18.03 \\
\hline 60 & 0.0006 & 0.01 & 36 & 55.70 & 0 & 9629.16 & 37.39 & 10.34 & 35.41 & 16.87 \\
\hline 60 & 0.0008 & 0.01 & 41 & 69.89 & 0 & 10643.46 & 38.52 & 8.30 & 39.33 & 13.84 \\
\hline 60 & 0.001 & 0.01 & 48 & 37.91 & 0 & 11855.03 & 40.49 & 9.51 & 35.41 & 14.59 \\
\hline 60 & 0.001 & 0.02 & 48 & 40.00 & 0 & 12321.86 & 38.96 & 12.94 & 34.07 & 14.04 \\
\hline 60 & 0.001 & 0.1 & 48 & 49.59 & 0 & 14291.97 & 33.59 & 24.94 & 29.37 & 12.10 \\
\hline 60 & 0.001 & 0.5 & 48 & 88.72 & 0 & 18697.28 & 25.67 & 42.62 & 22.45 & 9.25 \\
\hline 60 & 0.001 & 1 & 30 & 129.82 & 0 & 21481.26 & 14.90 & 43.25 & 35.10 & 6.76 \\
\hline 60 & 0.002 & 0.04 & 69 & 33.67 & 0 & 16531.58 & 41.74 & 16.30 & 31.63 & 10.33 \\
\hline 120 & 0.0004 & 0.01 & 28 & 88.72 & 0 & 7411.05 & 37.78 & 11.64 & 40.64 & 9.94 \\
\hline 120 & 0.0005 & 0.01 & 32 & 65.28 & 0 & 8355.03 & 38.30 & 11.20 & 37.62 & 12.88 \\
\hline 120 & 0.0006 & 0.01 & 35 & 57.77 & 0 & 8968.62 & 39.02 & 10.84 & 38.40 & 11.73 \\
\hline 120 & 0.0008 & 0.01 & 40 & 146.77 & 0.49 & 10805.81 & 37.02 & 6.94 & 47.27 & 8.77 \\
\hline 120 & 0.001 & 0.01 & 48 & 129.36 & 0.05 & 11915.5 & 40.28 & 5.85 & 46.87 & 7.00 \\
\hline 120 & 0.001 & 0.02 & 48 & 102.74 & 0 & 12248.99 & 39.19 & 9.35 & 43.29 & 8.17 \\
\hline 120 & 0.001 & 0.1 & 48 & 125.74 & 0 & 14133.82 & 33.96 & 18.33 & 41.28 & 6.42 \\
\hline 120 & 0.001 & 0.5 & 49 & 204.66 & 0.57 & 18767.09 & 26.11 & 29.85 & 38.99 & 5.04 \\
\hline 120 & 0.001 & 1 & 30 & 109.30 & 0 & 20502.42 & 14.63 & 43.71 & 35.95 & 5.71 \\
\hline 120 & 0.002 & 0.04 & 69 & 37.70 & 0 & 16493.49 & 41.83 & 18.40 & 32.14 & 7.64 \\
\hline
\end{tabular}


Table 5.11.c. LP/NLP-BB computational performance for 150-node problems, $q=0.05, R=3$

\begin{tabular}{|c|c|c|c|c|c|c|c|c|c|c|}
\hline \multicolumn{3}{|c|}{ Problem Instance } & \multirow{2}{*}{$\begin{array}{c}\# \\
\text { Opened } \\
\text { facilities } \\
\end{array}$} & \multirow{2}{*}{$\begin{array}{l}\text { Time } \\
\text { (Sec.) }\end{array}$} & \multirow{2}{*}{$\begin{array}{c}\text { Optimality } \\
\text { Gap (\%) }\end{array}$} & \multirow{2}{*}{$\begin{array}{c}\text { Objective } \\
\text { Value }\end{array}$} & \multirow{2}{*}{$\begin{array}{c}\text { Fixed } \\
\text { Set-up } \\
\text { cost }(\%) \\
\end{array}$} & \multirow{2}{*}{$\begin{array}{c}\text { Inventory } \\
\text { cost }(\%)\end{array}$} & \multirow{2}{*}{$\begin{array}{c}\text { Shipment } \\
\text { cost }(\%)\end{array}$} & \multirow{2}{*}{$\begin{array}{l}\text { Penalty } \\
\text { cost }(\%)\end{array}$} \\
\hline$B$ & $\beta$ & $\theta$ & & & & & & & & \\
\hline 180 & 0.0004 & 0.01 & 28 & 63.41 & 0 & 7042.23 & 39.76 & 12.47 & 39.91 & 7.86 \\
\hline 180 & 0.0005 & 0.01 & 32 & 77.81 & 0 & 8255.62 & 38.76 & 10.92 & 40.06 & 10.26 \\
\hline 180 & 0.0006 & 0.01 & 35 & 44.61 & 0 & 7871.86 & 44.46 & 12.34 & 43.20 & 0 \\
\hline 180 & 0.0008 & 0.01 & 40 & 37.84 & 0 & 9000.26 & 44.44 & 11.46 & 44.10 & 0 \\
\hline 180 & 0.001 & 0.01 & 47 & 35.80 & 0 & 9986.17 & 47.07 & 11.06 & 41.87 & 0 \\
\hline 180 & 0.001 & 0.02 & 47 & 37.33 & 0 & 10443.83 & 45.00 & 14.96 & 40.04 & 0 \\
\hline 180 & 0.001 & 0.1 & 46 & 60.59 & 0 & 13576.43 & 33.68 & 24.35 & 34.84 & 6.93 \\
\hline 180 & 0.001 & 0.5 & 47 & 287.47 & 0.84 & 18734.25 & 25.09 & 30.71 & 41.70 & 2.49 \\
\hline 180 & 0.001 & 1 & 30 & 254.29 & 0 & 20390.34 & 14.71 & 47.95 & 33.02 & 4.32 \\
\hline 180 & 0.002 & 0.04 & 69 & 60.72 & 0 & 16351.31 & 42.20 & 18.25 & 35.22 & 4.33 \\
\hline 240 & 0.0004 & 0.01 & 28 & 54.41 & 0 & 6446.59 & 43.43 & 13.61 & 42.96 & 0 \\
\hline 240 & 0.0005 & 0.01 & 30 & 57.27 & 0 & 7140.71 & 42.01 & 12.60 & 45.39 & 0 \\
\hline 240 & 0.0006 & 0.01 & 35 & 46.74 & 0 & 7809.32 & 44.82 & 12.43 & 42.76 & 0 \\
\hline 240 & 0.0008 & 0.01 & 39 & 34.34 & 0 & 8909.6 & 43.77 & 11.45 & 44.78 & 0 \\
\hline 240 & 0.001 & 0.01 & 47 & 28.00 & 0 & 9902.13 & 47.46 & 11.15 & 41.39 & 0 \\
\hline 240 & 0.001 & 0.02 & 47 & 30.22 & 0 & 10359.3 & 45.37 & 15.07 & 39.56 & 0 \\
\hline 240 & 0.001 & 0.1 & 46 & 44.35 & 0 & 12288.64 & 37.43 & 28.40 & 34.17 & 0 \\
\hline 240 & 0.001 & 0.5 & 46 & 99.42 & 0 & 16602.77 & 27.10 & 47.01 & 25.90 & 0 \\
\hline 240 & 0.001 & 1 & 30 & 141.86 & 0 & 20125.76 & 14.91 & 45.74 & 35.88 & 3.47 \\
\hline 240 & 0.002 & 0.04 & 68 & 28.66 & 0 & 15632.13 & 43.50 & 18.09 & 38.40 & 0 \\
\hline
\end{tabular}


Table 5.11.d. LP/NLP-BB computational performance for 150-node problems, $q=0.05, R=3$

\begin{tabular}{|c|c|c|c|c|c|c|c|c|c|c|}
\hline \multicolumn{3}{|c|}{ Problem Instance } & \multirow{2}{*}{$\begin{array}{c}\# \\
\text { Opened } \\
\text { facilities }\end{array}$} & \multirow{2}{*}{$\begin{array}{l}\text { Time } \\
\text { (Sec.) }\end{array}$} & \multirow{2}{*}{$\begin{array}{c}\text { Optimality } \\
\text { Gap }(\%)\end{array}$} & \multirow{2}{*}{$\begin{array}{c}\text { Objective } \\
\text { Value }\end{array}$} & \multirow{2}{*}{$\begin{array}{c}\text { Fixed } \\
\text { Set-up } \\
\text { cost }(\%)\end{array}$} & \multirow{2}{*}{$\begin{array}{c}\text { Inventory } \\
\text { cost }(\%)\end{array}$} & \multirow{2}{*}{$\begin{array}{l}\text { Shipment } \\
\text { cost }(\%)\end{array}$} & \multirow{2}{*}{$\begin{array}{l}\text { Penalty } \\
\text { cost }(\%)\end{array}$} \\
\hline$B$ & $\beta$ & $\theta$ & & & & & & & & \\
\hline 300 & 0.0004 & 0.01 & 28 & 54.55 & 0 & 6405.77 & 43.71 & 13.69 & 42.60 & 0 \\
\hline 300 & 0.0005 & 0.01 & 30 & 54.09 & 0 & 7089.79 & 42.31 & 12.68 & 45.00 & 0 \\
\hline 300 & 0.0006 & 0.01 & 35 & 48.61 & 0 & 7750.67 & 45.16 & 12.52 & 42.32 & 0 \\
\hline 300 & 0.0008 & 0.01 & 39 & 22.58 & 0 & 8841.29 & 44.11 & 11.53 & 44.36 & 0 \\
\hline 300 & 0.001 & 0.01 & 47 & 19.75 & 0 & 9840.36 & 47.76 & 11.21 & 41.03 & 0 \\
\hline 300 & 0.001 & 0.02 & 47 & 22.69 & 0 & 10297.36 & 45.64 & 15.15 & 39.20 & 0 \\
\hline 300 & 0.001 & 0.1 & 46 & 38.36 & 0 & 12225.98 & 37.62 & 28.54 & 33.84 & 0 \\
\hline 300 & 0.001 & 0.5 & 46 & 100.64 & 0 & 16538.51 & 27.81 & 47.17 & 25.02 & 0 \\
\hline 300 & 0.001 & 1 & 30 & 136.84 & 0 & 19943.17 & 15.04 & 46.14 & 35.65 & 3.16 \\
\hline 300 & 0.002 & 0.04 & 68 & 28.97 & 0 & 14709.44 & 46.23 & 18.00 & 35.77 & 0 \\
\hline 360 & 0.0004 & 0.01 & 28 & 59.51 & 0 & 6369.35 & 43.96 & 13.78 & 42.26 & 0 \\
\hline 360 & 0.0005 & 0.01 & 30 & 45.16 & 0 & 7045.63 & 42.58 & 12.77 & 44.65 & 0 \\
\hline 360 & 0.0006 & 0.01 & 34 & 41.39 & 0 & 7695.42 & 44.18 & 12.45 & 43.37 & 0 \\
\hline 360 & 0.0008 & 0.01 & 39 & 23.83 & 0 & 8793.27 & 44.35 & 11.58 & 44.06 & 0 \\
\hline 360 & 0.001 & 0.01 & 46 & 22.66 & 0 & 9770.18 & 47.08 & 11.13 & 41.79 & 0 \\
\hline 360 & 0.001 & 0.02 & 46 & 26.02 & 0 & 10220.65 & 45.01 & 15.05 & 39.95 & 0 \\
\hline 360 & 0.001 & 0.1 & 46 & 41.25 & 0 & 12121.7 & 37.95 & 28.37 & 33.68 & 0 \\
\hline 360 & 0.001 & 0.5 & 46 & 93.91 & 0 & 16372.58 & 28.10 & 46.97 & 24.94 & 0 \\
\hline 360 & 0.001 & 1 & 30 & 129.28 & 0 & 19076.17 & 15.73 & 47.03 & 36.41 & 0.84 \\
\hline 360 & 0.002 & 0.04 & 67 & 22.45 & 0 & 14413.59 & 46.48 & 18.23 & 35.28 & 0 \\
\hline
\end{tabular}


Table 5.12.a. LP/NLP-BB computational performance for 150-node problems, $q=0.05, R=2$

\begin{tabular}{|c|c|c|c|c|c|c|c|c|c|c|}
\hline \multicolumn{3}{|c|}{ Problem Instance } & \multirow{2}{*}{$\begin{array}{c}\text { \# } \\
\text { Opened } \\
\text { facilities }\end{array}$} & \multirow{2}{*}{$\begin{array}{l}\text { Time } \\
(\text { Sec. })\end{array}$} & \multirow{2}{*}{$\begin{array}{c}\text { Optimality } \\
\text { Gap (\%) }\end{array}$} & \multirow{2}{*}{$\begin{array}{l}\text { Objective } \\
\text { Value }\end{array}$} & \multirow{2}{*}{$\begin{array}{c}\text { Fixed } \\
\text { Set-up } \\
\text { cost }(\%)\end{array}$} & \multirow{2}{*}{$\begin{array}{c}\text { Inventory } \\
\text { cost }(\%)\end{array}$} & \multirow{2}{*}{$\begin{array}{c}\text { Shipment } \\
\text { cost }(\%)\end{array}$} & \multirow{2}{*}{$\begin{array}{l}\text { Penalty } \\
\text { cost }(\%)\end{array}$} \\
\hline$B$ & $\beta$ & $\theta$ & & & & & & & & \\
\hline 0 & 0.0004 & 0.01 & 28 & 7.79 & 0.59 & 22677.84 & 12.35 & 4.01 & 15.32 & 68.32 \\
\hline 0 & 0.0005 & 0.01 & 30 & 5.59 & 0.06 & 23517.17 & 12.76 & 4.08 & 17.28 & 65.88 \\
\hline 0 & 0.0006 & 0.01 & 30 & 4.48 & 0.08 & 24269.53 & 12.36 & 4.03 & 19.77 & 63.84 \\
\hline 0 & 0.0008 & 0.01 & 40 & 2.12 & 0 & 25611.82 & 15.62 & 4.42 & 19.46 & 60.49 \\
\hline 0 & 0.001 & 0.01 & 50 & 3.12 & 0 & 26915.07 & 18.58 & 4.14 & 19.72 & 57.56 \\
\hline 0 & 0.001 & 0.02 & 50 & 1.79 & 0 & 27177.07 & 18.40 & 6.36 & 18.24 & 57.01 \\
\hline 0 & 0.001 & 0.1 & 49 & 6.01 & 0.23 & 29218.63 & 16.77 & 11.17 & 19.04 & 53.02 \\
\hline 0 & 0.001 & 0.5 & 48 & 10.75 & 0.46 & 33015.17 & 14.54 & 20.59 & 17.94 & 46.93 \\
\hline 0 & 0.001 & 1 & 29 & 27.47 & 0.87 & 37590.20 & 7.71 & 26.43 & 23.87 & 42.00 \\
\hline 0 & 0.002 & 0.04 & 69 & 2.10 & 0 & 33796.23 & 20.42 & 14.43 & 19.31 & 45.84 \\
\hline 30 & 0.0004 & 0.01 & 29 & 14.24 & 0 & 21515.8 & 13.48 & 4.24 & 16.04 & 66.24 \\
\hline 30 & 0.0005 & 0.01 & 33 & 39.61 & 0 & 21991.42 & 15.01 & 4.50 & 16.35 & 64.15 \\
\hline 30 & 0.0006 & 0.01 & 34 & 37.20 & 0 & 22689.42 & 14.98 & 4.46 & 18.38 & 62.18 \\
\hline 30 & 0.0008 & 0.01 & 40 & 38.61 & 0 & 24583.22 & 16.27 & 3.96 & 22.08 & 57.69 \\
\hline 30 & 0.001 & 0.01 & 48 & 32.98 & 0 & 26343.61 & 18.22 & 4.19 & 22.43 & 56.68 \\
\hline 30 & 0.001 & 0.02 & 47 & 33.03 & 0 & 26824.92 & 17.52 & 5.82 & 20.99 & 55.67 \\
\hline 30 & 0.001 & 0.1 & 47 & 35.42 & 0 & 28909.63 & 16.26 & 12.07 & 21.06 & 51.65 \\
\hline 30 & 0.001 & 0.5 & 47 & 40.90 & 0.64 & 29811.32 & 15.77 & 21.93 & 29.01 & 38.33 \\
\hline 30 & 0.001 & 1 & 29 & 25.23 & 0 & 37137.21 & 7.81 & 30.92 & 15.60 & 40.55 \\
\hline 30 & 0.002 & 0.04 & 69 & 31.75 & 0 & 33538.09 & 20.57 & 14.35 & 20.56 & 44.52 \\
\hline
\end{tabular}


Table 5.12.b. LP/NLP-BB computational performance for 150-node problems, $q=0.05, R=2$

\begin{tabular}{|c|c|c|c|c|c|c|c|c|c|c|}
\hline \multicolumn{3}{|c|}{ Problem Instance } & \multirow{2}{*}{$\begin{array}{c}\# \\
\text { Opened } \\
\text { facilities }\end{array}$} & \multirow{2}{*}{$\begin{array}{l}\text { Time } \\
\text { (Sec.) }\end{array}$} & \multirow{2}{*}{$\begin{array}{c}\text { Optimality } \\
\text { Gap (\%) }\end{array}$} & \multirow{2}{*}{$\begin{array}{c}\text { Objective } \\
\text { Value }\end{array}$} & \multirow{2}{*}{$\begin{array}{c}\text { Fixed } \\
\text { Set-up } \\
\text { cost }(\%)\end{array}$} & \multirow{2}{*}{$\begin{array}{l}\text { Inventory } \\
\text { cost }(\%)\end{array}$} & \multirow{2}{*}{$\begin{array}{l}\text { Shipment } \\
\text { cost }(\%)\end{array}$} & \multirow{2}{*}{$\begin{array}{l}\text { Penalty } \\
\text { cost }(\%)\end{array}$} \\
\hline$B$ & $\beta$ & $\theta$ & & & & & & & & \\
\hline 60 & 0.0004 & 0.01 & 28 & 40.64 & 0 & 19863.37 & 14.10 & 4.57 & 16.65 & 64.68 \\
\hline 60 & 0.0005 & 0.01 & 33 & 39.69 & 0 & 20743.61 & 15.91 & 4.68 & 16.99 & 62.42 \\
\hline 60 & 0.0006 & 0.01 & 33 & 36.33 & 0 & 20551.94 & 16.06 & 4.73 & 24.33 & 54.88 \\
\hline 60 & 0.0008 & 0.01 & 38 & 44.11 & 0 & 22332.55 & 17.02 & 4.37 & 27.79 & 50.81 \\
\hline 60 & 0.001 & 0.01 & 48 & 32.98 & 0 & 25391.33 & 18.90 & 4.30 & 21.57 & 55.23 \\
\hline 60 & 0.001 & 0.02 & 47 & 33.01 & 0 & 25867.68 & 18.17 & 5.97 & 21.65 & 54.21 \\
\hline 60 & 0.001 & 0.1 & 46 & 35.56 & 0 & 26189.83 & 17.56 & 11.84 & 27.85 & 42.74 \\
\hline 60 & 0.001 & 0.5 & 46 & 41.37 & 0.93 & 28112.07 & 16.36 & 23.31 & 23.10 & 37.23 \\
\hline 60 & 0.001 & 1 & 29 & 22.36 & 0 & 36717.87 & 7.90 & 29.70 & 28.57 & 33.83 \\
\hline 60 & 0.002 & 0.04 & 69 & 31.77 & 0 & 33326.61 & 20.70 & 14.05 & 30.18 & 35.07 \\
\hline 120 & 0.0004 & 0.01 & 28 & 40.01 & 0 & 19190.76 & 14.59 & 5.24 & 21.53 & 58.64 \\
\hline 120 & 0.0005 & 0.01 & 32 & 45.52 & 0 & 19181.84 & 16.68 & 5.12 & 29.15 & 49.05 \\
\hline 120 & 0.0006 & 0.01 & 32 & 44.66 & 0 & 19972.2 & 16.02 & 5.10 & 29.26 & 49.62 \\
\hline 120 & 0.0008 & 0.01 & 38 & 34.26 & 0 & 20812.92 & 18.26 & 4.40 & 32.33 & 45.01 \\
\hline 120 & 0.001 & 0.01 & 47 & 33.12 & 0 & 23421.49 & 20.07 & 4.26 & 33.69 & 41.98 \\
\hline 120 & 0.001 & 0.02 & 47 & 33.23 & 0 & 23799.56 & 19.75 & 5.91 & 33.09 & 41.25 \\
\hline 120 & 0.001 & 0.1 & 45 & 35.83 & 0 & 25324.21 & 17.77 & 11.44 & 36.92 & 33.88 \\
\hline 120 & 0.001 & 0.5 & 45 & 43.91 & 0 & 27075.4 & 16.62 & 23.17 & 24.90 & 35.31 \\
\hline 120 & 0.001 & 1 & 28 & 52.87 & 0 & 33861.21 & 8.27 & 30.27 & 30.35 & 31.11 \\
\hline 120 & 0.002 & 0.04 & 69 & 31.79 & 0 & 31349.42 & 22.01 & 15.20 & 36.11 & 28.68 \\
\hline
\end{tabular}


Table 5.12.c. LP/NLP-BB computational performance for 150-node problems, $q=0.05, R=2$

\begin{tabular}{|c|c|c|c|c|c|c|c|c|c|c|}
\hline \multicolumn{3}{|c|}{ Problem Instance } & \multirow{2}{*}{$\begin{array}{c}\# \\
\text { Opened } \\
\text { facilities }\end{array}$} & \multirow{2}{*}{$\begin{array}{l}\text { Time } \\
\text { (Sec.) }\end{array}$} & \multirow{2}{*}{$\begin{array}{c}\text { Optimality } \\
\text { Gap }(\%)\end{array}$} & \multirow{2}{*}{$\begin{array}{c}\text { Objective } \\
\text { Value }\end{array}$} & \multirow{2}{*}{$\begin{array}{c}\text { Fixed } \\
\text { Set-up } \\
\text { cost }(\%)\end{array}$} & \multirow{2}{*}{$\begin{array}{l}\text { Inventory } \\
\text { cost }(\%)\end{array}$} & \multirow{2}{*}{$\begin{array}{c}\text { Shipment } \\
\text { cost }(\%)\end{array}$} & \multirow{2}{*}{$\begin{array}{l}\text { Penalty } \\
\text { cost }(\%)\end{array}$} \\
\hline$B$ & $\beta$ & $\theta$ & & & & & & & & \\
\hline 180 & 0.0004 & 0.01 & 28 & 32.16 & 0 & 18910.15 & 14.81 & 5.92 & 27.74 & 51.52 \\
\hline 180 & 0.0005 & 0.01 & 32 & 35.14 & 0 & 18916.27 & 16.92 & 5.61 & 28.09 & 49.39 \\
\hline 180 & 0.0006 & 0.01 & 32 & 39.33 & 0 & 19520.93 & 16.39 & 5.32 & 22.79 & 55.51 \\
\hline 180 & 0.0008 & 0.01 & 38 & 34.62 & 0 & 20401.33 & 18.63 & 4.55 & 39.22 & 37.61 \\
\hline 180 & 0.001 & 0.01 & 47 & 33.19 & 0 & 21639.91 & 21.72 & 4.45 & 40.90 & 32.93 \\
\hline 180 & 0.001 & 0.02 & 47 & 34.16 & 0 & 21710.25 & 21.65 & 5.47 & 53.94 & 18.94 \\
\hline 180 & 0.001 & 0.1 & 45 & 36.72 & 0 & 23353.09 & 19.27 & 12.18 & 47.10 & 21.45 \\
\hline 180 & 0.001 & 0.5 & 45 & 46.75 & 0 & 26154.61 & 17.21 & 25.16 & 26.41 & 31.22 \\
\hline 180 & 0.001 & 1 & 28 & 60.09 & 0 & 31392.05 & 8.92 & 30.21 & 35.03 & 25.84 \\
\hline 180 & 0.002 & 0.04 & 69 & 31.88 & 0 & 30177.72 & 22.86 & 15.63 & 38.52 & 23 \\
\hline 240 & 0.0004 & 0.01 & 27 & 41.28 & 0 & 6723.88 & 40.16 & 13 & 46.84 & 0 \\
\hline 240 & 0.0005 & 0.01 & 31 & 40.33 & 0 & 7465.12 & 41.53 & 12.06 & 46.41 & 0 \\
\hline 240 & 0.0006 & 0.01 & 30 & 38.05 & 0 & 8163.66 & 36.75 & 11.95 & 51.30 & 0 \\
\hline 240 & 0.0008 & 0.01 & 38 & 34.78 & 0 & 16980.9 & 22.38 & 5.26 & 34.04 & 38.32 \\
\hline 240 & 0.001 & 0.01 & 46 & 33.31 & 0 & 19059.11 & 24.14 & 4.86 & 35.45 & 35.56 \\
\hline 240 & 0.001 & 0.02 & 46 & 34.39 & 0 & 19369.1 & 23.75 & 6.76 & 34.89 & 34.6 \\
\hline 240 & 0.001 & 0.1 & 44 & 36.98 & 0 & 20943.22 & 21.01 & 14.27 & 33.29 & 31.43 \\
\hline 240 & 0.001 & 0.5 & 44 & 49.47 & 0 & 25218.83 & 17.45 & 25.95 & 27.90 & 28.7 \\
\hline 240 & 0.001 & 1 & 27 & 57.79 & 0 & 28482.46 & 9.48 & 33.13 & 31.90 & 25.5 \\
\hline 240 & 0.002 & 0.04 & 69 & 31.91 & 0 & 28313.07 & 24.37 & 16.85 & 34.85 & 23.93 \\
\hline
\end{tabular}


Table 5.12.d. LP/NLP-BB computational performance for 150-node problems, $q=0.05, R=2$

\begin{tabular}{|c|c|c|c|c|c|c|c|c|c|c|}
\hline \multicolumn{3}{|c|}{ Problem Instance } & \multirow{2}{*}{$\begin{array}{c}\# \\
\text { Opened } \\
\text { facilities }\end{array}$} & \multirow{2}{*}{$\begin{array}{l}\text { Time } \\
\text { (Sec.) }\end{array}$} & \multirow{2}{*}{$\begin{array}{c}\text { Optimality } \\
\text { Gap (\%) }\end{array}$} & \multirow{2}{*}{$\begin{array}{l}\text { Objective } \\
\text { Value }\end{array}$} & \multirow{2}{*}{$\begin{array}{c}\text { Fixed } \\
\text { Set-up } \\
\text { cost }(\%)\end{array}$} & \multirow{2}{*}{$\begin{array}{l}\text { Inventory } \\
\text { cost }(\%)\end{array}$} & \multirow{2}{*}{$\begin{array}{l}\text { Shipment } \\
\text { cost }(\%)\end{array}$} & \multirow{2}{*}{$\begin{array}{l}\text { Penalty } \\
\text { cost }(\%)\end{array}$} \\
\hline$B$ & $\beta$ & $\theta$ & & & & & & & & \\
\hline 300 & 0.0004 & 0.01 & 27 & 46.31 & 0.57 & 6603.64 & 40.89 & 13.23 & 45.88 & 0 \\
\hline 300 & 0.0005 & 0.01 & 31 & 40.05 & 0 & 7327.5 & 42.31 & 12.28 & 45.41 & 0 \\
\hline 300 & 0.0006 & 0.01 & 30 & 13.3 & 0 & 8031.62 & 37.35 & 11.94 & 50.71 & 0 \\
\hline 300 & 0.0008 & 0.01 & 38 & 34.92 & 0 & 9217.37 & 41.23 & 11.09 & 47.68 & 0 \\
\hline 300 & 0.001 & 0.01 & 46 & 35.34 & 0 & 10880.84 & 42.28 & 10.42 & 47.30 & 0 \\
\hline 300 & 0.001 & 0.02 & 46 & 35.22 & 0 & 10727.36 & 42.88 & 14.22 & 42.90 & 0 \\
\hline 300 & 0.001 & 0.1 & 44 & 38.48 & 0 & 12612.8 & 34.89 & 27.04 & 38.07 & 0 \\
\hline 300 & 0.001 & 0.5 & 44 & 54.05 & 0 & 16828.78 & 26.15 & 45.32 & 28.53 & 0 \\
\hline 300 & 0.001 & 1 & 27 & 61.87 & 0 & 19987.9 & 13.51 & 53.96 & 32.52 & 0 \\
\hline 300 & 0.002 & 0.04 & 69 & 31.98 & 0 & 26411.44 & 26.13 & 16.36 & 38.17 & 19.33 \\
\hline 360 & 0.0004 & 0.01 & 27 & 35.50 & 0 & 6516.53 & 41.43 & 13.42 & 45.15 & 0 \\
\hline 360 & 0.0005 & 0.01 & 31 & 29.26 & 0 & 7233.84 & 42.85 & 12.45 & 44.70 & 0 \\
\hline 360 & 0.0006 & 0.01 & 30 & 36.13 & 0 & 7914.28 & 37.91 & 11.96 & 50.13 & 0 \\
\hline 360 & 0.0008 & 0.01 & 38 & 34.09 & 0 & 9107.71 & 41.72 & 11.21 & 47.07 & 0 \\
\hline 360 & 0.001 & 0.01 & 46 & 31.17 & 0 & 9536.17 & 48.24 & 5.89 & 22.21 & 0 \\
\hline 360 & 0.001 & 0.02 & 46 & 33.01 & 0 & 10703.06 & 42.98 & 13.87 & 43.16 & 0 \\
\hline 360 & 0.001 & 0.1 & 44 & 37.14 & 0 & 12569.66 & 35.00 & 26.53 & 38.46 & 0 \\
\hline 360 & 0.001 & 0.5 & 44 & 43.51 & 0.39 & 16638.98 & 26.44 & 44.59 & 28.96 & 0 \\
\hline 360 & 0.001 & 1 & 27 & 31.55 & 0 & 19756.39 & 13.67 & 53.38 & 32.94 & 0 \\
\hline 360 & 0.002 & 0.04 & 69 & 41.95 & 0 & 20032.2 & 34.44 & 16.08 & 46.39 & 3.09 \\
\hline
\end{tabular}


Table 5.13.a. LP/NLP-BB computational performance for 150-node problems, $q=0.05, R=4$

\begin{tabular}{|c|c|c|c|c|c|c|c|c|c|c|}
\hline \multicolumn{3}{|c|}{ Problem Instance } & \multirow{2}{*}{$\begin{array}{c}\# \\
\text { Opened } \\
\text { facilities } \\
\end{array}$} & \multirow{2}{*}{$\begin{array}{l}\text { Time } \\
\text { (Sec.) }\end{array}$} & \multirow{2}{*}{$\begin{array}{c}\text { Optimality } \\
\text { Gap }(\%)\end{array}$} & \multirow{2}{*}{$\begin{array}{c}\text { Objective } \\
\text { Value }\end{array}$} & \multirow{2}{*}{$\begin{array}{c}\text { Fixed } \\
\text { Set-up } \\
\text { cost }(\%)\end{array}$} & \multirow{2}{*}{$\begin{array}{l}\text { Inventory } \\
\text { cost }(\%)\end{array}$} & \multirow{2}{*}{$\begin{array}{l}\text { Shipment } \\
\text { cost }(\%)\end{array}$} & \multirow{2}{*}{$\begin{array}{l}\text { Penalty } \\
\text { cost }(\%)\end{array}$} \\
\hline$B$ & $\beta$ & $\theta$ & & & & & & & & \\
\hline 0 & 0.0004 & 0.01 & 29 & 17.89 & 0.16 & 6831.05 & 42.45 & 13.06 & 43.07 & 1.42 \\
\hline 0 & 0.0005 & 0.01 & 32 & 10.67 & 0 & 7701.24 & 41.55 & 12.19 & 45.00 & 1.26 \\
\hline 0 & 0.0006 & 0.01 & 32 & 10.12 & 0.05 & 8218.7 & 38.94 & 11.39 & 48.50 & 1.18 \\
\hline 0 & 0.0008 & 0.01 & 41 & 9.68 & 0.11 & 9553.35 & 42.92 & 10.87 & 45.20 & 1.01 \\
\hline 0 & 0.001 & 0.01 & 48 & 3.76 & 0 & 10226.6 & 46.94 & 11.03 & 42.03 & 0.00 \\
\hline 0 & 0.001 & 0.02 & 48 & 4.66 & 0 & 10693.83 & 44.89 & 14.92 & 40.20 & 0.00 \\
\hline 0 & 0.001 & 0.1 & 48 & 7.47 & 0 & 12665.62 & 37.90 & 28.16 & 33.94 & 0.00 \\
\hline 0 & 0.001 & 0.5 & 49 & 37.92 & 0.68 & 17217.53 & 28.46 & 36.59 & 34.38 & 0.56 \\
\hline 0 & 0.001 & 1 & 30 & 129.06 & 0.94 & 19813.48 & 15.14 & 44.70 & 39.67 & 0.49 \\
\hline 0 & 0.002 & 0.04 & 70 & 2.73 & 0 & 14994.85 & 46.68 & 18.20 & 35.12 & 0.00 \\
\hline 30 & 0.0004 & 0.01 & 29 & 67.92 & 0 & 6836.26 & 42.42 & 13.09 & 43.32 & 1.17 \\
\hline 30 & 0.0005 & 0.01 & 34 & 76.56 & 0 & 7497.43 & 45.35 & 12.85 & 40.69 & 1.11 \\
\hline 30 & 0.0006 & 0.01 & 36 & 69.18 & 0 & 8126.97 & 44.30 & 12.23 & 42.45 & 1.02 \\
\hline 30 & 0.0008 & 0.01 & 41 & 52.79 & 0 & 9276.39 & 44.20 & 11.32 & 43.54 & 0.94 \\
\hline 30 & 0.001 & 0.01 & 48 & 45.89 & 0 & 10163.32 & 47.23 & 10.98 & 40.94 & 0.84 \\
\hline 30 & 0.001 & 0.02 & 48 & 49.34 & 0 & 10730.31 & 44.73 & 14.86 & 39.60 & 0.81 \\
\hline 30 & 0.001 & 0.1 & 48 & 62.57 & 0 & 12701.06 & 37.79 & 28.07 & 33.46 & 0.68 \\
\hline 30 & 0.001 & 0.5 & 48 & 113.67 & 0 & 17107.82 & 28.06 & 46.6 & 24.84 & 0.51 \\
\hline 30 & 0.001 & 1 & 30 & 217.09 & 0 & 19609.88 & 15.30 & 55.24 & 29.04 & 0.42 \\
\hline 30 & 0.002 & 0.04 & 70 & 36.90 & 0 & 15012.61 & 46.63 & 18.17 & 34.60 & 0.61 \\
\hline
\end{tabular}


Table 5.13.b. LP/NLP-BB computational performance for 150-node problems, $q=0.05, R=4$

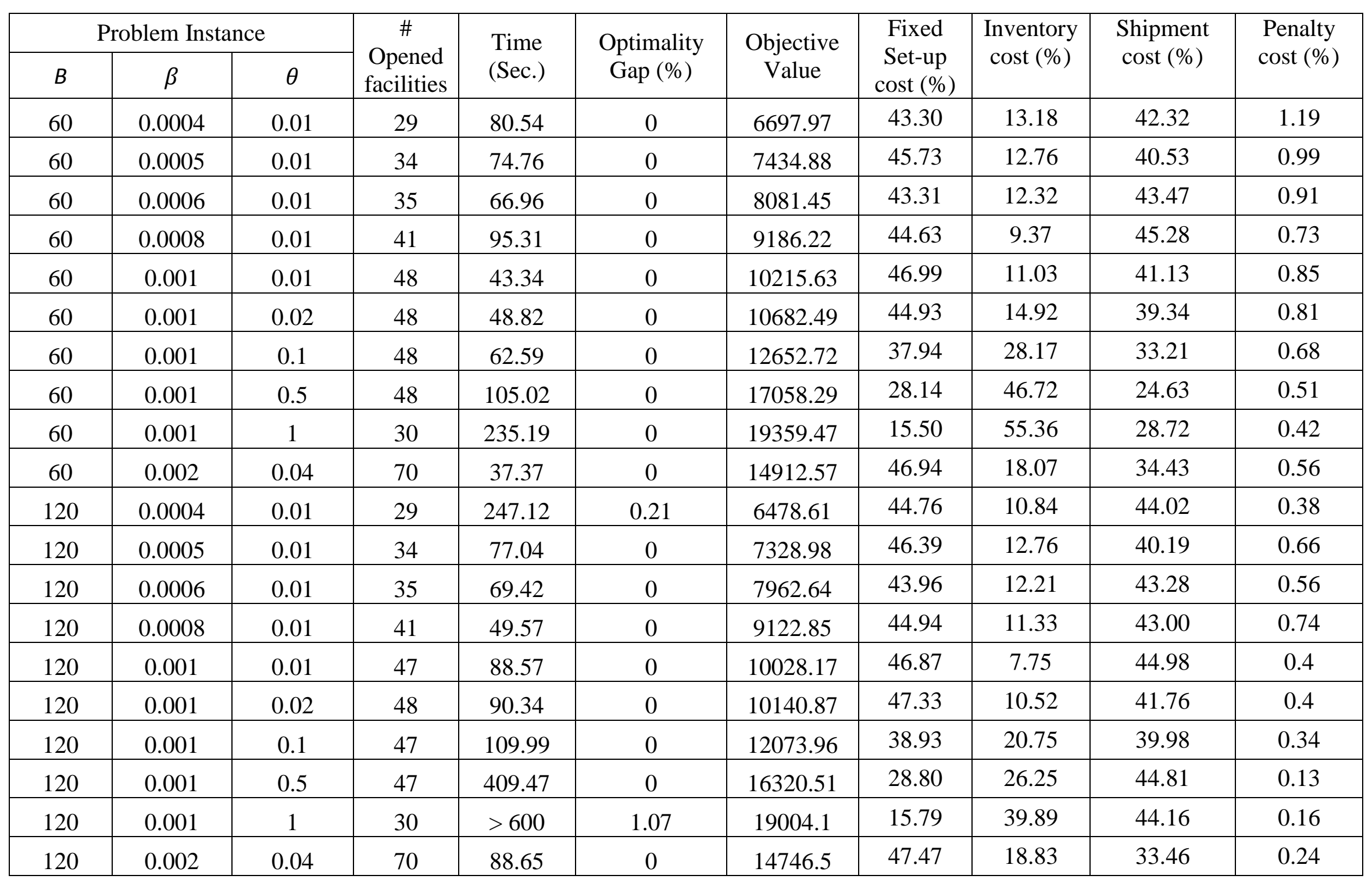


Table 5.13.c. LP/NLP-BB computational performance for 150-node problems, $q=0.05, R=4$

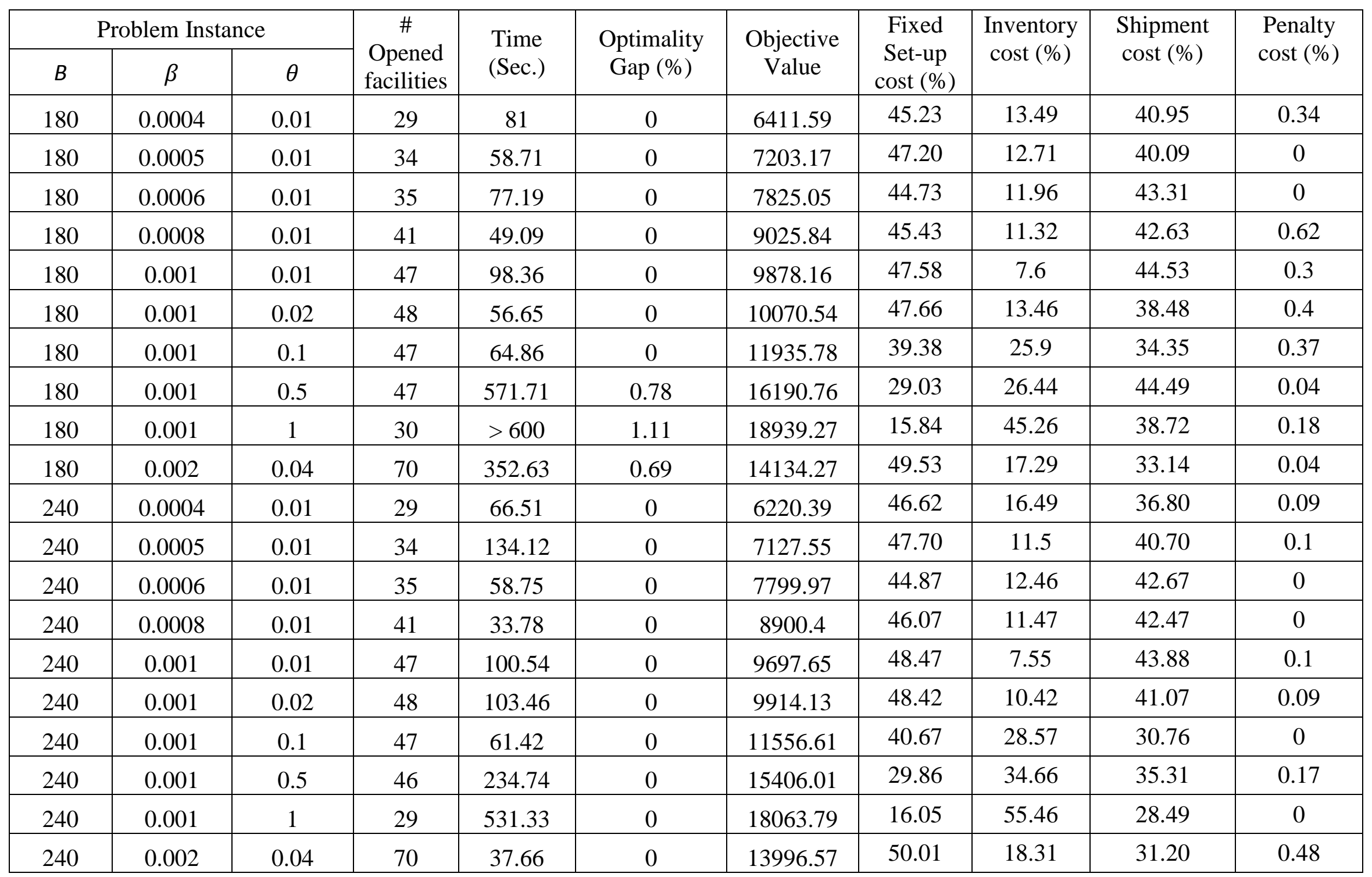


Table 5.13.d. LP/NLP-BB computational performance for 150-node problems, $q=0.05, R=4$

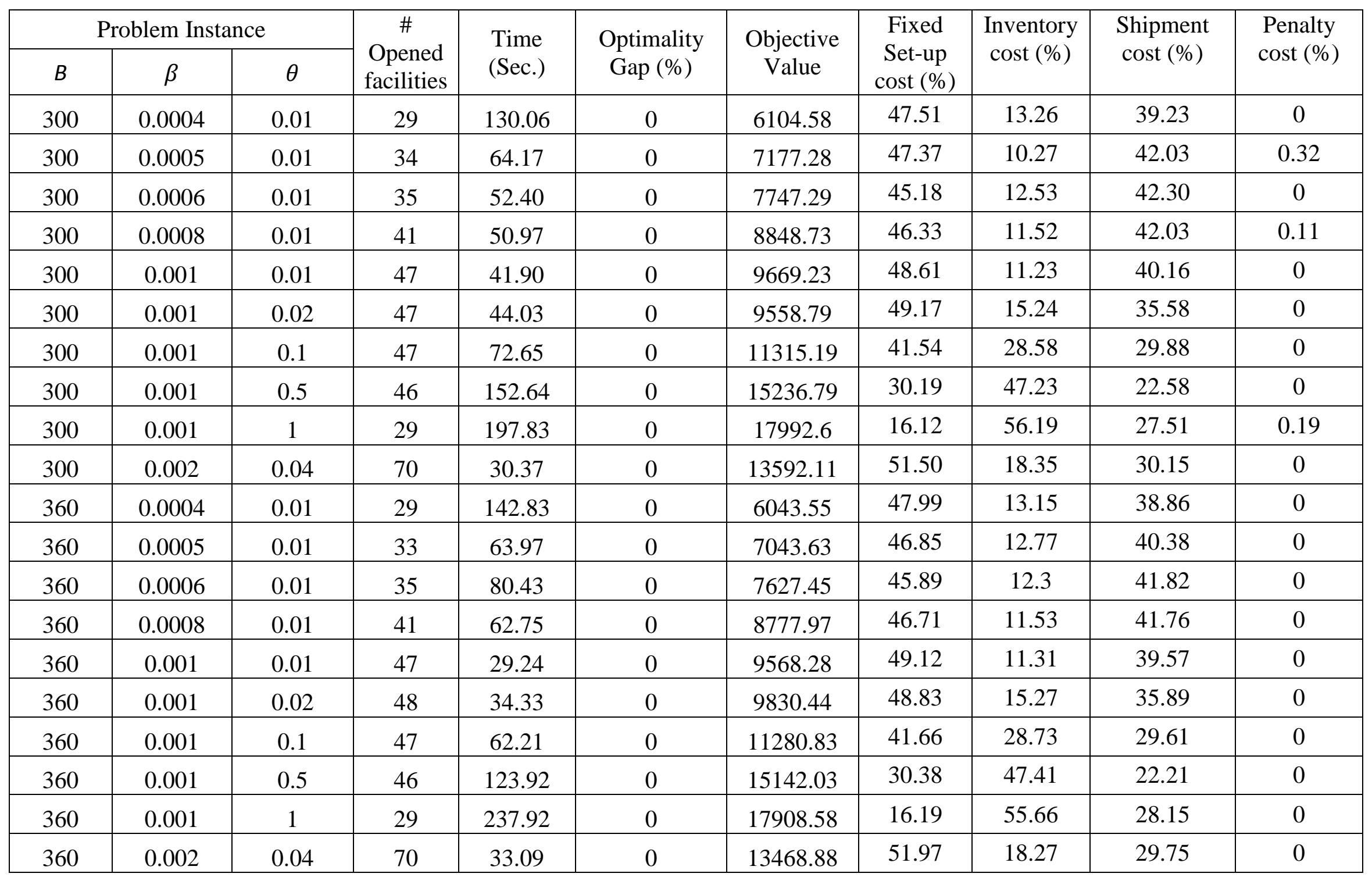


Table 5.14.a. LP/NLP-BB computational performance for 150-node problems, $q=0.05, R=5$

\begin{tabular}{|c|c|c|c|c|c|c|c|c|c|c|}
\hline \multicolumn{3}{|c|}{ Problem Instance } & \multirow{2}{*}{$\begin{array}{c}\# \\
\text { Opened } \\
\text { facilities } \\
\end{array}$} & \multirow{2}{*}{$\begin{array}{l}\text { Time } \\
\text { (Sec.) }\end{array}$} & \multirow{2}{*}{$\begin{array}{c}\text { Optimality } \\
\text { Gap (\%) }\end{array}$} & \multirow{2}{*}{$\begin{array}{c}\text { Objective } \\
\text { Value }\end{array}$} & \multirow{2}{*}{$\begin{array}{c}\text { Fixed } \\
\text { Set-up } \\
\text { cost }(\%)\end{array}$} & \multirow{2}{*}{$\begin{array}{c}\text { Inventory } \\
\text { cost }(\%)\end{array}$} & \multirow{2}{*}{$\begin{array}{l}\text { Shipment } \\
\text { cost }(\%)\end{array}$} & \multirow{2}{*}{$\begin{array}{l}\text { Penalty } \\
\text { cost }(\%)\end{array}$} \\
\hline$B$ & $\beta$ & $\theta$ & & & & & & & & \\
\hline 0 & 0.0004 & 0.01 & 29 & 27.99 & 0.16 & 6739.23 & 43.03 & 13.24 & 43.66 & 0.07 \\
\hline 0 & 0.0005 & 0.01 & 32 & 16.01 & 0 & 7609.44 & 42.05 & 12.34 & 45.54 & 0.06 \\
\hline 0 & 0.0006 & 0.01 & 32 & 16.33 & 0.05 & 8126.85 & 39.38 & 11.52 & 49.04 & 0.06 \\
\hline 0 & 0.0008 & 0.01 & 41 & 14.14 & 0.11 & 9461.63 & 43.33 & 10.97 & 45.65 & 0.05 \\
\hline 0 & 0.001 & 0.01 & 48 & 5.20 & 0 & 10226.89 & 46.94 & 11.03 & 42.04 & 0 \\
\hline 0 & 0.001 & 0.02 & 48 & 6.59 & 0 & 10694.12 & 44.88 & 14.92 & 40.20 & 0 \\
\hline 0 & 0.001 & 0.1 & 48 & 10.59 & 0 & 12665.92 & 37.90 & 28.16 & 33.94 & 0 \\
\hline 0 & 0.001 & 0.5 & 48 & 53.92 & 0.44 & 17075.01 & 28.11 & 46.71 & 25.18 & 0 \\
\hline 0 & 0.001 & 1 & 30 & 229.01 & 0.85 & 19721.18 & 15.21 & 44.93 & 39.83 & 0.02 \\
\hline 0 & 0.002 & 0.04 & 70 & 3.64 & 0 & 14995.24 & 46.68 & 18.2 & 35.12 & 0 \\
\hline 30 & 0.0004 & 0.01 & 29 & 109.86 & 0 & 6763.9 & 42.87 & 13.2 & 43.87 & 0.05 \\
\hline 30 & 0.0005 & 0.01 & 34 & 103.13 & 0 & 7418.44 & 45.83 & 12.99 & 41.13 & 0.06 \\
\hline 30 & 0.0006 & 0.01 & 36 & 83.14 & 0 & 8048 & 44.73 & 12.35 & 42.86 & 0.05 \\
\hline 30 & 0.0008 & 0.01 & 41 & 61.52 & 0 & 9193.22 & 44.60 & 11.42 & 43.93 & 0.05 \\
\hline 30 & 0.001 & 0.01 & 48 & 49.67 & 0 & 10181.41 & 47.14 & 11.07 & 41.74 & 0.04 \\
\hline 30 & 0.001 & 0.02 & 48 & 56.33 & 0 & 10648.39 & 45.08 & 14.97 & 39.91 & 0.04 \\
\hline 30 & 0.001 & 0.1 & 48 & 69.03 & 0 & 12619.15 & 38.04 & 28.25 & 33.68 & 0.03 \\
\hline 30 & 0.001 & 0.5 & 48 & 158.24 & 0 & 17025.92 & 28.19 & 46.82 & 24.96 & 0.03 \\
\hline 30 & 0.001 & 1 & 30 & 477.92 & 0 & 19654.21 & 15.26 & 55.46 & 29.26 & 0.02 \\
\hline 30 & 0.002 & 0.04 & 70 & 40.36 & 0 & 14926.39 & 46.90 & 18.27 & 34.80 & 0.03 \\
\hline
\end{tabular}


Table 5.14.b. LP/NLP-BB computational performance for 150-node problems, $q=0.05, R=5$

\begin{tabular}{|c|c|c|c|c|c|c|c|c|c|c|}
\hline \multicolumn{3}{|c|}{ Problem Instance } & \multirow{2}{*}{$\begin{array}{c}\text { \# } \\
\text { Opened } \\
\text { facilities }\end{array}$} & \multirow{2}{*}{$\begin{array}{l}\text { Time } \\
(\text { Sec. })\end{array}$} & \multirow{2}{*}{$\begin{array}{c}\text { Optimality } \\
\text { Gap }(\%)\end{array}$} & \multirow{2}{*}{$\begin{array}{c}\text { Objective } \\
\text { Value }\end{array}$} & \multirow{2}{*}{$\begin{array}{c}\text { Fixed } \\
\text { Set-up } \\
\text { cost }(\%)\end{array}$} & \multirow{2}{*}{$\begin{array}{l}\text { Inventory } \\
\text { cost }(\%)\end{array}$} & \multirow{2}{*}{$\begin{array}{l}\text { Shipment } \\
\text { cost }(\%)\end{array}$} & \multirow{2}{*}{$\begin{array}{l}\text { Penalty } \\
\text { cost }(\%)\end{array}$} \\
\hline$B$ & $\beta$ & $\theta$ & & & & & & & & \\
\hline 60 & 0.0004 & 0.01 & 29 & 107.38 & 0 & 6621.82 & 43.79 & 13.33 & 42.82 & 0.06 \\
\hline 60 & 0.0005 & 0.01 & 34 & 101.17 & 0 & 7364.67 & 46.17 & 12.88 & 40.91 & 0.04 \\
\hline 60 & 0.0006 & 0.01 & 36 & 87.30 & 0 & 8011.66 & 44.93 & 12.42 & 42.60 & 0.05 \\
\hline 60 & 0.0008 & 0.01 & 41 & 134.51 & 0 & 9068.88 & 45.21 & 9.21 & 45.56 & 0.03 \\
\hline 60 & 0.001 & 0.01 & 48 & 50.97 & 0 & 10133.72 & 47.37 & 11.12 & 41.47 & 0.04 \\
\hline 60 & 0.001 & 0.02 & 48 & 56.05 & 0 & 10600.57 & 45.28 & 15.04 & 39.64 & 0.04 \\
\hline 60 & 0.001 & 0.1 & 48 & 68.75 & 0 & 12570.81 & 38.18 & 28.35 & 33.43 & 0.03 \\
\hline 60 & 0.001 & 0.5 & 48 & 164.89 & 0 & 16976.4 & 28.27 & 46.95 & 24.75 & 0.03 \\
\hline 60 & 0.001 & 1 & 30 & 436.39 & 0 & 19577.59 & 15.32 & 55.58 & 29.07 & 0.02 \\
\hline 60 & 0.002 & 0.04 & 70 & 43.24 & 0 & 14833.53 & 47.19 & 18.16 & 34.62 & 0.03 \\
\hline 120 & 0.0004 & 0.01 & 29 & 280.15 & 0.81 & 6541.44 & 44.33 & 11.78 & 43.86 & 0.03 \\
\hline 120 & 0.0005 & 0.01 & 34 & 105.16 & 0 & 7282.93 & 46.68 & 12.85 & 40.45 & 0.03 \\
\hline 120 & 0.0006 & 0.01 & 36 & 75.75 & 0 & 8071.77 & 44.60 & 12.14 & 43.24 & 0.03 \\
\hline 120 & 0.0008 & 0.01 & 41 & 57.60 & 0 & 9058.63 & 45.26 & 11.41 & 43.30 & 0.03 \\
\hline 120 & 0.001 & 0.01 & 48 & 116.11 & 0 & 10182.82 & 47.14 & 17.78 & 35.06 & 0.02 \\
\hline 120 & 0.001 & 0.02 & 48 & 119.89 & 0 & 10495.13 & 45.74 & 10.56 & 43.68 & 0.02 \\
\hline 120 & 0.001 & 0.1 & 48 & 524.78 & 0.11 & 11879.83 & 40.40 & 14.25 & 45.34 & 0.01 \\
\hline 120 & 0.001 & 0.5 & 48 & 196.93 & 0 & 17010.75 & 28.22 & 36.74 & 35.02 & 0.01 \\
\hline 120 & 0.001 & 1 & 30 & $>600$ & 1.14 & 19550.85 & 15.42 & 44.21 & 40.35 & 0.01 \\
\hline 120 & 0.002 & 0.04 & 70 & 112.96 & 0 & 14750.84 & 47.45 & 9.85 & 42.69 & 0.01 \\
\hline
\end{tabular}


Table 5.14.c. LP/NLP-BB computational performance for 150-node problems, $q=0.05, R=5$

\begin{tabular}{|c|c|c|c|c|c|c|c|c|c|c|}
\hline \multicolumn{3}{|c|}{ Problem Instance } & \multirow{2}{*}{$\begin{array}{c}\# \\
\text { Opened } \\
\text { facilities }\end{array}$} & \multirow{2}{*}{$\begin{array}{l}\text { Time } \\
\text { (Sec.) }\end{array}$} & \multirow{2}{*}{$\begin{array}{c}\text { Optimality } \\
\text { Gap }(\%)\end{array}$} & \multirow{2}{*}{$\begin{array}{c}\text { Objective } \\
\text { Value }\end{array}$} & \multirow{2}{*}{$\begin{array}{c}\text { Fixed } \\
\text { Set-up } \\
\text { cost }(\%)\end{array}$} & \multirow{2}{*}{$\begin{array}{l}\text { Inventory } \\
\text { cost }(\%)\end{array}$} & \multirow{2}{*}{$\begin{array}{c}\text { Shipment } \\
\text { cost }(\%)\end{array}$} & \multirow{2}{*}{$\begin{array}{l}\text { Penalty } \\
\text { cost }(\%)\end{array}$} \\
\hline$B$ & $\beta$ & $\theta$ & & & & & & & & \\
\hline 180 & 0.0004 & 0.01 & 28 & 90.84 & 0 & 6490.57 & 43.14 & 13.53 & 43.32 & 0.01 \\
\hline 180 & 0.0005 & 0.01 & 34 & 92.02 & 0 & 7203.59 & 47.20 & 12.71 & 40.06 & 0.02 \\
\hline 180 & 0.0006 & 0.01 & 36 & 321.00 & 0 & 8084.42 & 44.53 & 8.31 & 47.15 & 0.01 \\
\hline 180 & 0.0008 & 0.01 & 41 & 57.56 & 0 & 8972.69 & 45.69 & 11.39 & 42.89 & 0.03 \\
\hline 180 & 0.001 & 0.01 & 48 & 102.48 & 0 & 10146.81 & 47.31 & 7.62 & 45.05 & 0.02 \\
\hline 180 & 0.001 & 0.02 & 48 & 67.91 & 0 & 10344.76 & 46.40 & 13.49 & 40.09 & 0.02 \\
\hline 180 & 0.001 & 0.1 & 48 & 90.81 & 0 & 11619.5 & 41.31 & 25.94 & 32.74 & 0.02 \\
\hline 180 & 0.001 & 0.5 & 48 & 554.68 & 0.45 & 16928.42 & 28.35 & 36.46 & 35.18 & 0.02 \\
\hline 180 & 0.001 & 1 & 29 & 432.50 & 0 & 19089.62 & 15.19 & 55.94 & 28.87 & 0 \\
\hline 180 & 0.002 & 0.04 & 70 & 353.33 & 0.43 & 14809.91 & 47.27 & 16.46 & 36.27 & 0 \\
\hline 240 & 0.0004 & 0.01 & 29 & 100.28 & 0.71 & 6295.57 & 46.06 & 10.79 & 43.15 & 0 \\
\hline 240 & 0.0005 & 0.01 & 34 & 168.01 & 0 & 7159 & 47.49 & 11.68 & 40.83 & 0 \\
\hline 240 & 0.0006 & 0.01 & 36 & 83.31 & 0 & 7800.83 & 46.15 & 12.46 & 41.38 & 0.02 \\
\hline 240 & 0.0008 & 0.01 & 41 & 57.09 & 0 & 8901.08 & 46.06 & 11.47 & 42.46 & 0.01 \\
\hline 240 & 0.001 & 0.01 & 48 & 114.25 & 0 & 10085.48 & 47.59 & 7.79 & 44.62 & 0 \\
\hline 240 & 0.001 & 0.02 & 48 & 128.23 & 0 & 10272.12 & 46.73 & 10.69 & 42.58 & 0 \\
\hline 240 & 0.001 & 0.1 & 48 & 147.33 & 0 & 11549.71 & 41.56 & 21.07 & 37.36 & 0 \\
\hline 240 & 0.001 & 0.5 & 49 & $>600$ & 1.21 & 17654.03 & 27.76 & 29.33 & 42.90 & 3.89 \\
\hline 240 & 0.001 & 1 & 33 & $>600$ & 1.01 & 19321.2 & 17.08 & 43.88 & 39.04 & 2.54 \\
\hline 240 & 0.002 & 0.04 & 70 & 40.37 & 0 & 14530.11 & 48.18 & 18.4 & 33.40 & 0.02 \\
\hline
\end{tabular}


Table 5.14.d. LP/NLP-BB computational performance for 150-node problems, $q=0.05, R=5$

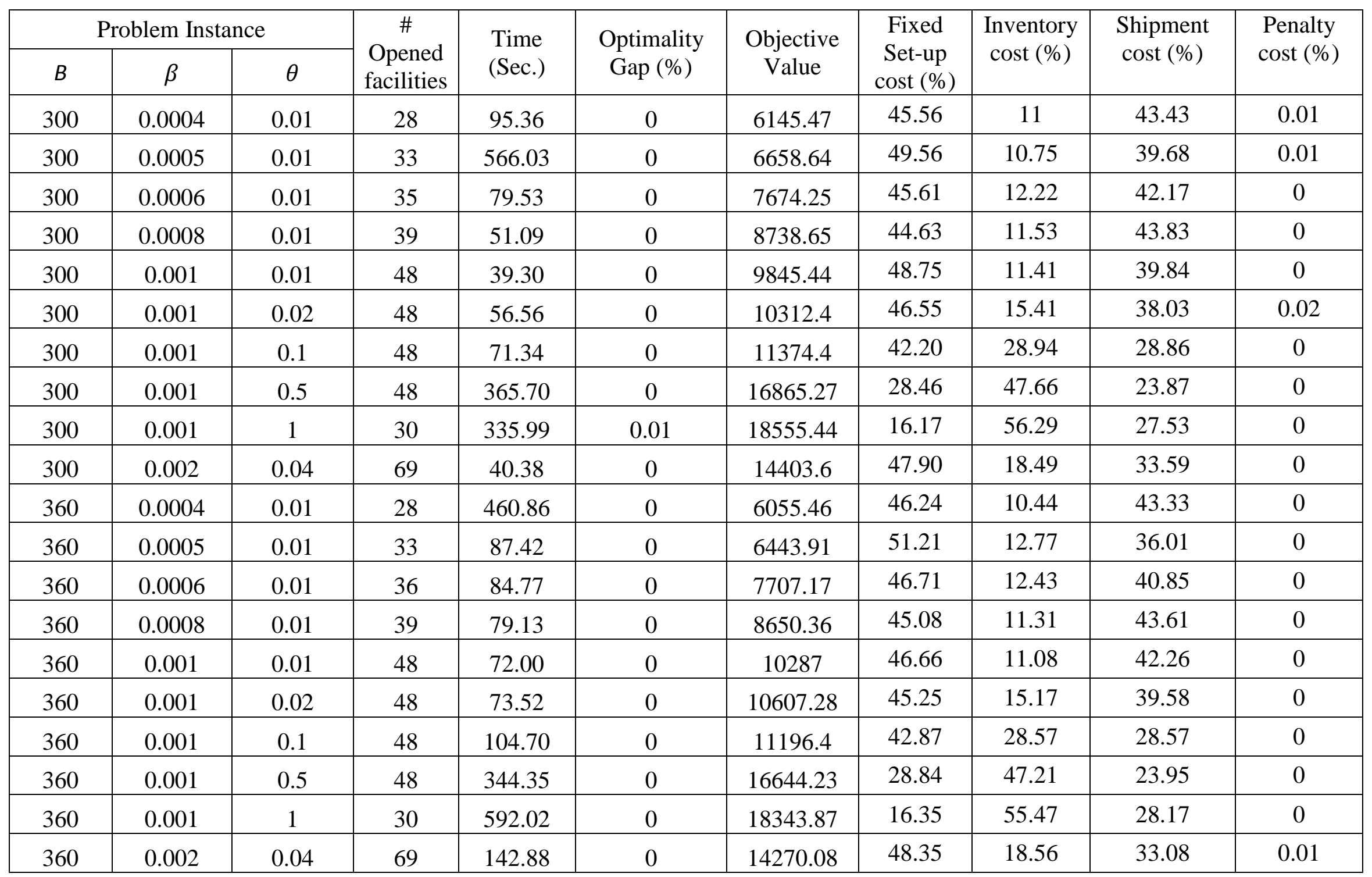


Table 5.15.a. LP/NLP-BB computational performance for 150-node problems, $q=0.01, R=3$

\begin{tabular}{|c|c|c|c|c|c|c|c|c|c|c|}
\hline \multicolumn{3}{|c|}{ Problem Instance } & \multirow{2}{*}{$\begin{array}{c}\# \\
\text { Opened } \\
\text { facilities }\end{array}$} & \multirow{2}{*}{$\begin{array}{l}\text { Time } \\
\text { (Sec.) }\end{array}$} & \multirow{2}{*}{$\begin{array}{c}\text { Optimality } \\
\text { Gap }(\%)\end{array}$} & \multirow{2}{*}{$\begin{array}{c}\text { Objective } \\
\text { Value }\end{array}$} & \multirow{2}{*}{$\begin{array}{c}\text { Fixed } \\
\text { Set-up } \\
\text { cost }(\%)\end{array}$} & \multirow{2}{*}{$\begin{array}{c}\text { Inventory } \\
\text { cost }(\%)\end{array}$} & \multirow{2}{*}{$\begin{array}{c}\text { Shipment } \\
\text { cost }(\%)\end{array}$} & \multirow{2}{*}{$\begin{array}{l}\text { Penalty } \\
\text { cost }(\%)\end{array}$} \\
\hline$B$ & $\beta$ & $\theta$ & & & & & & & & \\
\hline 0 & 0.0004 & 0.01 & 28 & 9.58 & 0.54 & 6323.82 & 44.28 & 13.91 & 41.81 & 0 \\
\hline 0 & 0.0005 & 0.01 & 31 & 8.44 & 0.05 & 7000.56 & 44.28 & 12.57 & 42.94 & 0.22 \\
\hline 0 & 0.0006 & 0.01 & 34 & 7.03 & 0.25 & 7645.99 & 44.47 & 11.51 & 44.02 & 0 \\
\hline 0 & 0.0008 & 0.01 & 39 & 3.37 & 0 & 8790.99 & 44.36 & 11.61 & 43.85 & 0.18 \\
\hline 0 & 0.001 & 0.01 & 47 & 2.72 & 0 & 9557.14 & 49.18 & 11.57 & 39.25 & 0 \\
\hline 0 & 0.001 & 0.02 & 47 & 3.26 & 0 & 10344.83 & 45.43 & 15 & 39.42 & 0.15 \\
\hline 0 & 0.001 & 0.1 & 47 & 6.06 & 0 & 11948.1 & 39.34 & 29.27 & 31.40 & 0 \\
\hline 0 & 0.001 & 0.5 & 47 & 10.73 & 0.73 & 15637.75 & 30.06 & 39.5 & 30.34 & 0.1 \\
\hline 0 & 0.001 & 1 & 28 & 20.42 & 0 & 18875.82 & 14.83 & 46.48 & 38.60 & 0.08 \\
\hline 0 & 0.002 & 0.04 & 66 & 2.12 & 0 & 13936.73 & 47.36 & 18.72 & 33.92 & 0 \\
\hline 30 & 0.0004 & 0.01 & 28 & 63.28 & 0 & 6326.99 & 44.25 & 13.9 & 41.60 & 0.24 \\
\hline 30 & 0.0005 & 0.01 & 31 & 56.48 & 0 & 6997.08 & 44.30 & 13.13 & 42.35 & 0.21 \\
\hline 30 & 0.0006 & 0.01 & 35 & 49.84 & 0 & 7604.65 & 46.02 & 12.79 & 41.00 & 0.18 \\
\hline 30 & 0.0008 & 0.01 & 38 & 39.87 & 0 & 8632.43 & 44.02 & 11.72 & 44.10 & 0.16 \\
\hline 30 & 0.001 & 0.01 & 47 & 37.06 & 0 & 9561.45 & 49.16 & 11.56 & 39.13 & 0.15 \\
\hline 30 & 0.001 & 0.02 & 47 & 39.54 & 0 & 10219.42 & 45.99 & 15.61 & 38.26 & 0.14 \\
\hline 30 & 0.001 & 0.1 & 47 & 48.95 & 0 & 11902.15 & 39.49 & 29.25 & 31.14 & 0.12 \\
\hline 30 & 0.001 & 0.5 & 47 & 87.46 & 0 & 15573.85 & 30.18 & 48.04 & 21.69 & 0.09 \\
\hline 30 & 0.001 & 1 & 29 & 85.15 & 0.39 & 18920.18 & 15.33 & 49.81 & 34.79 & 0.07 \\
\hline 30 & 0.002 & 0.04 & 66 & 26.74 & 0 & 13921.16 & 47.41 & 18.74 & 33.85 & 0 \\
\hline
\end{tabular}


Table 5.15.b. LP/NLP-BB computational performance for 150-node problems, $q=0.01, R=3$

\begin{tabular}{|c|c|c|c|c|c|c|c|c|c|c|}
\hline \multicolumn{3}{|c|}{ Problem Instance } & \multirow{2}{*}{$\begin{array}{c}\# \\
\text { Opened } \\
\text { facilities }\end{array}$} & \multirow{2}{*}{$\begin{array}{l}\text { Time } \\
\text { (Sec.) }\end{array}$} & \multirow{2}{*}{$\begin{array}{c}\text { Optimality } \\
\text { Gap (\%) }\end{array}$} & \multirow{2}{*}{$\begin{array}{c}\text { Objective } \\
\text { Value }\end{array}$} & \multirow{2}{*}{$\begin{array}{c}\text { Fixed } \\
\text { Set-up } \\
\text { cost }(\%)\end{array}$} & \multirow{2}{*}{$\begin{array}{c}\text { Inventory } \\
\text { cost }(\%)\end{array}$} & \multirow{2}{*}{$\begin{array}{l}\text { Shipment } \\
\text { cost }(\%)\end{array}$} & \multirow{2}{*}{$\begin{array}{l}\text { Penalty } \\
\text { cost }(\%)\end{array}$} \\
\hline$B$ & $\beta$ & $\theta$ & & & & & & & & \\
\hline 60 & 0.0004 & 0.01 & 28 & 61.68 & 0 & 6315.01 & 44.34 & 13.93 & 41.51 & 0.22 \\
\hline 60 & 0.0005 & 0.01 & 31 & 50.48 & 0 & 6984.66 & 44.38 & 13.15 & 42.27 & 0.19 \\
\hline 60 & 0.0006 & 0.01 & 35 & 44.06 & 0 & 7594.6 & 46.09 & 12.81 & 40.94 & 0.16 \\
\hline 60 & 0.0008 & 0.01 & 38 & 32.87 & 0 & 8611.72 & 44.13 & 11.74 & 44.13 & 0 \\
\hline 60 & 0.001 & 0.01 & 47 & 37.59 & 0 & 9551.75 & 49.21 & 11.57 & 39.07 & 0.14 \\
\hline 60 & 0.001 & 0.02 & 47 & 37.50 & 0 & 10109.7 & 46.49 & 15.62 & 37.75 & 0.14 \\
\hline 60 & 0.001 & 0.1 & 47 & 46.50 & 0 & 11895.32 & 39.51 & 29.28 & 31.10 & 0.12 \\
\hline 60 & 0.001 & 0.5 & 47 & 85.32 & 0 & 15493.79 & 30.33 & 48.07 & 21.52 & 0.09 \\
\hline 60 & 0.001 & 1 & 27 & 120.86 & 0 & 18709.82 & 14.43 & 44.73 & 40.78 & 0.06 \\
\hline 60 & 0.002 & 0.04 & 66 & 27.95 & 0 & 13906.57 & 47.46 & 18.76 & 33.78 & 0 \\
\hline 120 & 0.0004 & 0.01 & 28 & 67.85 & 0 & 6296.09 & 44.47 & 13.97 & 41.41 & 0.16 \\
\hline 120 & 0.0005 & 0.01 & 30 & 55.18 & 0 & 6946.57 & 43.19 & 12.98 & 43.69 & 0.14 \\
\hline 120 & 0.0006 & 0.01 & 35 & 26.87 & 0 & 7568.92 & 46.24 & 12.85 & 40.91 & 0 \\
\hline 120 & 0.0008 & 0.01 & 38 & 40.51 & 0 & 8600.6 & 44.18 & 11.76 & 43.94 & 0.12 \\
\hline 120 & 0.001 & 0.01 & 47 & 26.40 & 0 & 9523.54 & 49.35 & 11.6 & 39.04 & 0 \\
\hline 120 & 0.001 & 0.02 & 47 & 26.65 & 0 & 9981.3 & 47.09 & 15.66 & 37.25 & 0 \\
\hline 120 & 0.001 & 0.1 & 47 & 35.49 & 0 & 11813.12 & 39.79 & 29.33 & 30.87 & 0 \\
\hline 120 & 0.001 & 0.5 & 47 & 71.90 & 0 & 15332.81 & 30.65 & 48.14 & 21.21 & 0 \\
\hline 120 & 0.001 & 1 & 26 & 98.82 & 0 & 18550.15 & 14.02 & 44.51 & 41.43 & 0.05 \\
\hline 120 & 0.002 & 0.04 & 66 & 20.59 & 0 & 13882.88 & 47.54 & 18.78 & 33.68 & 0 \\
\hline
\end{tabular}


Table 5.15.c. LP/NLP-BB computational performance for 150-node problems, $q=0.01, R=3$

\begin{tabular}{|c|c|c|c|c|c|c|c|c|c|c|}
\hline \multicolumn{3}{|c|}{ Problem Instance } & \multirow{2}{*}{$\begin{array}{c}\# \\
\text { Opened } \\
\text { facilities }\end{array}$} & \multirow{2}{*}{$\begin{array}{l}\text { Time } \\
\text { (Sec.) }\end{array}$} & \multirow{2}{*}{$\begin{array}{c}\text { Optimality } \\
\text { Gap (\%) }\end{array}$} & \multirow{2}{*}{$\begin{array}{c}\text { Objective } \\
\text { Value }\end{array}$} & \multirow{2}{*}{$\begin{array}{c}\text { Fixed } \\
\text { Set-up } \\
\text { cost }(\%)\end{array}$} & \multirow{2}{*}{$\begin{array}{l}\text { Inventory } \\
\text { cost }(\%)\end{array}$} & \multirow{2}{*}{$\begin{array}{l}\text { Shipment } \\
\text { cost }(\%)\end{array}$} & \multirow{2}{*}{$\begin{array}{l}\text { Penalty } \\
\text { cost }(\%)\end{array}$} \\
\hline$B$ & $\beta$ & $\theta$ & & & & & & & & \\
\hline 180 & 0.0004 & 0.01 & 28 & 42.01 & 0.16 & 6276.11 & 44.61 & 14.01 & 41.38 & 0 \\
\hline 180 & 0.0005 & 0.01 & 30 & 33.32 & 0 & 6925.03 & 43.32 & 13.02 & 43.66 & 0 \\
\hline 180 & 0.0006 & 0.01 & 35 & 14.71 & 0 & 7534.67 & 46.45 & 11.61 & 41.95 & 0 \\
\hline 180 & 0.0008 & 0.01 & 38 & 26.86 & 0 & 8576.46 & 44.31 & 11.79 & 43.91 & 0 \\
\hline 180 & 0.001 & 0.01 & 47 & 24.25 & 0 & 9485.45 & 49.55 & 11.62 & 38.83 & 0 \\
\hline 180 & 0.001 & 0.02 & 47 & 23.92 & 0 & 9963.14 & 47.17 & 15.68 & 37.14 & 0 \\
\hline 180 & 0.001 & 0.1 & 47 & 31.01 & 0 & 11794.65 & 39.85 & 29.38 & 30.77 & 0 \\
\hline 180 & 0.001 & 0.5 & 47 & 86.97 & 0 & 15273.65 & 30.77 & 48.19 & 21.04 & 0 \\
\hline 180 & 0.001 & 1 & 26 & 139.32 & 0 & 18490.09 & 14.06 & 49.78 & 36.13 & 0.03 \\
\hline 180 & 0.002 & 0.04 & 66 & 15.68 & 0 & 13825.32 & 47.74 & 18.8 & 33.46 & 0 \\
\hline 240 & 0.0004 & 0.01 & 28 & 43.46 & 0.17 & 6267.39 & 44.68 & 14.03 & 41.30 & 0 \\
\hline 240 & 0.0005 & 0.01 & 30 & 36.97 & 0 & 6914.52 & 43.39 & 13.03 & 43.58 & 0 \\
\hline 240 & 0.0006 & 0.01 & 35 & 17.17 & 0 & 7561.66 & 46.29 & 11.63 & 42.09 & 0 \\
\hline 240 & 0.0008 & 0.01 & 38 & 27.17 & 0 & 8561.77 & 44.38 & 11.8 & 43.81 & 0 \\
\hline 240 & 0.001 & 0.01 & 47 & 16.47 & 0 & 9451.36 & 49.73 & 11.64 & 38.63 & 0 \\
\hline 240 & 0.001 & 0.02 & 47 & 16.48 & 0 & 9948.89 & 47.24 & 15.7 & 37.06 & 0 \\
\hline 240 & 0.001 & 0.1 & 47 & 23.81 & 0 & 11679.75 & 40.24 & 29.4 & 30.35 & 0 \\
\hline 240 & 0.001 & 0.5 & 44 & 81.97 & 0 & 15097.28 & 29.14 & 48.22 & 22.64 & 0 \\
\hline 240 & 0.001 & 1 & 25 & 138.47 & 0 & 18298.48 & 13.66 & 50.06 & 36.26 & 0.02 \\
\hline 240 & 0.002 & 0.04 & 66 & 17.76 & 0 & 13846.09 & 47.67 & 18.83 & 33.51 & 0 \\
\hline
\end{tabular}


Table 5.15.d. LP/NLP-BB computational performance for 150-node problems, $q=0.01, R=3$

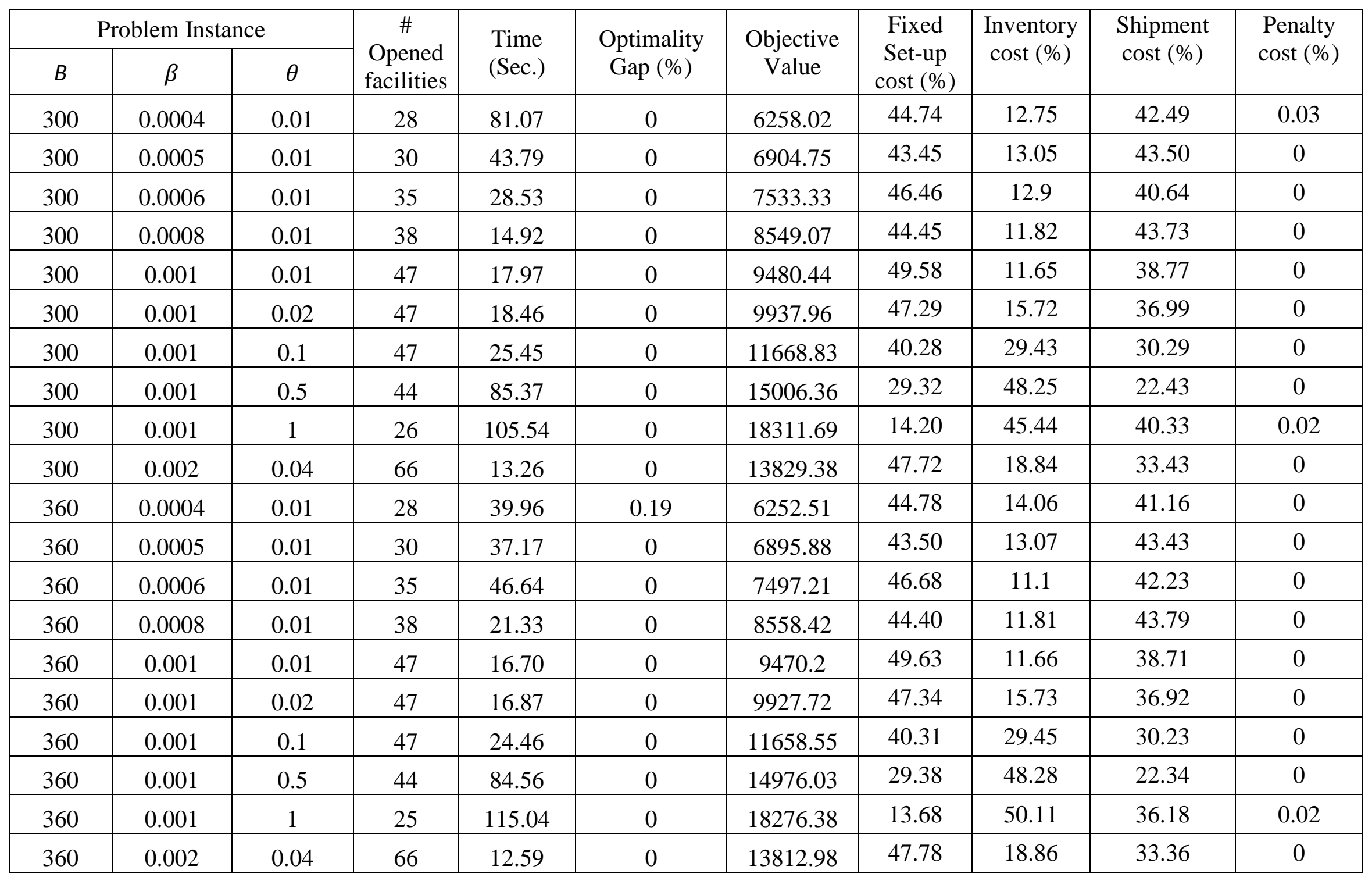


Table 5.16.a. LP/NLP-BB computational performance for 150-node problems, $q=0.03, R=3$

\begin{tabular}{|c|c|c|c|c|c|c|c|c|c|c|}
\hline \multicolumn{3}{|c|}{ Problem Instance } & \multirow{2}{*}{$\begin{array}{c}\# \\
\text { Opened } \\
\text { facilities }\end{array}$} & \multirow{2}{*}{$\begin{array}{l}\text { Time } \\
\text { (Sec.) }\end{array}$} & \multirow{2}{*}{$\begin{array}{c}\text { Optimality } \\
\text { Gap }(\%)\end{array}$} & \multirow{2}{*}{$\begin{array}{c}\text { Objective } \\
\text { Value }\end{array}$} & \multirow{2}{*}{$\begin{array}{c}\text { Fixed } \\
\text { Set-up } \\
\text { cost }(\%)\end{array}$} & \multirow{2}{*}{$\begin{array}{c}\text { Inventory } \\
\text { cost }(\%)\end{array}$} & \multirow{2}{*}{$\begin{array}{c}\text { Shipment } \\
\text { cost }(\%)\end{array}$} & \multirow{2}{*}{$\begin{array}{l}\text { Penalty } \\
\text { cost }(\%)\end{array}$} \\
\hline$B$ & $\beta$ & $\theta$ & & & & & & & & \\
\hline 0 & 0.0004 & 0.01 & 29 & 11.19 & 0.16 & 7481.15 & 38.76 & 12.4 & 28.6 & 20.23 \\
\hline 0 & 0.0005 & 0.01 & 31 & 9.24 & 0.08 & 9187.36 & 33.74 & 11.92 & 35.81 & 18.52 \\
\hline 0 & 0.0006 & 0.01 & 35 & 10.25 & 0.17 & 9868.32 & 35.47 & 11.27 & 36.13 & 17.12 \\
\hline 0 & 0.0008 & 0.01 & 41 & 3.52 & 0 & 10281.29 & 39.88 & 11.4 & 33.67 & 15.05 \\
\hline 0 & 0.001 & 0.01 & 50 & 7.27 & 0.03 & 12116.66 & 41.27 & 9.49 & 36.14 & 13.1 \\
\hline 0 & 0.001 & 0.02 & 51 & 2.97 & 0 & 11505.93 & 44.32 & 14.91 & 27.76 & 13.01 \\
\hline 0 & 0.001 & 0.1 & 50 & 10.34 & 0.42 & 14289.72 & 34.99 & 22.19 & 31.81 & 11.01 \\
\hline 0 & 0.001 & 0.5 & 49 & 19.03 & 0.68 & 19005.16 & 25.78 & 37.39 & 28.43 & 8.41 \\
\hline 0 & 0.001 & 1 & 30 & 99.36 & 0.91 & 22489.33 & 13.34 & 44.76 & 34.83 & 7.08 \\
\hline 0 & 0.002 & 0.04 & 70 & 3.24 & 0 & 17423.55 & 40.18 & 21.21 & 28.8 & 9.81 \\
\hline 30 & 0.0004 & 0.01 & 30 & 50.22 & 0 & 7398.61 & 40.55 & 12.85 & 28.21 & 18.38 \\
\hline 30 & 0.0005 & 0.01 & 35 & 63.61 & 0 & 9174.82 & 38.15 & 12.61 & 32.13 & 17.11 \\
\hline 30 & 0.0006 & 0.01 & 34 & 54.01 & 0 & 9784.04 & 34.75 & 12.25 & 37.12 & 15.88 \\
\hline 30 & 0.0008 & 0.01 & 41 & 42.45 & 0 & 10248.54 & 40.01 & 11.7 & 34.29 & 14 \\
\hline 30 & 0.001 & 0.01 & 48 & 37.41 & 0 & 11956.98 & 40.14 & 11.55 & 35.62 & 12.69 \\
\hline 30 & 0.001 & 0.02 & 48 & 39.66 & 0 & 11416.11 & 42.05 & 14.96 & 30.86 & 12.13 \\
\hline 30 & 0.001 & 0.1 & 48 & 49.21 & 0 & 14316.81 & 33.53 & 26.91 & 29.39 & 10.18 \\
\hline 30 & 0.001 & 0.5 & 48 & 88.00 & 0 & 18903.32 & 25.39 & 44.55 & 22.76 & 7.3 \\
\hline 30 & 0.001 & 1 & 30 & 126.37 & 0 & 21889.12 & 13.71 & 43.6 & 36.68 & 6.01 \\
\hline 30 & 0.002 & 0.04 & 69 & 33.66 & 0 & 16990.91 & 40.61 & 18.39 & 32.17 & 8.83 \\
\hline
\end{tabular}


Table 5.16.b. LP/NLP-BB computational performance for 150-node problems, $q=0.03, R=3$

\begin{tabular}{|c|c|c|c|c|c|c|c|c|c|c|}
\hline \multicolumn{3}{|c|}{ Problem Instance } & \multirow{2}{*}{$\begin{array}{c}\# \\
\text { Opened } \\
\text { facilities }\end{array}$} & \multirow{2}{*}{$\begin{array}{l}\text { Time } \\
\text { (Sec.) }\end{array}$} & \multirow{2}{*}{$\begin{array}{c}\text { Optimality } \\
\text { Gap }(\%)\end{array}$} & \multirow{2}{*}{$\begin{array}{l}\text { Objective } \\
\text { Value }\end{array}$} & \multirow{2}{*}{$\begin{array}{c}\text { Fixed } \\
\text { Set-up } \\
\text { cost }(\%)\end{array}$} & \multirow{2}{*}{$\begin{array}{c}\text { Inventory } \\
\text { cost }(\%)\end{array}$} & \multirow{2}{*}{$\begin{array}{l}\text { Shipment } \\
\text { cost }(\%)\end{array}$} & \multirow{2}{*}{$\begin{array}{l}\text { Penalty } \\
\text { cost }(\%)\end{array}$} \\
\hline$B$ & $\beta$ & $\theta$ & & & & & & & & \\
\hline 60 & 0.0004 & 0.01 & 30 & 62.86 & 0 & 7388.18 & 40.61 & 10.56 & 30.82 & 18.01 \\
\hline 60 & 0.0005 & 0.01 & 35 & 64.44 & 0 & 8987.65 & 38.94 & 13.78 & 32.47 & 14.82 \\
\hline 60 & 0.0006 & 0.01 & 35 & 55.70 & 0 & 9656.32 & 36.25 & 13.55 & 36.55 & 13.66 \\
\hline 60 & 0.0008 & 0.01 & 41 & 69.89 & 0 & 10143.61 & 40.42 & 11.51 & 37.43 & 10.63 \\
\hline 60 & 0.001 & 0.01 & 48 & 37.91 & 0 & 11862.03 & 40.47 & 12.72 & 35.43 & 11.38 \\
\hline 60 & 0.001 & 0.02 & 48 & 40.00 & 0 & 11321.41 & 42.40 & 16.15 & 30.63 & 10.83 \\
\hline 60 & 0.001 & 0.1 & 48 & 49.59 & 0 & 14286.73 & 33.60 & 28.15 & 29.36 & 8.89 \\
\hline 60 & 0.001 & 0.5 & 48 & 88.72 & 0 & 18615.69 & 25.78 & 45.83 & 22.34 & 6.04 \\
\hline 60 & 0.001 & 1 & 30 & 129.82 & 0 & 21412.68 & 14.01 & 46.46 & 35.99 & 3.55 \\
\hline 60 & 0.002 & 0.04 & 69 & 33.67 & 0 & 16531.58 & 41.74 & 19.51 & 31.63 & 7.12 \\
\hline 120 & 0.0004 & 0.01 & 30 & 88.72 & 0 & 7311.29 & 41.03 & 14.85 & 37.39 & 6.73 \\
\hline 120 & 0.0005 & 0.01 & 34 & 65.28 & 0 & 8324.95 & 40.84 & 14.41 & 35.08 & 9.67 \\
\hline 120 & 0.0006 & 0.01 & 36 & 57.77 & 0 & 8821.24 & 40.81 & 14.05 & 36.61 & 8.52 \\
\hline 120 & 0.0008 & 0.01 & 41 & 146.77 & 0.49 & 10105.11 & 40.57 & 10.15 & 43.72 & 5.56 \\
\hline 120 & 0.001 & 0.01 & 47 & 129.36 & 0.05 & 11664.47 & 40.29 & 9.06 & 46.86 & 3.79 \\
\hline 120 & 0.001 & 0.02 & 48 & 102.74 & 0 & 11289.54 & 42.52 & 12.56 & 39.96 & 4.96 \\
\hline 120 & 0.001 & 0.1 & 47 & 125.74 & 0 & 14035.61 & 33.49 & 21.54 & 41.75 & 3.21 \\
\hline 120 & 0.001 & 0.5 & 47 & 204.66 & 0.57 & 18507.12 & 25.40 & 33.06 & 39.7 & 1.83 \\
\hline 120 & 0.001 & 1 & 29 & 109.30 & 0 & 20193.23 & 14.36 & 46.92 & 36.22 & 2.5 \\
\hline 120 & 0.002 & 0.04 & 69 & 37.70 & 0 & 16469.85 & 41.89 & 19.61 & 34.08 & 4.43 \\
\hline
\end{tabular}


Table 5.16.c. LP/NLP-BB computational performance for 150-node problems, $q=0.03, R=3$

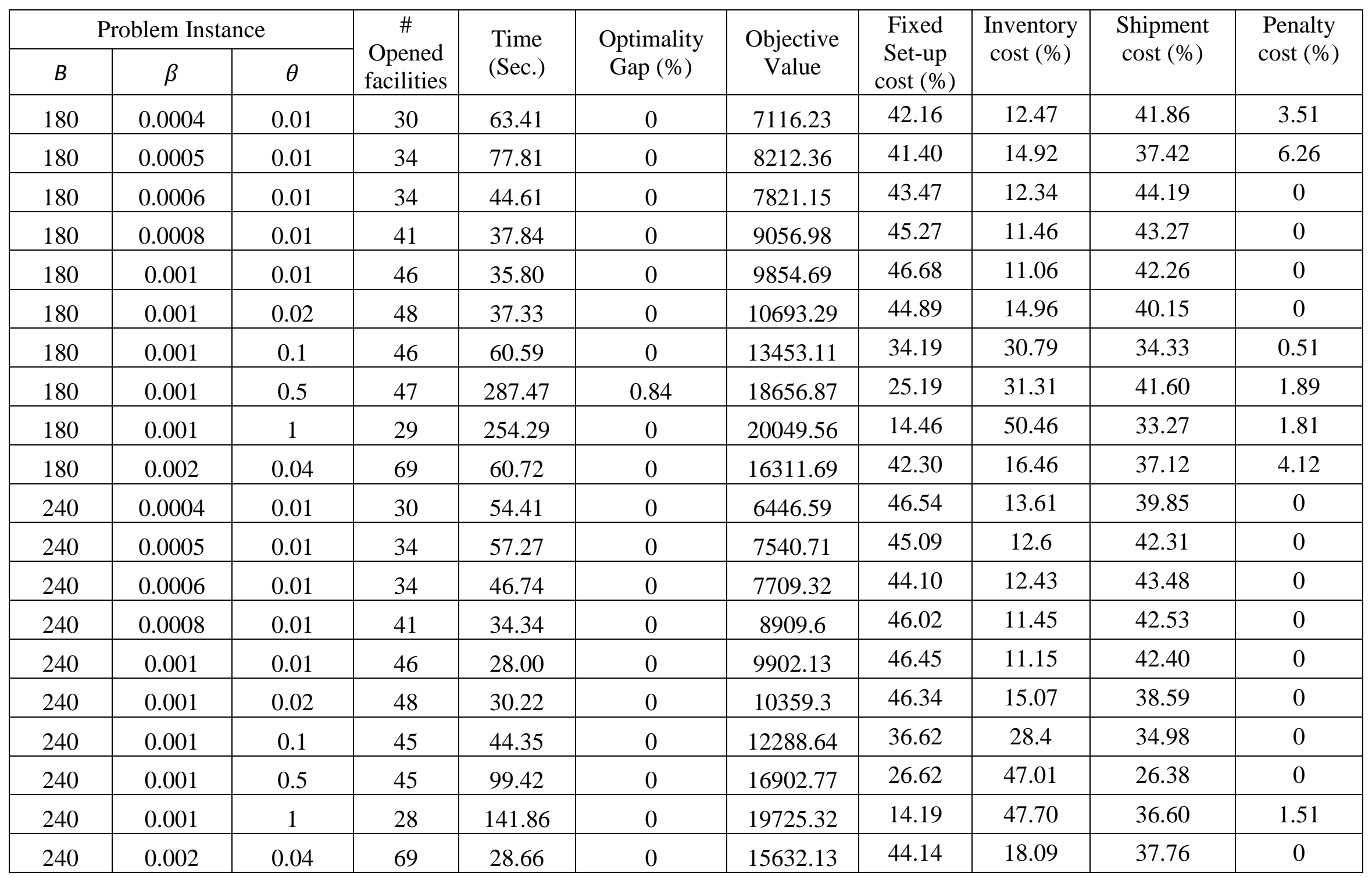


Table 5.16.d. LP/NLP-BB computational performance for 150-node problems, $q=0.03, R=3$

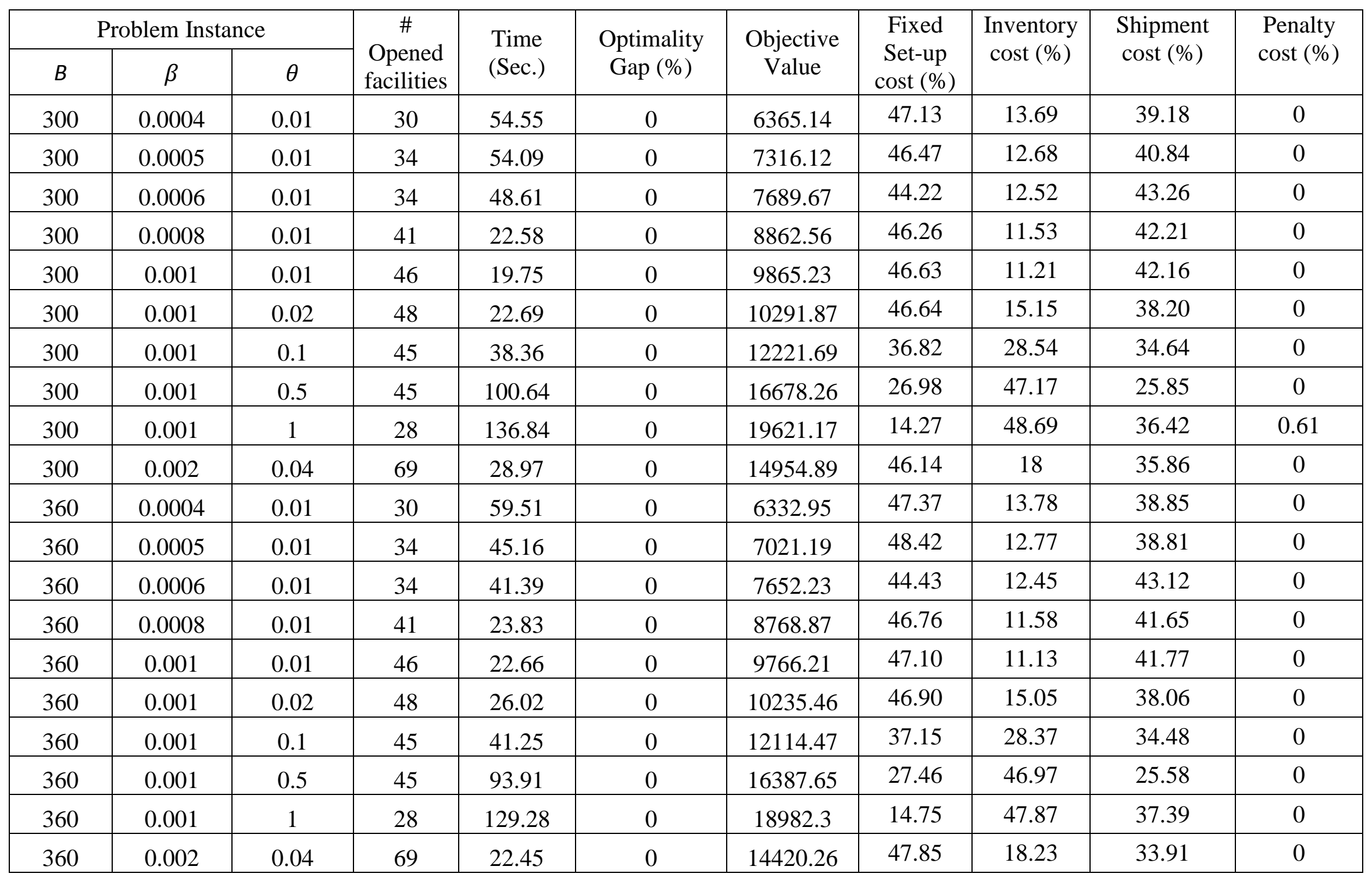


Table 5.17. Advantage of Planning for disruption: An example for 150-node problems, $q=0.001, R=3, B=180$

\begin{tabular}{|c|c|c|c|c|c|c|}
\hline \multicolumn{2}{|c|}{ Problem Instance } & \multirow{2}{*}{$\begin{array}{l}\text { Nominal } \\
\text { cost }\end{array}$} & \multirow{2}{*}{$\begin{array}{c}\text { Cost with no } \\
\text { planning }\end{array}$} & \multirow{2}{*}{$\begin{array}{l}\text { Cost with } \\
\text { planning }\end{array}$} & \multirow{2}{*}{$\begin{array}{c}\text { Increase (\%) } \\
\text { (No-planning) }\end{array}$} & \multirow{2}{*}{$\begin{array}{c}\text { Increase }(\%) \\
\text { (Planning) }\end{array}$} \\
\hline$\beta$ & $\theta$ & & & & & \\
\hline 0.0004 & 0.01 & 6223.72 & 21713.33 & 6409.94 & 248.88 & 2.99 \\
\hline 0.0005 & 0.01 & 6859.43 & 22348.63 & 7047.1 & 225.81 & 2.74 \\
\hline 0.0006 & 0.01 & 7481.41 & 22970.51 & 7669.95 & 207.03 & 2.52 \\
\hline 0.0008 & 0.01 & 8480.49 & 23968.91 & 8671.38 & 182.64 & 2.25 \\
\hline 0.001 & 0.01 & 9394.94 & 24883.4 & 9587.41 & 164.86 & 2.05 \\
\hline 0.001 & 0.02 & 9852.51 & 25340.73 & 10045 & 157.20 & 1.95 \\
\hline 0.001 & 0.1 & 11783.51 & 27270.78 & 11976.15 & 131.43 & 1.63 \\
\hline 0.001 & 0.5 & 16101.38 & 31586.49 & 16294.31 & 96.17 & 1.20 \\
\hline 0.001 & 1 & 18494.3 & 34820.33 & 18756.8 & 88.28 & 1.42 \\
\hline 0.002 & 0.04 & 13651.9 & 29138.64 & 13852.39 & 113.44 & 1.47 \\
\hline
\end{tabular}




\section{Chapter 6 - Conclusion}




\subsection{Summary}

Facility location decisions are the most critical decisions in designing efficient supply chains, as they cannot be changed once the facilities are built in the determined locations. While traditional facility location models, for simplicity, only take into account transportation and fixed facility setup costs, and assume that the facilities are non-failable, advances in computer technology over the past decades have made it possible to develop more challenging integrative models, which have motivated researchers to focus on improving the traditional models and creating models that work better in addressing real world problems. Recently, some researchers have concentrated on the incorporation of nonlinear inventory cost into the location modeling framework. The incorporation of inventory alters the optimal trade-off between transportation and facility setup costs, and significantly changes the location decisions. Another area (referred to as reliability location modeling) of research relaxes the non-failability assumption by taking into account that facilities are subject to disruption due to a variety of reasons such as natural disasters, fire incidents, power outages, transportation blockages, labor strikes, or terrorism. There are two types of remedy considered in the literature to protect against disruption: reactive strategy which suggests using backup facilities, and proactive strategy which considers a budget to fortify the facilities.

While higher inventory costs and higher facility setup costs result in the opening of fewer number of facilities, the consideration of facility disruption results in diversifying the disruption risk through opening up more facilities. Hence, higher risks of disruption, as well as higher transportation costs result in the opening of more facilities. That is why the integration of both inventory and disruption into the facility location modeling framework is a very important and interesting problem. However, integration studies are limited due to the difficulty and challenges 
that are presented by incorporation of the nonlinear inventory costs into the reliability location framework.

A reliable location-inventory model that considers both reactive (back-up facilities) and proactive (facility fortifications) remedial strategies was developed in this research. The developed model has two sources of non-linearity stemming from the inventory and fortification components of the model. First, the model was simplified and transformed into a more tractable problem, and then, providing a convexity proof for the nonlinear terms, two global search methods based on Outer Approximation (OA) and LP/NLP based branch and bound (LP/NLP-BB) solution approaches for mixed integer nonlinear program (MINLP), were developed to solve the problem. While the OA algorithm has a few straight steps, and is relatively simple to code, the LP/NLP-BB framework used in this research has some intermediate steps that use branch and bound features, such as primal heuristics and cutting management, which makes it more complex and much harder to code.

Three sets of computational experiments were designed to investigate the computational effectiveness of the developed methods and to derive managerial insights. The first set of experiments showed that the available commercial global solvers such as Branch-And-Reduce Optimization Navigator (BARON) are not capable of solving the problem, even for average-sized problems (e.g. 25-node). The second set of experiments, which included large-sized problems: 49node and 88-node, were considered to compare the performance of the developed methods. The results showed that the LP/NLP-BB framework significantly outperforms the OA approach, and provides more efficiently high quality solutions (within $0.5 \%$ of optimality). While the LP/NLPBB method provided the optimal solution (within 0.5\%) for all instances, the OA approach had difficulty solving the problem when the inventory cost is high. It also did not provide the optimal 
solution for many of the 88-node instances. Hence, only the LP/NLP-BB framework was considered for larger size problem (150 node) used in the third set of experiments. The results show that the solution approach based on LP/NLP-BB performs very efficiently for a broad range of parameters, and solves most of the instances to less than $1 \%$ optimality gap.

From a managerial viewpoint, as the chance of disruption $(q)$ increases, the number of opened facilities will increase which results in a higher total cost. Moreover, increasing the fortification budget $(B)$ results in more protection of the system, yielding lower number of opened facilities and total cost. Additionally, as expected, the number of opened facilities decreases whenever the inventory cost increases. However, the decrease in the number of opened facilities is not as significant as it normally is in other location inventory models in the literature (e.g. Daskin et. al., 2002). This is due to the risk-diversification effect, introduced by the reliability aspect of the problem. Lastly, a set of problem instances was used to show the efficiency of the model for making the system more resilient to disruptions. More specifically, it was shown that even when the chance of disruption is very low $(q=0.001)$, a dramatic increase (more than $200 \%)$ in the total cost due to facility failures can be prevented by slightly increasing the investments (up to $3 \%$ of the total cost) to plan for disruption.

\subsection{Future research}

The following areas may be considered for future studies:

- Consider the problem with uncertain demands for retailers, and include in the analysis safety stock for the opened facilities.

- While uncapacitated models are common for reliability location frameworks, it would be worthwhile to study and develop capacitated versions of the model 
presented in this research. Having nonlinear inventory costs as well as a capacitated reliability location framework would highly increase the complexity of the model. Developing an appropriate solution methodology would be a big challenge.

- Partial fortifications and heterogeneous facility disruptions are two other areas that can be considered to extend the location inventory model with risk of disruption, developed in this research. 


\section{REFERENCES}

Abhishek, K., Leyffer, S., and Linderoth, J. T. (2010). FilMINT: An outer-approximation-based solver for nonlinear mixed integer programs. INFORMS Journal on Computing, 22, 555-567.

Aboolian, R., Cui, T., \& Shen, Z. (2013). An Efficient Approach for Solving Reliable Facility Location Models. INFORMS JOURNAL ON COMPUTING, 25, 720-729.

Aksen, D., \& Piyade, N. (2010). The budget constrained r-interdiction median problem with capacity expansion. Central European journal of operations research, 18, 269-291.

Aryanezhad, M. B., Jalali, S. J., and Jabbarzadeh A. (2010). An integrated supply chain design model with random disruptions consideration. African Journal of Business Management, 4(12), 2393-2401.

Atamtürk, A., and Savelsbergh, M. (2005). Integer-programming software systems. Annals of Operations Research, $140,67-124$.

Atamtürk, A., Berenguer, G., \& Shen, Z. (2012). A Conic Integer Programming Approach to Stochastic Joint Location-Inventory Problems. Operations research, 60, 366-381.

Bailey, M. D., Shechter, S. M., \& Schaefer, A. J. (2006). SPAR: stochastic programming with adversarial recourse. Operations Research Letters, 34, 307-315.

Balinski, M. L. (1965). Integer Programming: Methods, Uses, Computation. Management Science, 12, 253-313.

Baumol, W. J., \& Wolfe, P. (1958). A Warehouse-Location Problem. Operations Research, 6, 252-263.

Beale, E.M.L. (1985). Integer programming. In: Sciitkowski, K. (ed.) Computational Mathematical Programming, NATO ASI Series, Springer, Berlin f15, 1-24.

Belotti, P., Kirches, C., Leyffer, S., Linderoth, J., Luedtke, J., and Mahajan, A., (2012). Mixed-Integer Nonlinear Optimization. Preprint ANL/MCS-P3060-1112.

Berman, O., \& Krass, D. (2011). On n -facility median problem with facilities subject to failure facing uniform demand. Discrete Applied Mathematics, 159, 420-432.

Berman, O., Krass, D., \& Menezes, M. B. C. (2007). Facility Reliability Issues in Network p-Median Problems: Strategic Centralization and Co-Location Effects. Operations research, 55, 332-350.

Berman, O., Krass, D. and Menezes, M. B. C. (2009). Locating facilities in the presence of disruptions and incomplete information. Decision Sciences, 40, 845-868.

Bonami, P., Biegler, L., Conn, A., Cornu’ejols, G., Grossmann, I., Laird, C., Lee, J., Lodi, A., Margot, F., Sawaya, N., and W"achter, A. (2008). An algorithmic framework for convex mixed integer nonlinear programs. Discrete Optimization, 5(2), 186-204.

Bundschuh, M., Klabjan, D. and Thurston, D. L. (2006), Modeling robust and reliable supply chains, Submitted manuscript, University of Illinois at Urbana-Champaign. 
Cappanera, P., \& Scaparra, M. P. (2011). Optimal Allocation of Protective Resources in Shortest-Path Networks. Transportation Science, 45, 64-80.

Chen, Q., Li, X., \& Ouyang, Y. (2011). Joint inventory-location problem under the risk of probabilistic facility disruptions. Transportation Research Part B, 45, 991-1003.

Church, R. L., Scaparra, M. P., \& Middleton, R. S. (2004). Identifying Critical Infrastructure: The Median and Covering Facility Interdiction Problems. Annals of the Association of American Geographers, 94, 491-502.

Church, R., \& Scaparra, M. P. (2007). Analysis of Facility Systems' Reliability When Subject to Attack or a Natural Disaster. in A. T. Murray and T. H. Grubesic, eds, 'Reliability and Vulnerability in Critical Infrastructure: A QuantitativeGeographic Perspective', Springer, chapter 11, pp. 221-241.

Church, R., \& Scaparra, M. (2007). Protecting critical assets: The r-interdiction median problem with fortification. Geographical Analysis, 39, 129-146.

Cui, T., Ouyang, Y., \& Shen, Z. M. (2010). Reliable Facility Location Design Under the Risk of Disruptions. Operations research, 58, 998-1011.

Daganzo, C. F., \& Newell, G. F. (1986). CONFIGURATION OF PHYSICAL DISTRIBUTION NETWORKS. Networks, 16, 113-132.

Dakin, R. J. (1965). A tree search algorithm for mixed programming problems. Computer Journal, 8, 250-255.

Danna, E., Rothberg, E., and LePape, C. (2005). Exploring relaxation induced neighborhoods to improve MIP solutions. Mathematical Programming, 102, 71-90.

Daskin, M.S. (1995). Network and Discrete Location: Models, Algorithms, and Applications. Wiley, New York.

Daskin, M.S. (2013). Network and Discrete Location: Models, Algorithms, and Applications. Wiley, New York.

Daskin, M. S., Coullard, C. R., \& Shen, Z. M. (2002). An Inventory-Location Model: Formulation, Solution Algorithm and Computational Results. Annals of Operations Research, 110, 83-106.

Drezner, Z. (1987). Heuristic Solution Methods for Two Location Problems with Unreliable Facilities. The Journal of the Operational Research Society, 38, 509-514.

Duran, M. A. and Grossmann, I. (1986). An outer-approximation algorithm for a class of mixed integer nonlinear programs. Mathematical Programming, 36, 307-339.

Forrest, J. J. H., Hirst, J. P. H. and Tomlin J. A. (1974). Practical solution of large scale mixed integer programming problems with UMPIRE. Management Science, 20, 736-773.

Fletcher, R. and Leyffer, S. (1994). Solving mixed integer nonlinear programs by outer approximation. Mathematical Programming, 66, 327-349.

Fulkerson, D. R., \& Harding, G. C. (1977). Maximizing the minimum source-sink path subject to a budget constraint. Mathematical Programming, 13, 116-118.

Garcia-Herreros P, Wassick JM, Grossmannn IE. (2014) Design of resilient supply chains with risk of facility disruptions. Industrial \& Engineering Chemistry Research,53(44), 17240-51. 
Geoffrion, A. M. (1972). Generalized Benders decomposition. Journal of Optimization Theory and Applications, 10(4), 237-260.

Golany, B., Kaplan, E. H., Marmur, A., \& Rothblum, U. G. (2009). Nature plays with dice - terrorists do not: Allocating resources to counter strategic versus probabilistic risks. European Journal of Operational Research, 192, 198-208.

Golden, B. (1978), ‘A problem in network interdiction’, Naval Research Logistics Quarterly 25, 711-713.

Grossmann, I. E. (2002). Review of nonlinear mixed-integer and disjunctive programming techniques. Optimization and Engineering, 3, 227-252.

Grossmann, I. E. and Kravanja, Z. (1997). Mixed-integer nonlinear programming: A survey of algorithms and applications. In L.T. Biegler, T.F. Coleman, A. C. and Santosa, F., editors, Large- Scale Optimization with Applications, Part II: Optimal Design and Control, Springer, New York.

Gupta, O. K. and Ravindran, A. (1985). Branch and bound experiments in convex nonlinear integer programming. Management Science, 31, 1533-1546.

Hakimi, S. L. (1964). Optimum Locations of Switching Centers and the Absolute Centers and Medians of a Graph. Operations research, 12, 450-459.

Jabbarzadeh, A., Jalali Naini, S.G., Davoudpour, H., Azad, N., (2012). Designing a supply chain network under the risk of disruptions. Mathematical Problems in Engineering. http://dx.doi.org/10.1155/2012/234324

Janjarassuk, U., \& Linderoth, J. (2008). Reformulation and sampling to solve a stochastic network interdiction problem. Networks, 52, 120-132.

Johnson, E. L., Nemhauser, G. L. and Savelsbergh, M. W. P. (2000). Progress in linear programming based branchandbound algorithms: Exposition. INFORMS Journal of Computing, 12.

Kleywegt, A., Shapiro, A., \& Homem-De-Mello, T. (2001). The sample average approximation method for stochastic discrete optimization. SIAM JOURNAL ON OPTIMIZATION, 12, 479-502.

Kouvelis, P., Kurawarwala, A., \& Gutierrez, G. (1992). Algorithms for robust single and multiple period layout planning for manufacturing systems. European Journal of Operational Research, 63, 287-303.

Krass, D., \& Berman, O. (2009). Locating facilities in the presence of disruptions and incomplete information. Decision sciences, 40, 845-868.

Lee, S. (2001). On solving unreliable planar location problems. Computers and Operations Research, 28, 329-344.

Leyffer, S. (2001). Integrating SQP and branch-and-bound for mixed integer nonlinear programming. Computational Optimization \& Applications, 18, 295-309.

Li, Q. (2011). Decision Support Models for Design of Fortified Distribution Networks. Ph.D. Thesis. University of South Florida.

Li, Q., Zeng, B., \& Savachkin, A. (2013). Reliable facility location design under disruptions. Computers and Operations Research, 40, 901-909 
Li, X., \& Ouyang, Y. (2010). A continuum approximation approach to reliable facility location design under correlated probabilistic disruptions. Transportation Research Part B, 44, 535-548.

Liberatore, F., \& Scaparra, M. P. \& Daskin, M. S. (2011). Optimizing Protection Strategies for Supply Chains: Comparing Classic Decision-Making Criteria in an Uncertain Environment. Annals of the Association of American Geographers, 101, 1241-1258.

Liberatore, F., Scaparra, M. P. and Daskin, M. S. (2012), Optimization methods for hedging against disruptions with ripple effects in location analysis, Omega 40, 21-30.

Linderoth, J. T. and Savelsbergh, M. W. P. (1999). A computational study of search strategies in mixed integer programming. INFORMS Journal on Computing, 11, 173-187.

Lim, M., Daskin, M., Bassamboo, A., \& Chopra, S. (2010). A Facility Reliability Problem: Formulation, Properties, and Algorithm. NAVAL RESEARCH LOGISTICS, 57, 58-70.

Lim, M. K., Bassamboo, A., Chopra, S., \& Daskin, M. S. (2013). Facility location decisions with random disruptions and imperfect estimation. Manufacturing and Service Operations Management, 15, 239-249.

Losada, C., Scaparra, M. P., Church, R. L., \& Daskin, M. S. (2012). The stochastic interdiction median problem with disruption intensity levels. Annals of Operations Research, 201, 345-365.

Losada, C., Scaparra, M. P. \& Church, R. L. (2010a), Interdiction of p-median systems with facility recovery time and frequent disruptions: A resiliency analysis. Working paper, Kent Business School, University of Kent, UK.

Losada, C., Scaparra, M. P., \& Church, R. L. (2010b). On a bi-level formulation to protect uncapacitated p-median systems with facility recovery time and frequent disruptions. Electronic Notes in Discrete Mathematics, 36, 591-598.

Miranda, P. A., \& Garrido, R. A. (2006). A Simultaneous Inventory Control and Facility Location Model with Stochastic Capacity Constraints. Networks and Spatial Economics, 6, 39-53.

Miranda, P. A., \& Garrido, R. A. (2004). Incorporating inventory control decisions into a strategic distribution network design model with stochastic demand. Transportation Research Part E, 40, 183-207.

Nabar S. \& Schrage, L. (1991). Modeling and solving nonlinear integer programming problems, Presented at Annual AIChE Meeting, Chicago.

O'Hanley, J. R. and Church, R. L. (2011), Designing robust coverage networks to hedge against worst-case facility losses, European Journal of Operational Research 209, 23-36.

O'Hanley, J. R., Church, R. L., \& Gilless, J. K. (2007a). The importance of in situ site loss in nature reserve selection: Balancing notions of complementarity and robustness. Biological Conservation, 135, 170-180.

O’Hanley, J. R., Church, R. L., \& Gilless, J. k. (2007b). Locating and protecting critical reserve sites to minimize expected and worst-case losses. Biological Conservation, 134, 130-141.

Owen, S. H., \& Daskin, M. S. (1998). Strategic facility location: A review. European Journal of Operational Research, 111, 423-447.

Ozsen, L., Coullard, C. R., \& Daskin, M. S. (2008). Capacitated warehouse location model with risk pooling. Naval Research Logistics (NRL), 55, 295-312. 
Peng, P., Snyder, L. V., Lim, A., \& Liu, Z. (2011). Reliable logistics networks design with facility disruptions. Transportation Research Part B, 45, 1190-1211.

Qi, L., \& Shen, Z. M. (2010). The effect of supply disruptions on supply chain design decisions. Transportation science, 44, 274-289.

Qi, L., \& Shen, Z. M. (2007). A supply chain design model with unreliable supply. Naval Research Logistics (NRL), $54,829-844$.

Quesada, I. and Grossmann, I. E. (1992). An LP/NLP based branch-and-bound algorithm for convex MINLP optimization problems. Computers \& Chemical Engineering, 16, 937-947.

Romeijn, H. E., Shu, J., \& Teo, C. (2007). Designing two-echelon supply networks. European Journal of Operational Research, 178, 449-462.

Scaparra, M. P., \& Church, R. L. (2008a). A bilevel mixed-integer program for critical infrastructure protection planning. Computers and Operations Research, 35, 1905-1923.

Scaparra, M. P., \& Church, R. L. (2008b). An exact solution approach for the interdiction median problem with fortification. European Journal of Operational Research, 189, 76-92.

Scaparra, M. P., \& Church, R. (2012). Protecting supply systems to mitigate potential disaster: a model to fortify capacitated facilities. International regional science review, 35, 188-210.

Scaparra, M. P., \& Liberatore, F. (2011). Analysis of facility protection strategies against an uncertain number of attacks: the stochastic R-interdiction median problem with fortification. Computers \& Operations Research, 38, 357 366.

Shahabi, M., Unnikrishnan, A., Jafari-Shirazi, E., Boyles, S. D. (2014). A three level location-inventory problem with correlated demand. Transportation Research Part B, 69, 1-18.

Shen, Z., Zhan, R., \& Zhang, J. (2011). The Reliable Facility Location Problem: Formulations, Heuristics, and Approximation Algorithms. INFORMS JOURNAL ON COMPUTING, 23, 470-482.

Shen, Z. M., Coullard, C., \& Daskin, M. S. (2003). A Joint Location-Inventory Model. Transportation Science, 37, 40-55.

Sherali, H. D., \& Alameddine, A. (1992). A new reformulation-linearization technique for bilinear programming problems. Journal of Global Optimization, 2, 379-410.

Shu, J., Sun, J. (2006). Designing the distribution network for an integrated supply chain. Journal of Industrial and Management Optimization, 2, 339-349.

Shu, J., Teo, C., \& Shen, Z. M. (2005). Stochastic Transportation-Inventory Network Design Problem. Operations research, 53, 48-60.

Snyder, L. V. (2006). Facility location under uncertainty: A review. IIE Transactions (Institute of Industrial Engineers), 38, 547-564.

Snyder, L. V., \& Daskin, M. S. (2007). Models for Reliable Supply Chain Network Design. in A. T. Murray and T. H. Grubesic, eds, 'Reliability and Vulnerability in Critical Infrastructure: A Quantitative- Geographic Perspective', Springer, chapter 13, pp. 257-289. 
Snyder, L. V., \& Daskin, M. S. (2005). Reliability Models for Facility Location: The Expected Failure Cost Case. Transportation Science, 39, 400-416.

Snyder, L. V., Daskin, M. S., \& Teo, C. (2007). The stochastic location model with risk pooling. European Journal of Operational Research, 179, 1221-1238.

Snyder L. V., Scaparra M. P., Daskin, M. S. and Church R. L. (2006). Planning for disruptions in supply chain networks, Tutorials Oper Res, INFORMS, 234-257.

Snyder L. V., Zümbül A, Peng P, Rong Y, Schmitt A, Sinsoysal B (2012). OR/MS models for supply chain disruptions: A review. Working paper, Lehigh University, Bethlehem, PA

Savelsbergh, M. W. P. (1994). Preprocessing and probing techniques for mixed integer programming problems. ORSA Journal on Computing, 6, 445-454.

Sourirajan, K., Ozsen, L., Uzsoy, R. (2007). A single-product network design model with lead time and safety stock considerations. IIE Transactions 39, 411-24.

Sourirajan, K., Ozsen, L., \& Uzsoy, R. (2009). A genetic algorithm for a single product network design model with lead time and safety stock considerations. European Journal of Operational Research, 197, 599-608.

Stubbs, R. and Mehrotra, S. (1999). A branch-and-cut method for 0-1 mixed convex programming. Mathematical Programming, 86, 515-532.

Teo, C., \& Shu, J. (2004). Warehouse-Retailer Network Design Problem. Operations research, 52, $396-408$.

Viswanathan, J. and Grossmann, I. E. (1990). A combined penalty function and outer approximation method for MINLP optimization. Computers \& Chemical Engineering, 14(7), 769-782.

Westerlund, T. and Pettersson, F. (1995). A cutting plane method for solving convex MINLP problems. Computers \& Chemical Engineering, 19, s131-s136.

Wollmer, R. (1964). Removing Arcs from a Network. Operations research, 12, 934-940. 